\title{
RESEARCH AND DEVELOPMENT OF METHODS AND TOOLS FOR ACHIEVING AND MAINTAINING CONSENSUS PROCESSES IN THE FACE OF CHANGE WITHIN AND AMONG GOVERNMENT OVERSIGHT AGENCIES
}

\author{
Volume I \\ Progress Report \\ for Period October 1, 1991 - September 30, 1992 \\ Management Systems Laboratories \\ Virginia Polytechnic Institute and State University \\ Blacksburg, Virginia 24060
}

March 1992

Prepared for

The U. S. Department of Energy

Agreement No. DE-FG02-88DP48058 


\section{NOTICE}

This report was prepared as an account of work sponsored by the United States Government. Neither the United States nor the Department of Energy, nor any of their employees, nor any of their contractors, subcontractors, or their employees, makes any warranty, expressed or implied, or assumes any legal liability or responsibility for the accuracy, completeness, or usefulness of any information, apparatus, product or process disclosed or represents that its use would not infringe privatelyowned rights. 
This progress report summarizes our research activities under our consensus grant. In year four of the grant, we continued to capitalize on and benefit from historical events which drove our early emphasis on group process studies. Following our work on various procedures for bringing together groups such as the State and Tribal Government Working Group and the Stakeholders' Forum (both of which provide input to the Five-Year Waste Plan), we continue to observe these groups and collect data. We also began a configuration study involving the complex modeling of DOE's Office of Environmental Restoration and Waste Management (EM). Related to group process studies is the issue of the information requirements for individuals making decisions in consensus groups. Our information studies examined the requirements for decision-related information, frameworks for such information, and the effectiveness of information portrayed for decision making. However, we were able not only to continue studying consensus groups in action and related information issues, but also to focus considerable attention on the fundamental side of our research. The fundamental or basic research conducted in year four included: 1) expanding our literature database; 2) beginning the writing of the literature review summary document and the consensus guide; 3) developing frameworks and models such as the Environmental Trilogy model and a structural equations model of the consensus process; and 4) conducting laboratory studies concerning the effects of the presence of an expert, met expectations, opportunity to express views, incentive structure and conflict type (competitive versus collaborative) on consensus outcomes.

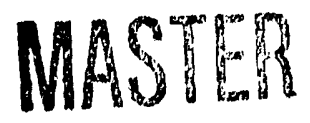


TABLE OF CO YTENTS

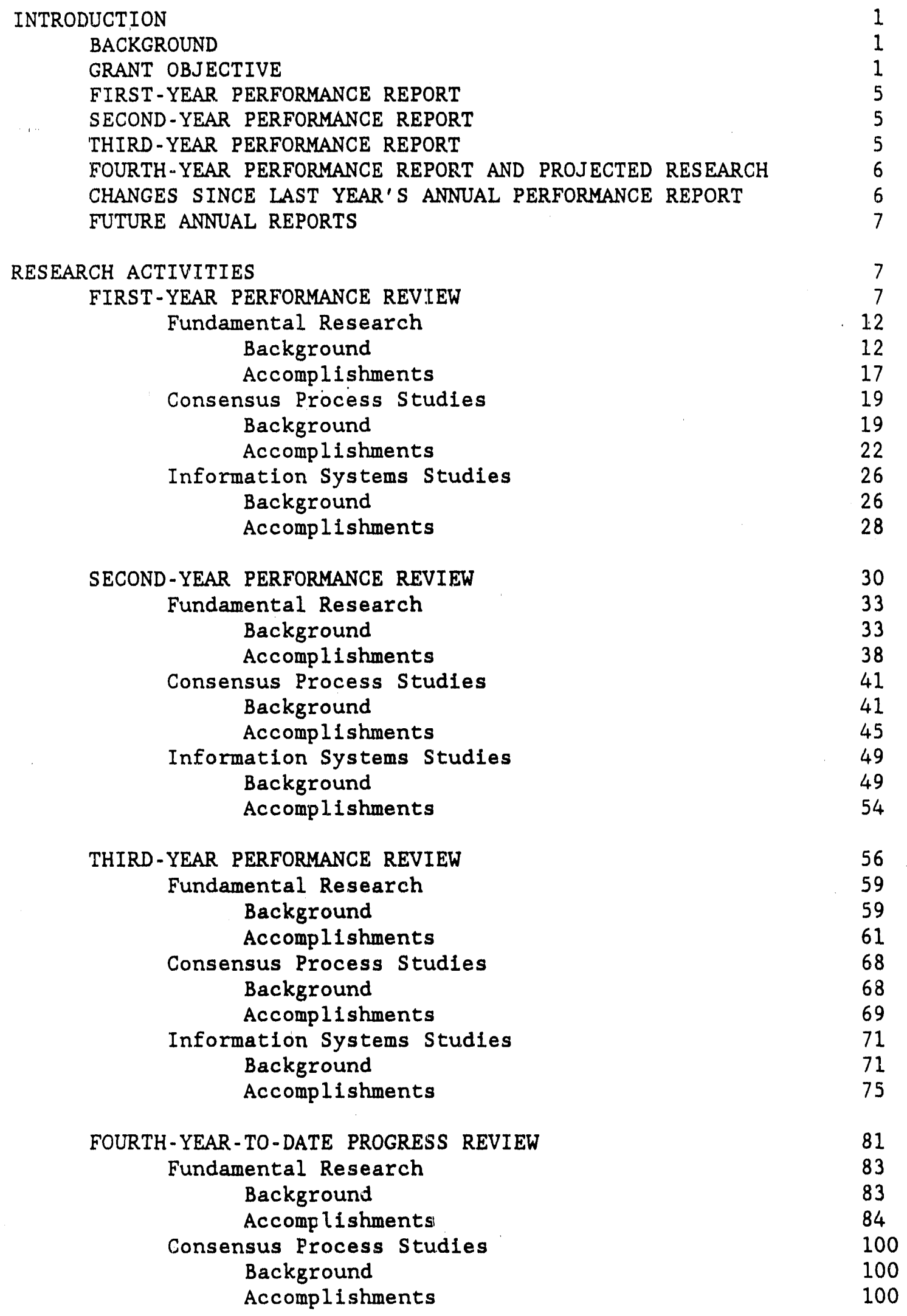


STUDY RESULTS: MILESTONE LISTINGS 110 LISTING OF GRANT STUDIES

FIRST YEAR STUDY RESULTS: Key to Figure 9

$\begin{array}{ll}\text { GRANT MANAGEMENT STRUCTURE } & 135\end{array}$

EQUIPMENT

First Year 
TABLE OF CONTENTS (con't)

\section{List of Figures}

Figure 1. The Research Concept (showing the inward path) proposes six effort areas leading to the research objectives......... 3

Figure 2. The research plan establishe: annual objectives for each

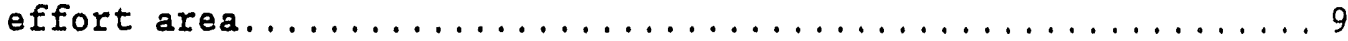

Figure 3. Consensus can be pictured as a continuum.............. 15

Figure 4. There are certain factors required for supporting

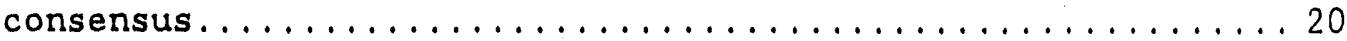

Figure 5. Operational Criteria are mat hed to different endeavors.....53

Figure 6. Our paradigm illustrates consensus, the variables affecting $i t$, and the interrelationships of the variables............................... 60

Figure 7. The data and information characteristics framework.......76

Figure 8. This structural model portrays the hypothesized relationship between type of expertise, personality dispositions of dominance and affiliation, and consensus variables............................... 94

Figure 9. This figure shows our progress toward accomplishing research objectives within each domain during the

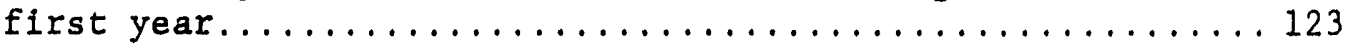

Figure 10 This figure shows our progress toward accomplishing research objectives within each domain during the

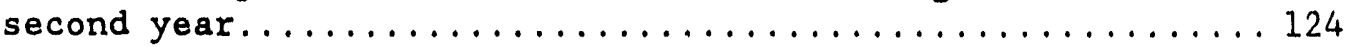

Figure 11. This figure shows our progress toward accomplishing research objectives within each domain during the third ytar................................... 125

Figure 12. This figure shows our progress toward accomplishing research objectives within each domain during the fourth year. 
TABLE OF CONTENTS (con't)

Appendix: Graduate Students Performing Consensus Grant Research for Advanced Degrees 
INTRODUCTION

BACKGROUND

On September 30, 1988, the Office Director of Defense Waste and Transportation Management entered into a Research Grant on Consensus with Management Systems Laboratories (MSL) of Virginia Polytechnic Institute and State University (Virginia Tech). Dr. Harold A. Kurstedt, Jr., the Hal G. Prillaman Professor of Industrial and Systems

Engineering and Professor of Nuclear Engineering, and MSL's director, is the principal investigator. The grant is based on a proposal entitled, "Research and Development of Methods and Tools for Achieving and Maintaining Consensus Processes in the Face of Change Within and Among Government Oversight Agencies (June 1988)."

MSL is a functional part of the Department of Industrial and Systems Engineering within the College of Engineering at Virginia Tech.

\section{GRANT OBJECTIVE}

In contrast to the autonomy of authoritarian rule implemented through edict and backed by force, the key defining feature of the organizations responsible for making American government work today is "polyonomy," a term we've invented to signify that responsibility and power are lodged not with any one agency but are apportioned among many agencies and subagencies. Polyonomy requires consensus backed by negotiation and persuasion. Government Oversight Agencies (GOA's)--those bodies at all levels of government (federal, state, and local) charged with implementing laws made by the Congress and by state legislatures--face the monumental but essential task of coordinating their overlapping roles and responsibilities. And that's not all. They must learn to cooperate within an environment which engenders and rewards dissenting views, and they must further learn to maintain the ability to cooperate over long periods of time, to coordinate necessary adaptation to inevitable change. The need is urgent for longitudinal, real-world research on mechanisms to create and maintain processes for helping GOA's achieve quality consensus decisions.

We hypothesize that consensus evolves and recycles through four phases-Planning, Building/Maintaining, Implementing, and Post-Implementation-within an external environment determined by four elements--Compulsions (Drivers), Information Gathering, Communications, and Reward Systems. Consensus can be strong or fragile, real or apparent, healthy or unhealthy. Our aim as researchers is not to judge whether a particular consensus decision is good, bad, or neither good nor bad; but rather to understand consensus and the kinds of consensus, including the factors contributing to these kinds and the necessary steps to take in achieving the kind of consensus desired.

To move GOA's toward successful implementation of high-quality decisions involving large and serious national programs and requiring the participation and consensus of many agencies, jurisdictions, and 
interest groups both within and outside the primary responsible GOA's direct domain, MSL, under the grint, plans to accomplish the following objectives:

To use the real-world laboratory of the U.S. Department of Energy (DOE) Office of Defense Waste and Transportation Management (DWTM) Environmental Restoration Program (ERP) as a generic GOA test case to understand consensus: its definition, characterization, measurement, implementation, improvement, management, and effects on other things;

To find, study, invent, develop, and refine better tools and methods for consensus, integrating them in a tested consensus process or processes, and documenting the integrated results in a Guide for GOA managers;

To determine the ways and degrees participative processes are of value to GOA's; and

To improve decision-making within and among GOA's by giving them the methods and tools to achieve and maintain consensus processes in the face of change.

Our proposed research focuses on participative, consensus-based decision processes and on building into these processes the flexibility needed to participatively adapt consensus-based decisions in the face of inevitable changes. Our approach to meeting our research objective is to conduct research and development in six interrelated effort areas: theory and concepts, test sites, consensus tools, frameworks and models, consensus methods, and consensus process. Figure 1 represents our Research Concept, which forms the basis for our technical approach and our research plan. Our activities in the six effort areas relate to each other, as we take the clockwise and inward path of exploration indicated by the figure. The work will also advance the body of knowledge relating to consensus, as, moving counterclockwise and outward, we discover, refine, and confirm theory and concepts. This dual-path approach allows us to achleve the overall goal of the proposed research: both a theoretical and an applied understanding of consensus. 


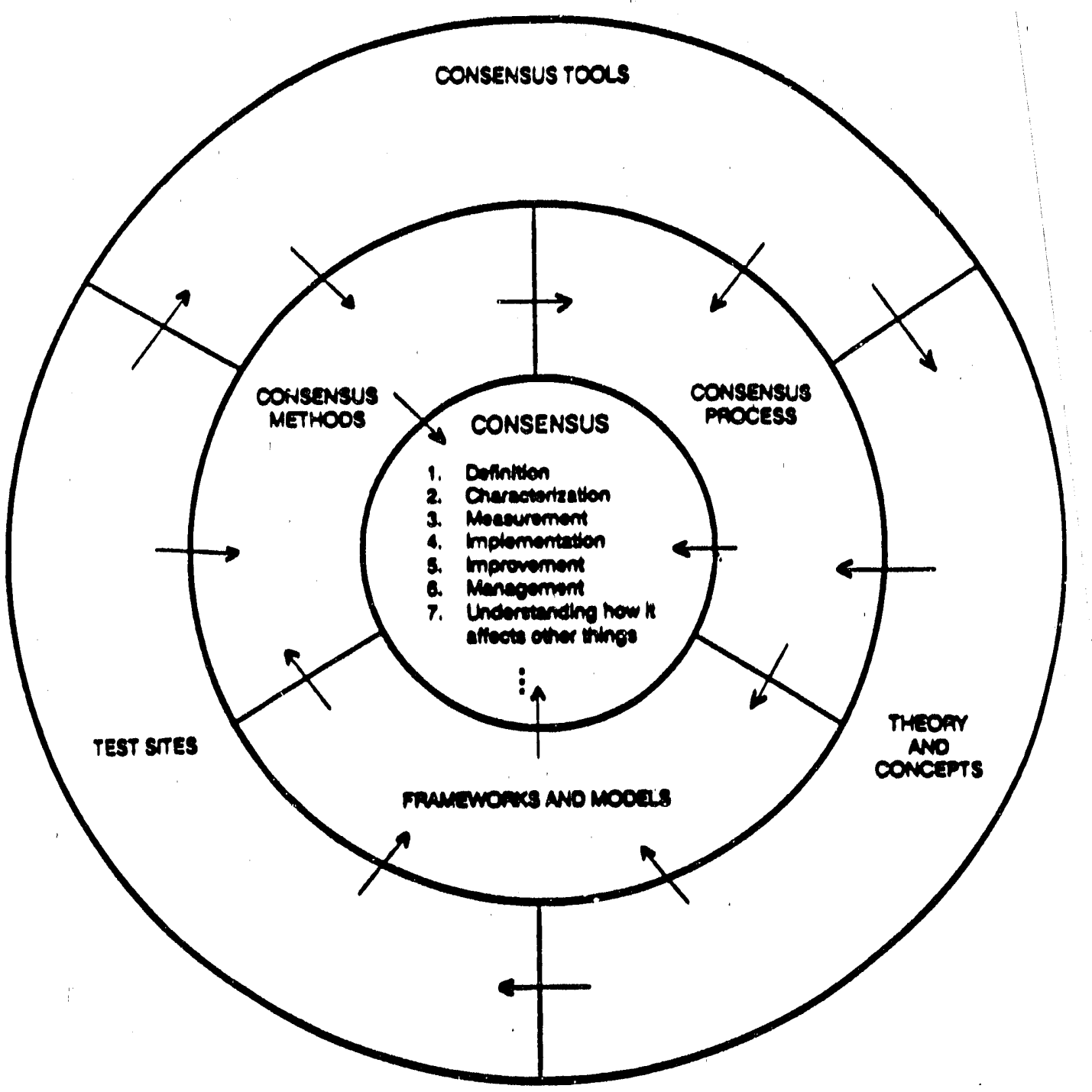

Figure 1. The Research Concept (showing the inward path) proposes six effort areas leading to the research objectives.

We distinguish between Consensus Methods and Consensus Process, as used in Figure 1, in the following way. "The word method, thought to have first appeared in Plato's Phaedrus, comes from two Greek roots mearing along and way. Method therefore refers to the serles of steps taken along the way to a given end. It tells what you must do to achieve a specific goal" (Gould et al., 1988). We view a process as the application of a set of methods linked by rules. The distinction we make between a method and a process is that the relationships between the steps in a method are well defined, whereas the relationships between the steps (or groups of steps called methods) in a process are not necessarily well defined. The latter relationships have a degree of uncertainty. The rules in the process are heuristic, offering the manager expert advice on assessing which steps to execute at any point-"Before you do this, be sure to take that and the other into account," or, "If such-and-such happens, it looks like you could do one or both of the following things." Rules are probabilistic, highly-educated guesses, as contrasted with the linkage in the method, which is certain- If you do this and then this, you'11 get what you need." 
If we look at the Research Concept and the relationship arrows in Figure 1 , we can get an overall picture of how thi pieces fit. To understand consensus, we need to explore things about consensus. We need to define it, characterize it, measure it, implemert it, improve it, manage it, and understand how it affects other things.

In Figure 1, the dots in the center circle represent our realization that there may well be other factors involved in understanding consensus, which will come to our attention as we move forward from our current knowledge and experience.

The question is, how do we go about this comprehensive and monumental task? Let's look at Figure 1. Frameworks and Models represent our hypotheses (our current understanding) about consensus. These hypotheses are grounded in established Theory and our experience with the Test Sites. From these frameworks, we see the need for Consensus Methods. Some of these methods exist; most existing methods, we believe, need changes; and many methods--especially in the area of measuring consensus (both kind and degree; both during group decisionmaking and later on)--will have to be developed. Methods require Tools to implement and sites for testing to ensure the mothods do what we expect. The next logical question is how to combine these specific methods to obtain consensus. Rules connect methods and indicate tools and tool preferences. We therefore hypothesize about the Consensus Process and perform tests to see if the tools, methods, and rules we've developed accomplish a higher degree of non-fragile consensus. This is what we call the forward (clockwise and inward) pass through the Research Concept.

Once we've reached a new understanding consensus through the forward pass, we take the backward (counterclockwise and outward) pass through the steps, which results in verification or revision of the Theory and Concepts surrounding consensus. The two passes are analogous to a mathematical proof, which starts with the theory and executes to some point of finality (Forward Pass), then hits the "Therefore" statement to prove false or some aspect of the theory (Backward Pass).

Though we've explained the relationships in the concept in a sequential (serial) manner for clarity, they are often executed simultaneously (or in parallel). The flow of results is most often understood logically in a clockwise, outside-to-inside manner starting with Theory and Concepts and ending with the Consensus Process.

We'11 derive new theories, concepts, frameworks, and models for consensus and confirm (or confirm modifications of) existing ones. From these we'll derive well-defined tested methods comprising tools for generating, measuring, and managing consensus. Benefits will include more and better participatively-generated ideas and decisions on prioritization of program activities; constructive, not destructive, involvement of legitimate interests through a sense of mutual ownership and cooperative concern; sustained program momentum through decreasing intra- and inter-organizational conflicts-in short, maximizing the amount of program energy devoted to program tasks and minimizing the 
amount of energy wasted on frustration and friction among polyonomous program elements.

Everything we do in our proposed research will in some way culminute in our envisioned Guide to the Consensus Process. This Guide, an expert system for managing consensus processes, designed to replicate the behavior of a deeply knowledgeable and practiced GOA consensus consultant, will show how to apply the right sets of methods according to the right sets of rules to achieve and maintain consensus decisions within the total variety of program-managerial situations. The Guide will be a major (and unprecedented) consensus management and training tool, portending more lasting and more predictable program success than could otherwise be hoped for. In addition, as the research progresses, the idea of this evolving Guide will. serve a focusing, vectoring role analogous to that of the integrative study of the late 1970's at Savannah River within the Alternate Fuel-Cycle Technology Program. This expert system is an excellent candidate for development as first a document and later an automated tool, the development of both of which.including the many sub-efforts on which their construction depends--will furnish fertile ground for Masters theses and Doctoral dissertations.

\section{FIRST-YEAR PERFORMANCE REPORT}

Our annual performance report describes the previous year's research efforts. To meet the May 29, 1989 deadline, the reported content in our First Annual Performance Report ended May 1, 1989. Since our grant started on September 30,1988, our performance and reporting years overlap by five months.

\section{SECOND-YEAR PERFORMANCE REPORT}

In our Second Annual Performance Report, we describe our performance for the first seven months of the second grant year, covering the period September 30, 1989 through May 1, 1990. We'll review this partial second-year performance against the second-year projections in our proposal and the profections in last year's annual performance report. We also updated the remaining months of the first grant year not covered in the First Year Performance report. This report will review the partial second grant year under the heading, "Grant Second-Year-to-Date Progress Review."

\section{THIRD YEAR PERFORMANCE REPORT}

In our Third Annual Performance Report, we describe our performance for the first five months of the third grant year, covering the period September 30,1990 - March 1, 1991. We'll review this partial thirdyear performance against the third-year projections in our proposal and the projections in last year's annual performance report. This report will review the partial third grant year under the heading "Grant ThirdYear-to-Date Progress Review." We updated the Grant Second-Year-to-Date Progress Review with the remaining five months of grant year two. Since 
this report is cumulative, the Third Annual Performance Report includes all of grant year one and two, plus the first five months of grant year three.

\section{FOURTH YEAR PERFORMANCE REPORT AND PROJECTED RESEARCH}

In our Fourth Annual Performance Report, we describe our performance for the first five months of the fourth grant year, covering the period September 30, 1991 - March 1, 1992. We'11 review this partial fourthyear performance against the fourth-year projections in our proposal and the profections in last year's annual performance report. This report will review the partial fourth grant year under the heading "Grant Fourth-Year-to-Date Progress Review." We updated the Grant Third-Yearto-Date Progress Review with the remaining seven months of grant year three. Since this report is cumulative, the Fourth Annual Performance Report hacludes all of grant year one, two, and three plus the first five months of grant year four.

\section{CHANGES SINCE LAST YEAR'S ANNUAL PERFORMANCE REPORT}

Increased emphasis on modelling the EM environment is reflected in our Grant Fourth-Year-to-Date Progress Review, and our continuation proposal for year five.

During the first two years, we spent the majority of our efforts under the domains of consensus processes and information systems studies. We established our relationships with the managers and staff of DOE's Office of Environmental Restoration and Waste Management (EM), formed consensus groups beneficial to EM's programmatic mission to create opportunities for studying consensus, and we worked in areas of EM to gain an understanding of the organization, its processes, and the programmatic mission. These are prerequisites for studying consensus in the real-world environment. These studies focused on understanding the body of knowledge--typically limited to a literature review in basic research and establishing the research environment. We accomplished the above objectives through assisting with EM's five-year planning, organizing and facilitating various groups such as the States and Tribal Government Working Group (STGWG), and establishing and running the EM task force on information systems studies.

Early in year three, we made a concerted effort to identify those activities which have matured and should be moved to EM's organization. We have also identified studies completed through different contractual vehicles and/or organizations. Therefore, a number of studies have been completed under this grant.

The work under the domains of consensus processes and information systems studies will now focus on developing models of the real-world environment related to the conceptual models of consensus developed in the domain of fundamental research. These models coupled with the results of fundamental research on consensus should enable us to develop 
instruments for measuring consensus in the real-world, design real-world studies, and develop studies realistic to EM.

FUTURE ANNUAL REPORTS

Due to the tight integration of the many studies of the grant over the six research phases (effort areas) throughout the five grant years, we intend for each annual performance report to be comprehensive. Each report will reproduce prior completed years, complete the partial report of the most recent part-year, and report on the current part-year. The fifth and final report is due 90 days after completion of the grant year. Thus, next year's report, the fifth and final, will include sections on Grant First-Year Performance Review, Grant Second-Year Performance Review, Grant Third-Year Performance Review, Grant FourthYear Performance Review, and Grant Fifth-Year Performance Review.

\section{RESEARCH ACTIVITIES}

\section{FIRST-YEAR PERFORMANCE REVIEW}

We submitted our proposal and received rur grant on consensus before the election of 1988. And because of the election of 1988 resulting in the election of George Bush and his appointment of Admiral Watkins, the Department of Energy is committed to changing its culture based on a consensus orientation. What a fortuitous and unexpected course of events for our research efforts! Our life, instead of trying to promote some consensus activities and find areas and set up test sites where we might study consensus, has become one where our whole researchlaboratory world (EM) has turned into large and comprehensive consensus activities. Test sites are everywhere. The opportunities for consensus studies are enormous. We've been thrown right into the middle of it. Data for studying consensus are everywhere and our job is to organize our observations and define consensu: variables so we can take advantage of the opportunity.

Consequently, much of our first grant year dealt with the presidential transition which, as directed by the new Secretary of Energy, is a transition to a new culture based on consensus. We belleve, as we discussed in our proposal, this dramatic change in DOE is also motivated by the course of history and evolution of the world around us. So, we've participated in and observed the transition to a new administration and to a new culture. We wanted to set up opportunities to not just study some incidental consensus, but to study the consensus that is going to be the heart of DOE's change in culture. To get in touch with this cultural change, we've been heavily involved in the Five-Year Waste Plan, which will embody and direct more consensus activities.

To play the kind of role we need in the consensus activities and to make the contacts with the potential stakeholders and have their confidence, we not only need to observe and analyze them, but we also have to identify, meet, and interact with those stakeholders. We've set up 
relationships with people inside DOE and in DOE support agencies, such as other federal agencies, contractors, and state organizations. We want to understand the right purposes and the right participants for consensus activities. This networking activity will be the foundation for and key to the success of our test site effort area. We've worked on criteria for getting the right people to participate in consensus. We began to establish how we should 1) make measurements, 2) participate in significant consensus directing and geserating activities, 3) set up consensus gathering groups, 4) explore the types of meetings that these groups will participate in, and 5) survey the information systems the people in environmental management and protection are going to use to provide the facts they need for conducting successful consensus activities.

For the first grant year of our Consensus Grant, we were supposed to emphasize consensus process and consensus tools for rules and methods and to begin our literature search. This emphasis for the first grant year and the direction for the out-years is shown in Figure 2, taken from our proposal. This figure displays our five-year plan for accomplishing the objectives of our research in the six effort areas of our research concept shown in Figure 1. Figure 2 not only displays the shifting emphasis within the effort areas, but the relationship of two effort-supporting laboratories and research management support during the five-year period. Whereas the activities in the effort areas work together to accomplish the research objectives, the laboratories and research management support the effort areas.

We started our literature search ahead of schedule, but were also fortunate to have some preliminary definitions for consensus, types of consensus, and consensus related activities. We needed this information to support us as we began to deal with the data from all the consensus groups. We began the test sites far ahead of time. The test sites are the composition of, agenda for, and operation of the consensus groups. So, to get the most out of the test sites and the consensus groups, we emphasized data collection in the second grant year. We collected data earlier than we planned because of the opportunity of having test sites up and operating so fast. We re-oriented our plans so we're working in the test sites and gathering data before we have our frameworks and models fully developed. We couldn't let our proposal phasing get in the way and end up having the consensus groups possibly finishing their activities without our having made measurements. 


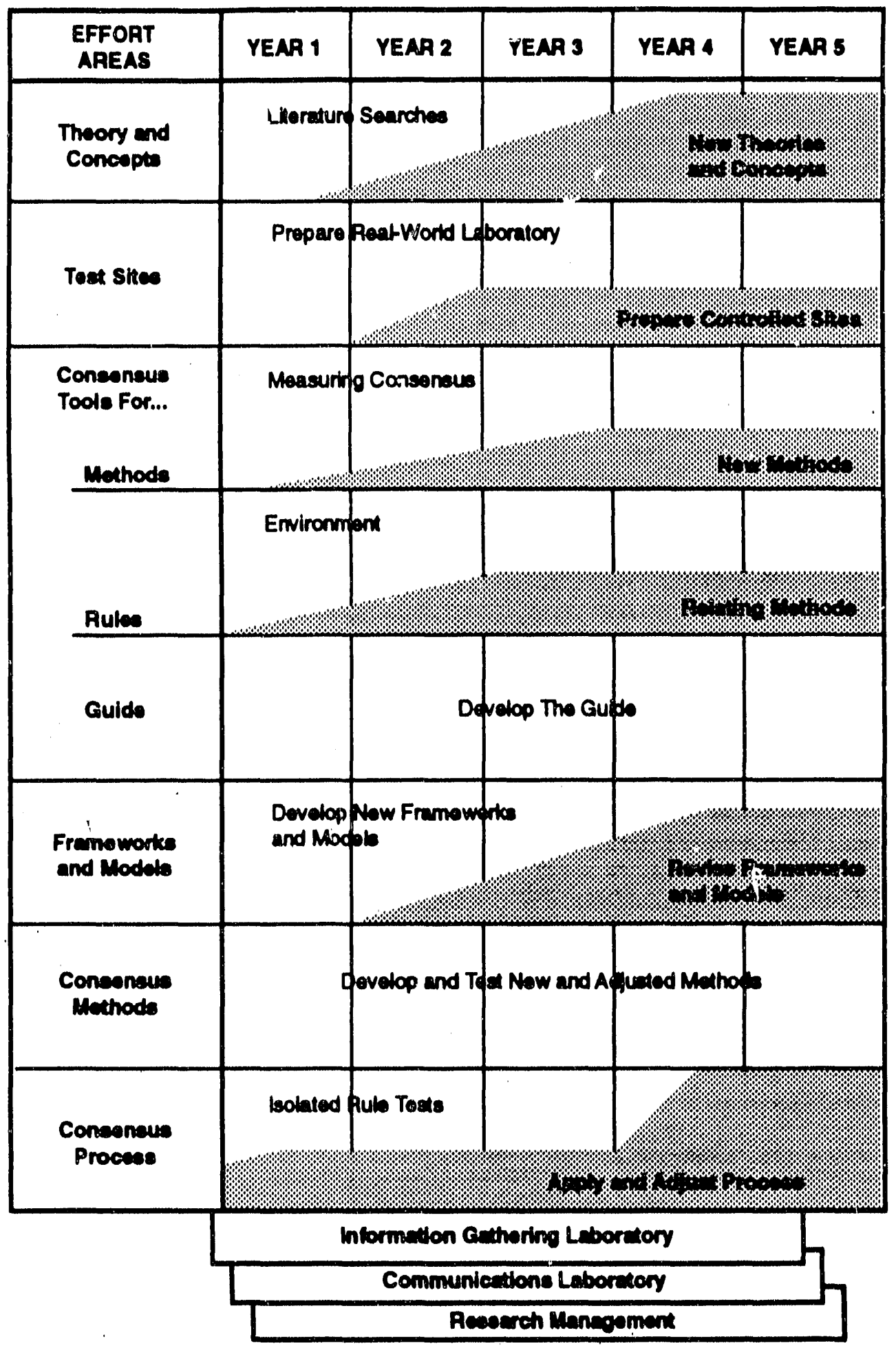

Figure 2. The research plan establishes annual objectives for each effort area. 
We plan to execute and report the research in terms of three "research domains"--one domain of fundamental research and two applied research domains. We've mapped the six effort areas (from Figure 2) into the three domains shown below:

I. Fundamental Research Theory and Concepts (exploratory)

Consensus Tools for... Methods (specific confirmatory) Rules (specific confirmatory) Guide (global confirmatory) Frameworks and Models (exploratory)

II. Consensus Processes Test Sites (specific confirmatory) Consensus Tools for... Rules (specific confirmatory) Guide (global confirmatory)

Consensus Methods (exploratory and specific confirmatory)

Consensus $\mathrm{P}$ rocess (specific confirmatory and global confirmatory)

III. Information Systems

Test Sites (specific confirmatory)

Consensus Tools for... Methods (specific confirmatory)

The three donains help us organize our research staff by area of expertise. The consensus tools effort area is broad and parts of it map into all three domains. Also, the test sites effort area is important to both those studying the process conducted at the test sites and those studying the information needed to support the process at the test sites.

The parentheses indicate the type of research we expected to dominate for the first grant year. For example, specific confirmatory research under consensus tools for methods means we expected to make measurements and do statistical analyses on whether a given tool affects the consensus of a group. The global confirmatory research type applies to the guide and somewhat to the end of the consensus process effort areas, and we won't get into that type of research until the end of the grant. Global confirmatory research is an integrative effort, bringing the results of the specific confirmatory research efforts together.

From Figure 2, we planned to emphasize the following affort areas, and therefore the related domains, during the first grant year.

I. Fundamental Research

Theory and Concepts (exploratory)

(literature search leading up to frameworks and models in the second grant year) 
Consensus Tools for...

Methods (specific confirmatory)

(measurements benefiting from previous studies of the environment)

Rules (specific confirmatory)

(environmental issues affecting test sites, consensus methods, and consensus process)

II. Consensus Processes

Consensus Tools for...

Rules (specific confirmatory)

(relate environmental issues to the consensus process)

Consensus Methods (exploratory)

(observe and interpret meeting dynamics of early consensus groups)

Consensus Process (specific confirmatory)

(test some consensus tools for rules in isolated

cases)

III. Information Systems

Consensus Tools for...

Methods (specific confirmatory)

(evaluate measurements in isolated cases)

Obviously our plans had to change because of the opportunities afforded by the presidential transition. We had to emphasize the test site effort area at the expense of the other effort areas. We've spent 158 of our funds on the effort areas in the Fundamental Research domain and 858 on the other two domains.

We didn't start the consensus tools for rules effort area as soon as we had planned. (See Figure 1.) However, we got a headstart in the theory and concepts effort area, on the literature searches. We're far ahead of our first grant year plans for test sites. We also got the opportunity to visit all DOE sites and do our information system analysis.

Domain I for the first grant year (reflecting the changed plans) is dedicated to 1) initiating the literature search by doing the pre-search and identifying disciplines, 2) defining consensus and the dependent variables of consensus, 3) developing taxonomies for different types (intra-, extra-, and inter-group consensus) and levels (coercion, compulsion, etc.) of consensus 4) slicing out the parts of the disciplines we' 11 apply to the frameworks and models, 5) evaluating environmental issues relating to the operation of consensus groups--use networking, 6) establishing or focusing the expertise to carry out all three domains, 7) deriving the factors needed to support consensus, and 8) presenting initial concepts for critical review (papers and presentations).

Domain II for the first grant year is dedicated to 1) identifying consensus group purpose and participants, 2) networking within DOE and other agencies and contractors so we can evaluate the roles of 
participants, 3) determining long-term, critical-path resources for all domains, 4) analyzing and participating in the presidential transition and how that relates to a change in culture, 5) participating in the structure and form of the Five-Year Waste Plan and how it relates to consensus, 6) setting up consensus groups for the long term so we can study them in longitudinal studies, 7) evaluating the effects of culture change (to a consensus orientation) on the organization structure as an example management tool, 8) developing methods for transferring information within the organization and in parallel organizations, especially during transition, and 9) presenting initial concepts for critical review (papers and presentations).

Domain III for the first grant year is dedicated to 1) determining the equipment and resources necessary to support the information gathering and communications laboratories, 2) initiating a survey of the status of information systems used in the fleld to characterize and track waste, 3 ) establishing contacts (networking) among field sites and headquarters to identify strengths, weaknesses, usability, and relacionships among information systems, and 4) presenting initial concepts for critical review (papers and presentations).

Fundamental Research

\section{Background}

We defined three different types of consensus: intra-group, inter-group, and extra-group. You might ask, "Why separate consensus into different types?" From our preliminary literature search, we've found consensus is thought of as a state among individuals. Consequently, measurement techniques have been derived to measure consensus among individuals (intra-group consensus). We believe, however, there are consensus situations where these methods are applicable but not feasible due to their complexity. In some environments, especially DOE, consensus is necessary among groups of individuals, or agencies (inter-group consensus). When trying to measure consensus between groups of individuals, the methods commonly used are too complex. Consensus is also necessary between DOE and its external environment, the publi,c (extra-group consensus). We recognized there are different types of consensus which arise from different situations and must be measured in different ways.

We defined intra-group consensus as a state achieved between three or more people after sufficient discussion has occurred for every individual to voice their opinion. Each individual then accepts the outcome which is not the same as any one individual wanted going in. Consider Galbraith's explanation: "The problem solving approach [to group decision making] is intended to achleve a consensus which obviates the need for a powerful leader. Consensus is not unanimity but a state of affairs in which the individual who disagrees with the preferred solution feels as follows: 'I understand what most of you would like to do. I personally world not do that, but I feel that you understand what my alternative would se. I have had sufficient opportunity to sway you 
to my point of view but clearly have not been able to do so. Therefore, I will go along with what most of you wish to do."

This type of consensus deals with human interaction on the micro level. It deals with small group interaction and decision making.

If we try to measure intra-group consensus as a state variable describing the degree to which a decision is carried out, we risk measuring the wrong indicator of consensus. In the intra-group situation, a decision is made irrespective of the ability of the group to carry it out. Therefore, measuring the extent to which a decision is carried out may only be indicative of the power the group wields within the organization, not the actual consensus achieved in the intra-group situation. To measure intra-group consensus, you must deal with the human aspects of decision making and interaction.

We believe to achieve consensus, a group must transform from a nominal group (a group in name only) to a real group. This transformation requires the raising and resolution of underlying conflict among group members. That is why we believe the Nominal Group Technique, for example, is better for information sharing than it is for deriving consensus.

We defined inter-group consensus as the degree to which two or more groups (who have achieved intra-group consensus) within a larger group or organization, agree on actions to take place or issues of importance to the organization. This type of consensus, unlike intra-group consensus, deals with human interaction on a macro level between two or more independent groups or societies. This is the type of consensus the different government oversight agencies need to coordinate their actions.

We believe inter-group consensus can be measured by the actions taken as a result of the consensus achieved between groups. When dealing with groups, we can model each group's preferences, values, and opinions as a single point and then proceed with the same methods used for intra-group consensus. This would not be very accurate however. When two or more groups of individuals are asked to come to consensus, we assume their consensus is to relate to some course of action. This allows us to measure the strength of inter-group consensus by measuring the degree to which consensus decisions are carried out (regardless of quality).

An important point must be made regarding the measurement of consensus. The choice of using either interaction methods such as those proposed for intra-group consensus or activity methods such as those proposed for inter-group consensus really depends on the power of the group to carry out any decisions made. If a group has the power to implement their consensus decision, intra-group consensus can be measured with the methods proposed for inter-group. Likewise, if the groups coming to inter-group consensus don't have any power but are acting in an advisory role, then inter-group consensus must be measured with the methods proposed for intra-group consensus. 
It's not clear conflict resolution is needed to get inter-group consensus when the groups are advisory and power isn't a question. We have set up a consensus group called the Environmental Management Review Group. This group plays a consultative role and as such can't have its consensus measured by the actions carried out as a result of their recommendations. To do so might measure the power of the group members or the quality of their recomendations, but it definitely would not measure the degree of consensus achieved by the group members. DOE is made up of many groups which must act in crincert with each other. Therefore, they need inter-group consensis on the actions taken within DOE. Inter-group consensus, in this case, is best measured by the extent to which these actions are carried out.

Extra-group consensus is a state achieved between one or more groups in an organization and one or more groups in the external environment outside the organization. The difference between extra-group and intergroup consensus is that for extra-group consensus the group in the external environment doesn't have the power to directly affect the actions of the domain or organization being analyzed. The organization, however, needs acceptance of the external group to be most effective in carrying out their actions. For example, if we look at EM, inter-group consensus is achieved between the oversight agencies and extra-group consensus is achieved between GOA's and DOE. If, however, we look at DOE, the consensus achieved between GOA's and DOE is a higher level of inter-group consensus than the consensus achieved between the GOA's. Extra-group consensus in this case is the consensus achieved between the GOA's or EM or DOE and the various groups making up the public of the United States.

DOE has often acted without considering the wants of the public or states affected by their decisions. They have tended to bring in the affected outside parties after the fact--after decisions are made--to get their reactions, not to involve them in the decision making process from the beginning.

In the case of the Environmental Management Review Group (EMRG), by the definition we just proposed, the unit of analysis must be DOE. EMRG was set up to represent a range of stakeholders--together representing the public. Therefore, the outside group is the public and the organizational unit of analysis must be DOE. We're looking at extragroup consensus between EM (a group within DOE) and the public of the United States. Consensus between EM and DOE on any actions taken as a result of EMRG recommendations will be inter-group consensus. In actuality, EMRG will come to intra-group consensus among themselves, but EM is using them to model inter-group consensus among public interest groups. If EMRG's intra-group consensus doesn't accurately model intergroup consensus among public groups, the extra-group consensus between EM and the public will be weak or non-existent. By setting up EMRG, DOE is attempting to get information on how to involve the public earlier in the decision making process. This attempt moves DOE further toward the goal of including stakeholders at the beginning of the decision process. It is not enough, however. 
This type of measurement can't be measured by any of the methods discussed so far. Since an external group has no direct say in any actions taken within the domain being considered, extra-group consensus can't be measured by any actions taken within that domain. This doesn't mean ar external group can't influence the actions taken within an organization. They can influence actions through pressure, lawsuits, lobbying, etc. They don' $t$, however, have the ability to directly tell the organization what they should do. We think, to measure this type of consensus, you need to measure external groups' reactions to the actions taken as a result of inter-and intra-group consensus. In the case of DOE, extra-group consensus with the public might be measured indirectly by the response of Congress to their actions since Congress is supposed to represent the public. It might also be measured by reactions printed in newspapers or demonstiations by public groups.

Another important point we must make is that consensus is not a discrete variable. It is a continuum encompassing different levels of interaction. If we just consider measuring consensus by the actions taken as a result of the consensus to say "yes we have consensus", or "no, we don't have consensus", we're looking at consensus too narrowly. If we want to look at consensus as a group acting as one, we must differentiate different levels of consensus. It is too simple to consider a situation where people are coerced into certain actions on the same level as people who freely agree to act in a certain way. We can say that consensus is achieved in each of these situations, but we must $\sigma^{2}$ further and define the level of, or strength of, the consensus.

The differenze between types and levels is this: a level is a measure of the cognitive aspect of consensus and a type is a classification of the consensus situation. A level is a point on a continuous scale, a type is a discrete situation. Each type of consensus can have any level of consensus.

The strength of consensus is a combination of cognitive and behavioral factors. To quantify the cognitive factor, consider five levels of consensus along a continuum as follows:

coercion compulsion acceptance agreement unanimity

CONSENSUS

Figure 3. Consensus can be pictured as a continuum.

We define these terms in the following way:

* coercion - one person defines the behavior (idea, plan, decision) and has power to, and uses power to force other group members to accept the behavior. 
* compulsion - one or more people (the total being a minority in the group) are the main drivers of the behavior (idea, plan, decision) while the others "go along".

* acceptance - one or more members find the behavior (idea, plan, decision) undesirable but will go along with it.

* agreement - the behavior (idea, plan, decision) has support of the entire group although it is not the ideal behavior for some or most of the members.

* unanimity - all members unanimously and enthusiastically agree on the behavior (idea, plan, decision) and support it.

Consensus isn't a discrete variable. It's a continuum encompassing different levels of interaction. We must realize that in each of the levels defined above, actions are taken which lead us to conclude consensus was achieved. The strength of the consensus, however, is vastly different in edch of these situations.

Strength of consensus is some combination of the attitudinal and the behavioral sides of consensus. A group can leave a discussion thinking they have achieved unanimity (regardless of whether the decision is of good or poor quality); but, if nothing is done to implement the actions declded upon, the consensus was very strong attitudinally but very weak behaviorally. If we combine these two parts of consensus multiplicatively, the consensus achieved is very weak. Likewise, if a group leaves a discussion and the members are angry and very factioned but they carcy out the behaviors agreed to, the consensus is weak from an attitudinal standpoint but strong from a behavioral standpoint. The overall consensus achieved is then weak. The only way to assure a strong and lasting consensus is to achieve both a strong attitudinal and behavioral consensus.

One area where consensus is important in an organization is to agree on the information requirements that the organization needs. The reason you need to agree on the information requirements is: if you have limited rescurces, you can improve, computerize, or move forward only on some of the information requirements but not on all of them. So what you want to do is agree on the relative importance of the various information requirements. There are several ways to do this. The typical way is to bring in the information specialist unit in your organization. Have them conduct interviews, which is one way of trying to derive consensus, talk to all the stakeholders, meld everything together into an approach, and then return to the stakeholders for their concurrence on something like an information requirements document. The problem is, it takes a very long time to gather the information, produce the document, and get concurrence. In the meantime, everything has changed. Information requirements themselves have changed. So it's very difficult to keep up. Also, developing information requirements is considered a support activity and not a line activity, so it's hard to get people's attention. Therefore you don't get people's full attention on the information requirements document. 
Another approach to getting consensus on information requirements is to bring stakeholders together in a workshop setting. The stakeholders include people with information requirements and the information specialists. In a workshop setting, the stakeholders can 1) concentrate on this support activity, 2) interact, and 3) move forward quickly. You would use consensus techniques or tools in the workshop to try to come to consensus. You'd also use information systems techniques in the workshop. The workshop then includes a structured approach to getting consensus and a structured approach to identifying information.

We began a comprehensive literature review for our consensus library, The literature review includes three major elements: 1) a library search, 2) analysis of the literature, and 3) preparation of a written summary of the findings. We planned the literature searchi in three stages. Stage one, the pre-search, is broad and shallow. Stage two, the discipline-oriented search, is less broad but much deeper. Stage three, the paradigm-oriented literature search, is very focused. The major outcome of this effort is the development of new theories, concepts, and models designed to support our research on consensus.

The purposes of stage one (the pre-search) are to define relevant disciplines, to identify key journals, and to list key words. We want to get a feel for the size of the literature involved, the number of disciplines involved, and the number of different types of journals. We need to determine the relevant time period over which people might have written relevant articles and books. And we need to figure out the key words to help us do a better search of the literature.

After we daveloped a set of key words from the manual pre-search, we worked with a reference librarian in Virginia Tech's Newman Library on the next steps for a comprehensive, multi-disciplinary literature review. We completed an electronic search using the DIALOG Information Retrieval Service, accessing 280 data bases in a brozd scope of disciplines. We used the key words identified during the pre-search to conduct the electronic search.

We identified 112 articles from the DIALOG search. Several researchers have begun reviewing these articles along with the articles identified during the manual phase of the pre-search. We designed a standard Literature Review Form to use when summarizing articles. These summaries will be entered into the MSLSearch computerized data base and reference system using a data base (Composinet) designed by MSL for another project. This reference system will be utilized by project staff, students, and MSL researchers when writing papers or presentations.

Accomplishments

For fundamental research efforts, we started four studies during the first grant year. We added seven more studies before the end of the first grant year. The seven new studies are 1) consensus process studies, 2) consensus paradigm development, 3) consensus academic plan, 4) consensus case study research, 5) consensus gathering systems, 6) consensus group dynamics, and 7) information avallability. Therefore we 
worked on eleven studies in fundamental research during the first grant year. Since the grant began after the beginning of the 1988 academic year, most graduate students had already committed to other funding. To get the kind of graduate students we wanted with the right background and interests, we delayed getting a number of graduate students. At the beginning of the 1989 academic year (August), we added three new graduate students. Obviously, this delay in obtaining graduate students delayed preparation of presentations and papers.

In Consensus Library, we began identifying and searching all the relevant disciplines for articles related to consensus. After examining all the disciplines, we reduced the numter of disciplines to eight. We identified some key words and varlables but didn't begin to summarize the literature. Plans were made to set up the library using a data base called Composinet. This on-line reference system is an interactive data base, so everyone at MSL can have access to the 1iterature.

In Consensus Process. Studies, we identified and defined different types of consensus: i,atra-group, inter-group, and extra-group. In this exploratory stage of our research we took the concept of consensus and began analyzing its factors. We began diverging and exploring what relationships we want to look at. We started identifying methods for measuring consensus such as using interaction methods for intra-group consensus or activity methods for inter-group consensus. We also looked at what variables affect choice of measurement tool.

In Consensus Paradigm Development, we began identifying the elements of consensus by examining information collected through the disciplineoriented literature search. As we attempt to build a theoretical model, we'll include such elements as problem, participation, people, process, purpose, and product. In order to fully understand consensus and to enable us to structure the remainder of the literature search, we'll continue to collect variables and try to determine their relationships.

In using NGT for Information Requirements, we began studying the feasibility of using data flow diagrams or something like data flow diagrams in a workshop setting using elements of Nominal Group Technique (NGT). We made plans to test a modified NGT as a means of getting agreement on information requirements.

In Organizational Statics and Dynamics, we studied theory and concepts addressing the issue of organization change and how the organization adapts to it. We focused on relating an organization's success or failure to its adaptability to change. We studied Kotter's Model (1978) of organizational dynamics and how it matches our concept of consensus as a state varlable and confirms our sense of GOA's' need for flexible consensus maintenance. We began refining the definition and different types of consensus, as well as, researching the varlous ways we can measure consensus.

In Consensus Academic Plan, we began developing our five-year plan to coordinate our research efforts in the three research domains. This plan will be revised and updated each year as we feedback our research findings. Thus our plan is a living plan. 
In Consensus Case Study Research, we determined speciflc historical areas involving consensus processes. We researched many areas in which consensus or lack of consensus may have been a determining factor of the outcome. The areas we chose were 1) Presidential advisory groups ("kitchen cabinets"), 2) labor/management negotiations, 3) emergency relief, and 4) foreign policy/peace negotiations. We did case studies on several specific situations in each of these four areas. While the discipline-oriented literature search produces the academic framework for studying consensus, this research concentrates on application of consensus. We'll use our results to support our consensus paradigm.

In Consensus Gathering Systems, we started developing a system that accepts the ideas of group members individually, understands areas of agreement among the ideas, works on compromise and support, and identifies the degree and breadth of consensus. In the real world, when we have several people who disagree but discuss the problem, we get a moderating effect that yields a consensus solution. A consensusgathering system is an expert system that captures the benefit of that moderating effect. This system has the ability to understand what the users say, extract the user's ideas from what they say, identify similarities and differences between the ideas, and set-up a hypothesis as a consensus among the group.

In Consensus Group Dynamics, we used information from the disciplineoriented literature search to begin documenting variables related to group dynamics. We narrowed our research interest to group commitment. This study wis in its early stages at the end of grant year one.

In Information Availability, we began studying the relationship between information availability and consensus. We want to determine whether or not consensus is desired and/or can be reached under certain information circumstances.

Presentations and Papers are included in Volume II, Papers, Presentations, and Conferences.

Consensus Process Studies

\section{Background}

We researched the factors required to gain consensus and developed a framework. The framework starts by adapting only the words of the equation: Purpose + Audience - Design. This framework is pictured in Figure 4. Replace the word audience with participants. Then for a good meeting, or to achieve consensus, we need to begin with knowing who the participants are and what the purpose of the meeting is. The design would yield two more $P^{\prime} s:$ 1) process and 2) presentation and/or portrayal. So if we know the purpose and the participants in the meeting, we should be able to determine the best process--like NGT, for example. We should also be able to determine how we should present the information among participants. The result of the process and the presentation is the fifth $P$ : product. That is to say, for any meeting, 
or any time we want to achieve consensus, we should have a tangible result or product, such as a decision we can write down or a list of action items in rank order.

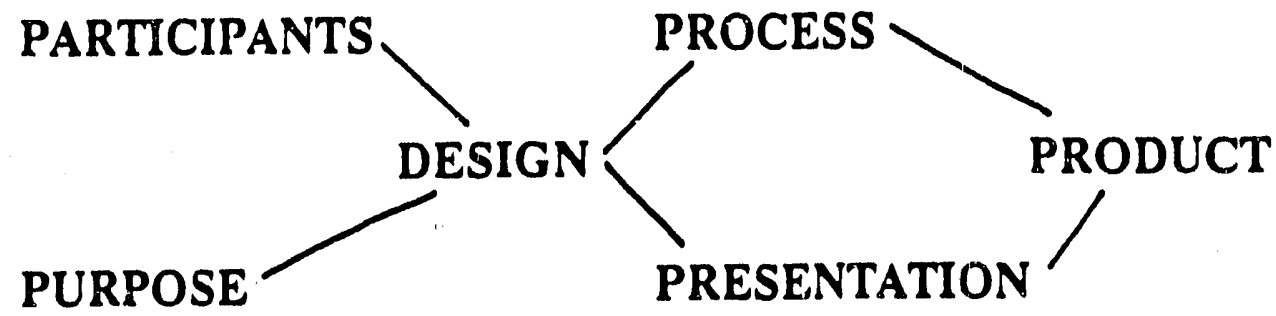

Figure 4. There are certain factors required for supporting consensus.

In summary, the five $\mathrm{P}^{\prime} \mathrm{s}$ are purpose, participants, process, presentation and/or portrayal, and product. The meeting facilitator must consider all five $P^{\prime} s$ in developing a meeting, conducting a meeting, and evaluating the success of that meeting.

EM uses management tools to support decision making. Environmental change requires new management tools. To deal with Admiral Watkins' change in culture toward a consensus orientation, EM (or any organization affected this way) must change Its management tools. One such management tool is the organization structure. To effectively manage in the new culture, EM should consider how it should reorganize to implement the consensus-oriented culture. (The Secretary will cause the reorganization to happen.) In a like manner, EM should consider changing other management tools, like their information systems and their plans.

We believe there are rules and steps to follow when reorganization takes place: Rule one: Your organization structure is for carrying out your strategy for dealing with your environment. That is, if your environment changes (internal or external, e.g., you found new techniques for doing your fob or someone is changing the culture), then you need a new strategy for dealing with that new environment. Your organization structure must then be adjusted to carry out the new strategy. Rule two: Fit your people to an organization structure; don't design a structure to fit or suit your people. These rules assume ideal conditions (e.g. you have the ability to gather the people you need to fit your organization structure). So, we can evaluate a set of steps that start with the ideal and fold in the practical.

We've tried these five steps before in organizations with some success. Step 1: Figure out an ideal organization structure using rule one above. Step 2: Develop a set of organization structures evolving from what you now have to the ideal. Step 3: Pick the structure from step 2 you think is most feasible for practical considerations. Step 4: Fill in the details (titles, levels, etc.) of the organization structure recognizing the people you have available. Step 5: Write position descriptions for the organization structure. 
The test sites for studying consensus and measuring variables of consensus should be groups of people trying to achieve consensus (intragroup consensus). Better test sites are groups that come to consensus over the same issues (inter-group consensus) and groups chosen to best represent the public (extra-group consensus). An example of such a group for gaining consensus and relating to other groups and representing the public is the Environmental Management Review Group (EMRG).

We call the EMRG our Consensus Group 1. It's a review group designed to provide independent and objective review of the U.S. Department of Energy's (DOE's) Office of Environmentai Restoration and Waste Management's (EM's) activities. The EMRG provides independent review, analysis, and support for policy, program, and strategy issues in EM's management of radioactive and hazardous waste and environmental protection and restoration.

The EMRG will provide the following (Purpose from Figure 4):

* objective reviews, evaluations, and assessments of current plans, projects, and activities related to EM policies and actions mandated by Congress, the Executive Branch, and the DOE; and

* research, analysis, and communication necessary to substantiate the reviews, evaluations and assessments and provide useful information, conclusions, and recommendations to EM.

Members of the EMRG (Participants from Figure 4) are selected on the basis of specialized experience, credentials, or background relevant to th. EM-related activities. The EMRG members serve on a rotating basis for a term up to three years; the size of the EMRG is 1 imited to ten members. Membership appointments are staggered so approximately two or three members change annually.

EMRG members must be familiar with laws and government policies and current issues in the management, remediation, and transportation of radioactive and hazardous waste. At the same time, EMRG members must provide objective review. Therefore, all EMRG members complete and return a statement regarding their past and present contract work and/or employment which may tend to affect their objectivity in reviewing and commenting on sponsored activities. In addition, each EMRG member is under a continuing obligation to report to the EMRG chairman subsequent activities which may impair the EMRG member's objectivity regarding sponsored activities.

The following paragraphs relate to the factors of Presentation, Process, and Product of Figure 4. The chairman proposes meeting schedules and agendas, presides over meetings, calls for special meetings, and sets attendance. The EMRG documents each meeting with minutes. The minutes are a summary of the highlights of the conducted business. Draft minutes are issued for: review and approval by the EMRG. The EMRG approves previous minures at the start of each meeting.

The EMRG uses two methods to meet its objectives. First, the EMRG generally reviews current laws, policies, and issues affecting EM. 
Second, the EMRG revlews specific issues at the request of MSL. The EMRG's contact with individual sites or organizations or with individual research project personnel is coordinated through MSL. This is not meant to inhibit interaction of individual EMRG members with individuals of the various affected groups.

The EMRG meets approximately once each quarter. The EMRG recommends meeting schedules for MSL approval. In addition, the EMRG may meet in special session with a full or abbreviated membership to consider specific topics.

Another test site, or Consensus Group 2, is the Institutional Group. Now called the state and Tribal Government Working Group (STGWG), we helped identify its players. This group will evaluate the drafts of the Five-Year Waste Plan for DOE, as well as the final plan. The Five-Year Waste Plan is a plan being written to define what DOE is going to do with their waste during the next five years. STGWG consists of representatives from selected states, tribal governments, and public interest groups. The membership was chosen by Admiral Watkins. For meetings in June and July, we invited gubernatorial representatives from nine states (Colorado, Idaho, Kentucky, Nevada, New Mexico, Ohio, South Carolina, Tennessee, and Washington) with major DOE facilities in them. We also invited two Indian Nations (Yakima and Shoshone/Bannock) with major DOE facilities on their reservations and representativas from the National Governors Association, National Council of State Legislatures, and the National Association of Attorneys' General. We chose these participants because we thought they would be the most interested in DOE environmental management and cleanup activities.

MSL's Director is the facilitator of the STGWG meetings. For each of these meetings we determine what consensus techniques to use depending on the issues to be addressed. We want consensus on the generic issues to the states. On other issues we only need to share information.

The relationship between the STGWG and the DOE has been successful and highly beneficial to both. The DOE has made a significant commitment to the STGWG and has tried to incorporate changes they suggest in the FiveYear Plan. Throughout the meetings, Secretary Watkins remained in close contact with DOE personnel so he could provide real-time response to STGWG comments.

\section{Accomplishments}

For consensus process studies, we started three long-term/ongoing studies and eight short-term studies. We added one new long-term study and three new short-term studies by the end of the first grant year. The new long-term study is Consensus Group 3. The three new short-term studies are 1) nuclear waste transportation, 2) regulatory consensus research, and 3) five-year research and development. Thus we worked on fifteen studies during the first grant year. Our subcontract with UME helps us to identify the best participants for our review groups and to become involved in critical consensus activities like the Five-Year Waste Plan--in short, UME helps us network. 
In Management Analysis and Networking, we spent a tremendous amount of time bringing together the right people at MSL and making the right contacts in DOE and DOE's support agencies. We needed access to the Five-Year Waste Plan and access to the people who will evaluate the plan.

We also began studying the present culture of DOE and steps they need to take to initiate their new consensus oriented culture. We'll work on the design and research of a consensus building tool with the help of DOE experts, consensus experts, and organizational experts. When AT\&T was proactive in changing their culture, they developed the "shared values pyramid" as such a tool.

In Consensus Meetings, we began to set up and study various consensus groups. Fortunately, DOE asked us to establish some groups that will put us in a position where we can make all the measurements we want. Our interest is in measuring the groups as they're interacting, not in the logistics of the group. However, you can't separate one from the other very well. So, we're trying to initiate the groups, 1.e., bring together the right participants.

Another issue is to have the right process to get consensus and to get the right product out of the consensus. We're dealing with variables such as process and product for consensus-oriented issues like the FiveYear Waste Plan. Once the group is up and established, the meeting times, room reservations, etc., are necessary to studying consensus, but that's not where we want to spend our research funding. So, once these groups are established and the first meeting is implemented, we'll turn the logistical requirements for those groups over to another organization. We'11 influence their charter or establish an understanding that we' 11 be attending the meetings and making measurements.

So the idea on the consensus meetings is: What sorts of techniques or things do we want to do in those meetings? Do we want consensus? What do we want to measure? Our objective is doing the design, understanding that activity, and measuring the results of that activity. Essentially, consensus meetings deals with the factors in Figure 4 after the design; namely, presentation, process and product.

In Consensus Group 2, we formed an ad hoc States review group, now called the State and Tribal Government Working Group (STGWG) to comment on DOE environmental management and cleanup activities. This study has significant potential to help resolve many of the controversial and contentious issues surrounding cleanup of our nation's nuclear defense facilities. We invited several people to attend the Waste Management ' 89 Conference in Tucson to get their feedback on the development of this group. Essentially, the Consensus Group 2 (and Consensus Group 1) deals with the factors in $F^{\prime}$ gure 4 before the design; namely participants and purpose.

We initiated the first meeting in June and another in July. We designed a process where the group was free to go into executive session, which the group chose to do. The executive sessions were intended to 
represent inter-group consensus among the constituents of the group. After interviewing members, we believe the group nay have achieved intra-group consensus as well. Whether or not the executive sessions turned into extra-group consensus between DOE and the STGWG wasn't as important as the STGWG members feeling their suggestions were seriously considered. The STGWG chose to speak to DOE with one voice after these executive sessions.

In Consensus Group 3 (Interagency), we began identifying groups that represent an entirely different type of stakeholder (agencies outside of DOE or public interest groups) from the other consensus groups we've studied. For instance, we are interested in studying the consensus process as DOE interacts with other federal agencies.

In Transition Management, we studied EM's needs for new forms of information portrayal for presidential transition activities. We used the data collected to design a modular overview briefing and video addressing issues specifically for transition and for general purposes too. Part of the new Secretary of Energy and the change in culture towards consensus is the transition of that new Secretary and his people into the organization. We've worked with that transition in general, and the cultural transition in specific, in terms of how to manage it.

In Continuous Information Transfer (Video), we analyzed EM's budget and updated portions of the video to reflect EM's current budget program. Our results can be used to develop and evaluate other media. Continuous information transfer is designed so $1 t^{\prime} s$ not necessary to have a person present for the information portrayal.

In Discrete Information Transfer (Modules), we used the data we collected to give MSL tools to research, gather, and structure raw, informal data into formal, meaningful data. These data can be shared within EM and across EM into other organizations. MSL's analys is will identify the communication purposes of the EM transition briefing, the intended transition audiences, and any constraints on a transition process. The results of this analysis can be used as a standard to develop and evaluate transition media.

In Consensus Group 1 (EMRG), we researched with academicians and industry experts the development of a Environmental Management Review Group for EM's EM-40 efforts. We drafted a charter and screened, selected, and invited potential candidates for membership. We facilitated the first meeting of this group in Blacksburg during February to review the Program Optimization System (POS), the issue of privatization, and EM environmental management fellowships. We took one day for presentations (one way information direction) with clarification questions. The second day was spent in deliberation. The consensus that came out of that meeting was not the first day and not the second day. The consensus came about when the facilitators (MSL) went out and gathered, organized, and structured the deliberations of the second day into a document. That document was circulated twice to all the members of the EMRG for their input, and a follow-on meeting was held in Washington to agree on the final form of that document. That was intragroup consensus. However, DOE disagreed with the results of that 
document, so we didn't achieve extra-group or inter-group consensus out of that meeting.

We facilitated meetings in May and September. The September meeting ran smoother and was more productive due to what we'd learned about improving and catering different processes to different groups. Our experience directed us in asking DOE to present problems and let the EMRG make positive recommendations on solutions. This process worked much better and both the DOE and EMRG found the meetings very successful.

In Coordinating Information Transfer in Parallel Organizations, we studied the needs of OMA and used our data to produce transition materials for General Kavanaugh. We'll use our analysis in future information portrayal research.

In Nuclear Waste Transportation, we began looking for opportunities to study and participate in consensus processes occurring between DOE and local governments. The issue of transportation of waste creates some highly opinionated and emotional situations. As DOE moves toward a more open policy, we plan to observe meetings set up with localities to deal with the issue of transportation of nuclear waste.

We coordinated and hosted a National Conference in September which focused on the themes of nuclear materials transportation, emergency management, and federal/local government cooperation. This provided us a unique opportunity to observe exchange of ideas and the interaction of a diverse group. The participants included senior officials from local governments from all over the country.

In Regulatory Consensus Research (Land Disposal Restrictions), we began researching land disposal restrictions. We'll research the guidelines by EH and the regulations determined by the EPA. Our research will provide a consensus tool for Environmental Restoration and others to use and apply to disposal of waste.

In Five-Year Waste Plan, we participated in the shape and form of the plan. It's form is designed to promote understanding so people of different backgrounds and different desires and agendas can do what they need to do with the plan. MSL's influence produced more of a plan versus a status report. We don't believe consensus would be worth studying if the plan was a status report.

Instead the Five-Year Waste Plan addresses the issues people need to come to consensus over. Then you need consensus and have a purpose for consensus and you can study consensus. We continued to influence the drafts and the final plan during the first grant year. We were involved in the research of who would review the plan and who the stakeholders were. We also participated in site implementation plans.

In Five-Year Research and Development, we appruached the Five-Year Waste Plan from an additional perspective. We began a thorough investigation of pertinent data related to the planning process. We participated in 
the unfolding of the plan by researching management planning, structures, and reporting.

Presentations and Papers are included in Volume II, Papers, Presentations, and Conferences.

In Networking for Consensus (UME), we spent : lot of time setting up bridges and networks, making contacts, having discussions, interviewing people, providing information for those people, and convincing those people to participate. We conducted research to make sure these people got the right information at the right time to allow them to contribute significantly. This whole activity was designed to feedback information to us on the consensus of the networking.

Information Systems Studies

\section{Lackground}

We visited eight Operation offices and two National Labs (Lawrence Livermore, Argonne). Our purpose for these visits was to identify and document the information systems used in the fileld to characterize and track waste. For each waste tracking system, we identified the system scope, what data were collected, what reports were issued and to whom, and what other information systems each system provides information to. We also looked at who was accountable for waste tracking systems data, who provided $Q C / Q A$, and who uses the waste tracking systems.

We found the following ten generalizations to be true:

1) Almost all locations have waste tracking systems that satisfy their own needs and characterize waste according to DOE Order 5820.2A requirements. Those locations that currently do not provide the detail needed to comply with 5820.2A are planning information system enhancements or replacement. In addition, some information systems meeting $5820.2 \mathrm{~A}$ requirements are being upgraded to allow easier data entry, retrieval, and communication with other locations.

2) Waste characterization detall depends on when the waste was characterized. In general, waste currently being characterized meets $5820.2 \mathrm{~A}$ requirements. Waste older than approximately two years is not characterized in this detail. The older the waste, the less characterization is avaliable for the waste. Older waste is not being reassayed for recharacterization unless the waste must be relocated for storage or disposal.

3) Waste tracking typlcally begins when waste is placed in a container. When workers place waste into a container and inspect the container, they complete a manifest describing the container contents. This manifest is then entered onto 
a waste tracking system. Most waste is not tracked until this point.

4) Waste tracking systems vary according to their scope. Some Operations offices have systems that track all wastes for all generators reporting to the Operations office, while some have different systems for each waste type, each generator, each bullding/location, each contractor, etc. Having many information systems makes it difficult to trace the history of individual waste containers, to report aggregate quantities, and to answer informational queries. Some Operation Offices are consolidating waste-tracking systems into uniform site-wide systems.

5) Most Operations Offices want some Information guldance from EM HQ. Operations Offices want to know what the data they provide to EM HQ are used for. Many want EM HQ to define what information EM HQ needs so that planned fleld information system improvements can be designed to incorporate these needs.

6) Container tracking of waste throughout its iffe is difficult or impossible for some wastes. Waste tracking by container is difficult when waste is stored or discharged outside of a contafner. Container tracking is also lost through many treatment processes, such as incineration or vitrification.

7) There are no common definitions of waste and how it should be characterized. Lack of uniform definitions preclude the ability to generate complex-wide information like total mass, total radioactivity, etc. Standardized codes for waste characterization (transportation or ANSI codes) aren't descriptive enough. Current characterization varies from site to site according to regional requirements, local process requirements, and local policy. Volume reporting is not consistent and often depends on what volume is important to the people dealing with the waste at that time. For example, a waste generator is concerned with the volume of waste only, a waste disposer is concerned with displacement volume for burial. Most Operations offices see the need to standardize waste definitions and waste characterization information.

8) Informational roles/responsibilities vary among Operation Offices and among Lead Offices. Responsibilities and procedures for waste information reporting, QA/QC, audits, oversight, and informational inquiries vary among sites and by waste type. This lack of uniformity makes it difficult to identify individuals accountable for these functions at different locations.

9) EM Internal waste Information requirements differ from external requirements. Many sites have specialized information requirements imposed by the county, state, 
region, etc., they're located in. External regulatory agencies such as EPA, NRC, etc. also impose specialized waste information requirements. Many of these requirements are more stringent and/or are based on different assumptions than DOE's requirements. Therefore, tata in reports issued to external agencies may not be consistent with data reported to EM HQ. Inconsistent data gives the impression that EM does not do a good fob in managing its wastes.

10) Waste Informetion systems for OCRWM, ES\&H, EM, etc. are separate, even though they have overlapping data requirements. Each DOE HQ Office develops its own systems and policies even though responsibilities and waste data may overlap with other DOE HQ Offices. With many systems having common data, it's difficult to make sure data is consistent.

\section{Accomplishments}

For information systems studies, we started one long-term study and four short-term studies. We added one new short-term study before the end of the first grant year. The new short-term study is environmental

restoration and waste management data base'. We worked on seven studies in information systems during the first grant year. When dealing with getting the right information to decision makers involved in consensus activities, four considerations support information systems: 1) computer networks, 2) data bases, 3) performance evaluations, and 4) waste flow modeling. We explore these considerations in our short-term studies.

In Long-Term Overview Information Study, we surveyed the information systems of eight DOE sites and two DOE laboratories the managers use to gather information to make waste and environmental oriented decisions. We found more than 100 information systems and almost none of them related to any of the others. We began to analyze the implications of those systems today and in the future for environmental management in DOE. Studies included cradle-to-grave data flow analysis for all waste types and demographic data for all waste tracking/characterization systems. Our interest is in discovering the degree of consensus and consistency among and between waste information systems.

In WIN Interface studies, we planned to identify problems with WIN by analyzing the results of a review by the HAZWRAP/SCO group in Oak Ridge. MSL anticipates that some of the problems will be assigned to this study area as a task or set of tasks because of their role as consensus related issues.

In IDB/SWIMS Interface Studles, specific tasks weren't identified for this study. One task may be to develop a list of changes in IDB and SWIMS to bring them into compliance with regulations and orders to provide the right data to support consensus.

In A-106 Interface Studies, we looked at off-site reporting to EPA and $O M B$ on, specifically, waste milestones and budgets. We studied ways to provide reporting to external agencies so reports produced at various organizational levels are consistent. 
In RADTRAN Interface Studies, we studied the States' and Tribes' requirements against RADTRAN capabilities to determine what should be done to RADTRAN to make it acceptable. The assessment determined if the right information was presented for the states, Tribes, and DOE to reach consensus on the risk associated with shipping radioactive waste.

In Environmental Restoration and Waste Management Data Base (EMDB), we began to research the DOE-wide requirements for the EM data base. We plan to use the results to study the design of an EM data base for providing consistent waste data to stakeholders throughout the DOE waste complex.

Presentations and Papers are included in Volume II, Papers, Presentations, and Conferences. 


\section{SECOND YEAR PERFORMANCE REVIEW}

For the second year of our Consensus Grant we focused on consensus process studies and especially on the test sites, consensus methods, and consensus process effort areas. We continued to take advantage of the fortuitous events of the first grant year which supported our development of test sites and initiation of consensus groups.

There were no guarantees the Secretary of Energy would continue his emphasis on consensus groups for the lifetime of the grant. So, as we participated in and observed the workings of these consensus groups, we wanted to accumulate as much data as we could for our future research efforts.

We wanted to make sure the plethora of data, made possible by establishing consensus groups and test sites early in our grant, were the right data. This was hard to do without the benefit of a complete literature review, and the frameworks and models. But we may not find another time in history when so many consensus activities are happening where we can make our measurements. In the first year, we also began to figure out what data to measure, how to measure them, and how to interact with the consensus groups.

Because of the unexpected opportunity in test sites and consensus groups, we remoriented the phasing of our research effort areas so we could work on the test sites and gather data in parallel with developing new frameworks and models. Late in the second grant year, we spent more time developing frameworks and models. These efforts were postponed in the later part of year one because of unusual opportunities in Consensus Process Studies mentioned earlier. We completed the preliminary literature search and started the discipline-oriented literature search late in the first grant year. We've contilnued to work on the discipline-oriented literatura search in the second grant year and are developing a paradigm for consensus. Based on this paradigm, we began a paradigm-oriented literature search late in the second grant year and plan to continue this effort into the third grant year.

The research emphasis originally planned for the second grant year and the direction for the out-years is shown in Figure 2. The figure is included in the proposal and the first-year performance report. From this figure, the six effort areas and the three research domains we' 11 use for reporting are listed below. The parentheses indicate the type of research dominating the second grant year. For example, specific confirmatory research under consensus tools for methods means we make measurements and do statistical analyses on whether a given tool affects the consensus of a group. Global confirmatory research is an integrative effort, which combines the results of the specific confirmatory research efforts. Global confirmatory research occurs near the end of the grant effort when our world culminates in a guide to consensus. 
I. Fundamental Research

Theory and Concepts (exploratory)

(deeper 1iterature search in parallel with

frameworks and models and paradigm development,

inftial effort on consensus gathering expertise)

Consensus Tools for:

Methods (specific confirmatory)

(consensus measurements in consensus groups)

Rules (specific confirmatory)

(environmental varlables to control)

Frameworks and Models (exploratory)

(paradigm development related to original

taxonomies)

II. Consensus Processes

Test Sites (specific confirmatory)

(standardize and relate consensus group set-up)

Consensus Tools for:

Rules (specific confirmatoryr)

(relate environmental issues to the consensus process)

Consensus Methods (exploratory and specific

confirmatiory)

(apply methods in consensus groups)

Consensus Process (specific confirmatory and global confirmatory)

(apply tools from 11terature, develop new ones)

III. Information Systems

Test sites (specific confirmatory)

(relate information sources to Five-Year Waste

Plan execution)

Consensus Tools for...

Methods (specific confirmatory)

(establish information flows)

Within Domain I during the second grant year we brought in more students in a coordinated effort to focus on the tools for methods and rules for consensus. This enabled us to take better advantage of the consensus groups and meetings we set up under the other two domains. In Domain II and III, we set up the opportunity to gather more data and to do more studies than we'l.1 ever have enough personnel to interpret. The reason for the plethora of data was the unexpected and fortul:ous events relating to the presidential transition during the first grant year. Because we happened to be at this time in history, the whole consensus opportunity has become a windfall. One of our concerns in fundamental research was to figure out how to gather the data even if we don't have all the graduate students available to interpret the data. Another problem was: What data do you gather if there isn't one unfversallyaccepted definition of consensus? We've begun to define consensus and other related variables, and continued to refine our definitions during the end of the second grant year. 
Domain I for the second grant year was dedicated to 1) making sure we gathered the data while we had the opportunity with consensus groups, 2) guiding the students, theses, and dissertations to bear on some of these data, 3) moving the literature search to the paradigm level (including the discipline-orlented studies), 4) developing a paradigm (or conceptual mode1) of consensus, ij) defining independent variables of consensus, 6) developing relationships or categories for tools to get consensus and ldentifying or measuring consensus, 7) deveioping situation-specific methods to match up to certain situations (involving the five factors supporting consensus), 8) developing individual tools and/or methods to take advantage of targets of opportunity (early establishment of some consensus groups), and 9) presenting concepts and literature search, taxonomies, and a paradigm for critical review (papers and presentations).

Within Domain II during the second grant year we studied the consensus groups we established and developed criteria and alternatives for conducting meetings of and applying tools to the consensus groups. We continued to review the consensus activities relating to review of the Five-Year Waste Plan throughout year two of the grant.

Domain II for the second grant year was dedicated to 1) developing a checklist for defining and initiating a consensus group based on targets of opportunity, 2) establishing criteria for selection of members of consensus groups, 3) appiying tools and methods from the literature, 4) prototyping new tools and methods, 5) developing criteria for evaluating the consensus aspects of a meeting, 6) developing criteria and methods to evaluate effects of a given participant in the meeting on consensus, 7) developing criteria for choosing whether we want consensus from a particular meeting, 8) identifying instances of conflict in groups and evaluating effects of conflict and resolution, 9) developing methods to communicate results of consensus groups as part of the continuing presidential transition, 10) deriving methods for broadly sharing results of consensus groups and gaining feedback (extra-group consensus), 11) reviewing results of getting consensus in the Five-Year Waste Plan, 12) suggesting changes for the Five-Year Waste Plan update to enhance consensus; 13) evaluating how to implement the consensus aspects of Five-Year Waste Plan during the first year of the plan, 14) evaluating relationships between culture, plan, and other management tools, 15) applying methods in consensus groups, 16) identifying methods from the literature, and 17) presenting concepts and consensus group case studies and results for critical review (papers and presentations).

Within Domain III during the second grant year we evaluated the information people have avallable to them as they carry out the FiveYear Waste Plan both at headquarters and in the field. We addressed several questions. What is the right policy or philosophy for how all those information systems work together? Which information systems feed other information systems? Which information systems are similar enough to provide compatible information to a decision maker? Decision makers are going to be more involved in consensus type decisions than ever before. We focused on information systems that give all decision makers a lot of information so they can come together and make more coordinated or participative-type decisions. It's a different type of generation of 
information than DOE has dealt with before. We continued to consider the origins and flows of information into the Five-Year Waste Plan as we particlpated in the update process. This activity continued into the third grant year,

Domain III for the second grant year was dedicated to 1) scoping a conceptual approach for accessing consistent waste data from stakeholders throughout the DOE waste complex, 2) evaluating the information people have to carry out the Five-Year Waste Plan at headquarters and in the fleld--check for timely, accurate, and relevant data and information, 3) evaluating what should be done with information systems from the perspective of generating information for groups of managers making participative decisions, 4) evaluating information origins and flows into the Five-Year Waste Plan, 5) securing equipment and resources needed to set up the information gathering and communications laboratories, 6) Identifying what should be done with the information systems throughout the complex from an information systems perspective, 7) integrating waste information for EM to make consistent and global decisions, and 8) presenting concepts for critical review (papers and presentations).

Fundamental Research

\section{Background}

Consensus is an ideal group decision state, often strived for, but rarely achieved. Ideally, consensus assures complete understanding of the issues and options, total agreement on the best solution, and complete commitment and support for that solution (Gentry, 1982). Research concerning whether individuals or groups make better decisions isn't conclusive, but does suggest under certain conditions groups make better decisions. Our research emphasizes group decisions. Achieving consensus is dependent on a number of variables. Through our literature search we're building a paradigm of consensus which relates variables and is the framework for our research. We've identified and are studying a number of these varlables. For instance, we're researching the role of facilitators, experts, conflict, and information availability as they relate to consensus. We've begun preliminary measurements of the amount of information available to solve a problem to determine its effect on the degree to which consensus can be achieved.

Measuring consensus is difficult. The best way to characterize consensus is as a "hypothetical construct." We often presume consensus exists at some point when everyone's ideas have been combined into one complex whole. We may not be able to measure consensus directly. We may need to infer it. Some ways consensus has been measured are by degree of agreement, individual feelings about the probability of an event occurring, interaction patterns within a group, and individual preferences. 
Adding to this confusion is the fact that consensus refers to both 1) an ideal situation in groups (product) and 2) the ideal means to achieve group goals (process). Consensus in a small group setting can apply to a decision making process or the product of the process. Jay Hall, author, defines consensus as "a decision process for making full use of available resources and for resolving conflicts creatively." If we look at consensus as the ideal goal in groups instead of a process, we are still faced with different meanings. Definitions used in the literature for consensus range from the degree to which the most influential people in a group agree to perfect unanimity among all group members. Some people refer to consensus as a state of mind, a "we" feeling among the participants in a group, Irving Janis emphasizes the negative aspects of this in his book Groupthink. He defines groupthink as "... the desperate drive for consensus at any cost that suppresses dissent..."

Toward the end of the grant year we focused on designing instruments for measuring consensus in all situations. There are many decisions DOE personnel make every day which we considered measuring by other methods. Such decisions include prioritization, resource allocation, selection, : and compliance.

With the new push for culture change within DOE, there exists a need to help people work better together, be more open, and share information. We researched scenarios to be used with DOE groups comprised of people whose new job responsibilities require them to work together. Such a team building management tool would also be used with groups comprised of DOE employees and contractors who are being required to work closer together and share information.

While it's important to find ways to measure consensus so we know whether or not we've achleved it, our foremost task is to find how to achieve it in the first place. Our consensus research has focused heavily on techniques for achieving agreement. We've studied a number of group techniques for idea generation: brainstorming, brainwriting, idea writing, and forced relationship techniques.

Brainstorming is one of the most widely used group techniques for creating ideas. The theory is that verbally inputted ideas from group members will spark other ideas from other participants. These new ideas may in turn spark other ldeas from other group members, and so on. Alex Osborn, the originator of Brainstorming, calls this "organized ideation". The process should result in a large pool of ideas.

Brainwriting differs from Brainstorming because participants generate and submit ideas on $3 \times 5$ index cards instead of verbally inputting ideas. An advantage of this process over Brainstorming is group members are more likely to submit any idea simply because the source of the idea is unknown to the group.

Idea Writing is a more structured version of Brainstorming and works best with groups of four or five. Each participant writes three ideas separately on three index cards and passes the cards to the left. The next participant sorts the cards in any manner he or she wishes (e.g., importance, usefulness, simplicity) to help strulcture his or her 
thoughts on the problem. The participants add three more ideas to the cards they have and then pass them to the left. This process continues until each participant has written twelve ideas and sorted the last stack received.

Forced Relationship Techniques are structured techniques to aid in stimulating new ideas. Two or more ideas, objects, or methods are forced together to produce something new. Because of the structure of these teckniques, they generally result in a more focused list compared to unstructured techniques like Brainstorming or Brainwriting. Forced relationship techniques include transformation and attribute changing. Transformation is used to change an object or a process to make it more useful. Attribute changing breaks an object or process down into its characteristics and generates a list of possible ways to change the attribute.

We've also studied group techniques for problem cause identification: Pareto Ane jsis and Cause and Effect Analysis. Various techniques are employed to examine the cause of a problem.

Pareto Analysis delineates the causes of a problem and helps the group focus on the most important causes. It does this by using a pareto diagram. The pareto diagram consists of a combination bar graph and line graph. The $X$ axis of the pareto graph is typically the individual causes of the problem. The $Y$ axis is typically a measure of the problem (e.g., cost to the company). By viewing separate causes of the problem together, measured on a common scale, it's easy to see what causes are the greatest contributors to the problem.

Cause and Effect Analysis also incorporates a graphical method, cause and effect diagram, to show relationships between causes and the effect of those causes for a given process or problem. The effect of a problem is listed at the end of a long arrow. Then all causes of the effect are grouped by category and linked along the arrow. This produces a "fishbone-like" diagram. This diagram can be used to understand the relationships between causes of a problem and aid the users in identifying "attack areas" for problem resolution.

Our research effort has mainly focused on group techniques for idea generation and decision formulation. We've studied and tested a number of techniques for group decision making: Nominal Group Technique, Improved Nominal Group Technique, Delphi Technique, and "Generic" Interactive Group Decision Process.

Nominal Group Technique (NGT) is a structured group decision technique consisting of four steps: 1) individual silent generation (i.e., group members write their own ideas about the problem), 2) round-robin recording of ideas ( $1 . e$. , each group member, one at a time, presents one of his or her ideas to the group without discussion, 3) group discussion for the purposes of clarification and evaluation, and 4) individual voting on priorities through rank-order or rating procedure. "The group decision is the 'pooled' outcome of individual votes" (Van De Ven \& Delbecq, 1974). 
Improved Nominal Group Technique (INGT) adds several steps to NGT. First it uses Delphi's (see below for description of Delphi technique) method of inputting and review of ideas (through the mail) before the actual meeting takes place. Next, instead of inputting ideas for clarification and evaluation verbally (as in NGT), INGT has group members submit 1deas on $3 \times 5$ cards (maintaining group anonymity). Inputting ideas on $3 \times 5$ cards also allows more than one input at a time (reducing bottlenecks). Lastly, INGT provides for post voting, ranking discussion, and a second round of voting ranking if the group so desires.

Delphi Technique is a group decision process that provides for the systematic solicitation and collation of fudgments on a particular topic through a set of carefully designed sequential questionnaires interspersed with summarized information and feedback of opinions derived from earlier responses (Van De Ven \& Delbecq, 1974). Two iterations of questionnaires and summary reports are used. The first questionnaire is designed to obtain information on the topic or problem. It is distributed anonymously to participants through the mail. (Note: This is the "diverging" questionnaire). The participants generate their responses to the questionnaire and mail these back to the administrator of the process. The responses are summarized into a feedback report. This report, along with a second questionnaire designed to probe more deeply into the ideas developed in the first questionnaire, is sent back to the participants. (Note: This is the "converging" questionnaire). Participants independently [and anonymously] evaluate the feedback and respond to the second set of questions. Typically, participants will be asked to vote independently on priority ideas. Participants return their second responses to the administrator by mail. Generally, a final summary and feedback report is sent to all participants.

"Generic" Interactive Group Declsion Process is an interactive group decision process with a typical format that starts with a problem statement by the facilitator to the group. A group discussion for generating information and pooling judgments follows. The meeting concludes with a majority voting procedure on priorities, or a consensus decision.

We've researched group techniques for decision formulation, decision analysis and judgment evaluation. These techniques go beyond formulation of decision and analyze the result. These techniques are Consensus Mapping and Social Judgment Analysis.

Consensus Mapping group decision process assumes a task group has already (a) generated a list of ideas about a particular issue or problem under consideration, (b) clarified the meaning of those ideas, and (c) conducted a preliminary evaluation (i.e., ratings or prioritization) (Hart, et al, 1985). Therefore, Consensus Mapping would start where a process like NGT would finish. Consensus Mapping uses two to four task groups (usually five to nine members each).

Social Judgment Analysis (SJA) is based on Tolman and Brunswik's approach to cognition (Tolman, 1932; Brunswik, 1943; Tolman \& Brunswik, 1935) and includes: (a) placing a weight (i.e., a particular degree of 
importance) on a piece of information, (b) developing a "functional form", or a functional relation between each piece of information and the final judgment, and (c) using a particular method for integrating all dimensions of the problem (this is called "organization principle").

SJA uses "cognitive feedback" to help reduce disagreement and improve the quality of judginent. It allows participants to deal openly with conflict and/or disagreements in underlying judgment policies. They can see the exact locations of agreement and disagreement. Participants examine the weights and functional forms of other participants. Therefore, they can understand why another participant makes a particular judgment by looking at the importance the other participant places on each piece of information and the functional relation between each piece of information and the other participant's final judgment. These functional relationships are represented by individual "cognitive maps".

We examined a few group techniques incorporating conflict. These techniques for arriving at a decision include conflict as part of the process. They are: Dialectical Inquiry and Devil's Advocacy.

Dialectical Inquiry uses conflict among two teams to arrive at a quality decision. It develops two different recommendations, based on contrary assumptions, from the same data. The group divides into two teams, each team taking a side. The teams debate each recommendation to spell out the implications of each decision, revealing its underlying assumptions, and challenging (defending) those assumptions as effectively as possible. The assumptions that survive the scrutiny of the debate (along with new assumptions formed during the debate) are grouped and recommendations are formed by the group members. These final recommendations are the group's solution.

Devil's Advocacy also uses conflict to arrive at a quality decision, but it does it differently than Dialectical Inquiry. In this approach, a solid argument is developed for a reasonable recommendation. This recommendation is then subjected to a formal critique that attempts to show why the recommendation should not be adopted. Through repeated criticism and revision, a mutually acceptable recommendation is formed.

So the difference between Dialectical Inquiry and Devil's Advocacy is that Dialectical Inquiry starts with two recommendations and employs a debate to arrive at a mutually exclusive decision, while Devil's Advocacy uses one recommendation and employs criticism and modification to arrive at a mutually exclusive decision.

We've studied the few documented group techniques for gaining consensus: Hall's Consensus Guidelines and Social Judgment Analysis. We'11 continue to study and search for such techniques and design our own group technique for gaining consensus based on previous work.

Hall's Consensus Guidelines provides rules to follow for gaining consensus. Hall (1971) noticed through experimentation that groups with formal training perform consistently better than groups without. He summarized the behaviors of the most effective groups and translated 
them into a list of guidelines for consensus gaining. The guidelines are:

1. Avoid arguing for your own rankings. Present your position as lucidly and logically as possible, but listen to the other members' reactions and consider them carefully before you press your point.

2. Do not assume that someone musc lose when the discussion reaches a stalemate. Instead, look for the next-most-acceptable alternative for all parties.

3. Do not change your mind simply to avold conflict and to reach agreement and harmony. When agreement seems to come too quickly and easily, be suspicious. Explore the reasons and be sure everyone accepts the solution for basically similar or complementary reasons. Yield only to positions that have objective and logically sound foundations.

4. Avoid conflict-reducing techniques such as majority vote, averages, coin flips, and bargaining. When a dissenting member finally agrees, don't feel that he or she must be rewarded by having his or her own way on some later point.

5. Differences of opinion are natural and expected. Seek them out and try to involve everyone in the decision process.

Disagreements can help the group's decision because with a wide range of information and opinions, there is a greater chance that the group will hit upon more adequate solutions.

Social Judgment Analysis, which we've already described, can also be included as a consensus gaining technique.

The above discussion illustrates some of the state-of-the-art techniques available for decision making in general and the consensus process in particular. But a "tool kit" of avallable techniques does not provide us a sufficient understanding of consensus. Nor does it enable us to validly measure it, or know when we've achieved it. Our research is designed to address these issues.

During the last part of the second grant year, our fundamental research accelerated. We made progress in our efforts to understand, conceptualize, measure, and influence consensus. The following paragraphs detail our progress.

\section{Accomplishments}

For fundamental research, we worked on thirteen studies during the second grant year. During the second grant year we added three new studies in fundamental research. The three new studies are: 1) consensus teshniques, 2) consensus facilitators, and 3) consensus scenario generation. 
In the Consensus Library, we've begun to use MSLSearch, our on-1ine computerized reference system. MSLSearch performs two major functions. First, bibliographic information and locator descriptions are entered to allow easy retrieval from MSL's library. Second, we can enter article summaries which include key research findings, variables, key words, hypotheses, definitions, and conclusions from each reference. We use the summaries for sorting and organizing reference material to aid the graduate students, MSL and adjunct researchers, and attached research faculty. The summaries will help in preparing articles, theses, dissertations, bibliographies, conference papers, other scholarly and professional presentations, and in preparing materials for use in developing and testing consensus tools within the test sites.

We've identified 257 articles we want to review and have reviewed 186 of these. We now have the results of an electronic search conducted at the Library of Congress, giving us access to some 5,000 titles. We've begun to identify articles we're interested in reviewing and are identifying and categorizing variables. At present we've identified 13 disciplines around which we'11 organize the literature search. They are:

communications, computer science, economics, education, group behavior, human factors, management, management science, organizational behavior, political science, psychology, sociology, and social psychology. We've identified 76 variables and have placed them into preliminary

categories. We've also developed a preliminary paradigm of consensus we'll use to organize our research.

As our research progresses, the dissemination purposes of the library and MSLSearch will remain the same, but the collection purpose will shift from general to specific in response to the more particular needs of filling ifterature gaps to support more precisely defined ongoing research activities. For example we've already begun an extensive literature search on group consensus development and management. This has involved an inventory of several thousand documents to be reviewed, and where appropriate, abstracted.

A second major emphasis was on the development of new theories and concepts to support our research of consensus. Though we already began this effort in our preliminary research, emphasis on this area will grow as results indicate inadequacies in current theories and concepts.

In Consensus Process Studies, we studied intra-group, inter-group, and extra-group consensus (the three types of consensus we've defined) by observing working groups under varying circumstances. For instance we researched intra-group consensus by forming an autonomous work group. To investigate the need for facilitation to reach consensus, the group met for six months without a facilitator and six months with an outside facilitator. We examined ways to measure consensus in non-ranked situations in small group interaction. We observed and recorded groupprocess data at a number of DOE-initiated meetings so we could analyze this data in terms of consensus-related variables.

In Consensus Paradigm Development, we identified relationships among the variables of consensus found in the discipline-oriented literature search. Given the various definitions from the disciplines, we 
constructed a first draft of the paradigm or model. We looked at variations of components of the paradigm to determine practical applications. We used our initial paradigm to structure the remainder of the literature search.

In Organizational statics and Dynamics, we used an exercise called the Lost on the Moon test (developed by Jay Hall) to measure the strength of consensus within a group. We defined the strength of consensus as the extent to which the individual rankings done after the group exercise correlated with the consensus ranking done by the group. We recorded the group discussion and conducted an interaction analysis as a separate measure of the strength of the consensus. Starting with methods proposed from the literature, we continued to determine empirically which methods are best for achleving the strongest consensus.

In the Consensus Academic Plan, we scoped a plan for approaching our research activities both within each of the three research domains and across other domains. Based on the literature search, this academic plan is more detailed than the plan in the proposal. We must continuously update the plan to accommodate targets of opportunity as well as new findings. The plan is crucial for maintaining an integrative understanding of consensus as we study consensus and outcome processes across the three domains.

In Consensus Case Study Research, we studied and compared the specific historical case studies involving consensus processes we selected in the first grant year. We examined key decisions made by consensus in four areas: foreign policy/peace negotiations, emergency relief, Presidential advisory groups ("kitchen cabinets"), and labor/management negotiations. We identified elements present in the successful decisions, as well as those absent in unsuccessful decisions. Through this process we identified essential elements of a successful consensus decision. This study will be completed during the next grant year.

In Consensus Gathering Systems, we continued to develop a system that accepts the ideas of group members individually, understands areas of agreement among the ideas, works on compromise and support, and identifies the degree and breadth of consensus. This consensusgathering system has the ability to understand what the users say, extract the users' ideas from what they say, identify similarities and differences between the ideas, and set-up an hypothesis as a consensus among the group. Such a system must be grounded in a solid understanding of the rules of consensus and the consensus process.

In Consensus Group Dynamics, we looked at several variables related to group commitment. We reviewed the relevant iiterature and identified five independent variables affecting consensus of a group: commitment, homogeneity of group, conflict, leadership, and power. We also expanded a compilation of instruments that others have used to measure consensus. related variables.

In Consensus Techniques, we studied a number of different group decision making techniques. For example, Nominal Group Technique, Delphi Technique, Social Judgment Analysis, and Brainstorming. Based on our 
research of other techniques, we developed our own technique for reaching consensus. We studied other techniques and, when applicable, update our technique as needed. We also looked at matching techniques to different applications based on the components of the consensus paradigm. Then, we worked on the development of a management tool to help a facilitator select the best technique for reaching consensus.

In Consensus Facilitators, we studied the role of the group facilitator in assisting group members through their interactions. Factors such as the facilitator's knowledge of the group dynamics and the personality traits of group members were the focus of our research. We plan to use the results of these studies to dictate what knowledge and tools facilitators-in-training need to learn to more effectively and efficiently facilitate group meetings.

In Consensus Scenario Generation, we analyzed information to develop DOE applicable scenarios for problem solving requiring a consensus decision. Using supporting information from the literature search, our qualitative data gathered from observing consensus meetings such as Consensus Group 2 , and our experience testing from the Lost on the Moon exercise (under the study Organizational Statics and Dynamics), we developed a clear definition of the problem and some form of choice for the solution. In the third grant year we'11 choose one scenario and develop it into a management tool for team building.

In Information Avallability, we conducted an experiment designed to examine the effects of information avallability on the group consensus process. We were particularly interested in process gains and losses resulting from social interaction and uncertainty based on the information available to the group as a whole. In our experiment we had eight small groups execute the Lost on the Moon exercise with each group receiving one of three levels of information about the Moon or the problem situation. Each individual generated a solution to the problem then each group generated a solution. To measure group consensus, we measured decision quality of both individual and group solutions. Group decision quality scores were better than the average of individual decision quality scores in all cases and groups were highly committed to their consensus ranking regardless of their assigned information level.

Presentations and Papers are included in Volume II, Papers, Presentations, and Conferences.

Consensus Process Studies

\section{Background}

The magnitude and impact of the U.S. Department of Energy's (DOE) environmental restoration and waste management program requires a drastic change in DOE's culture to include the participation of all levels of government, public forum representatives, and the public. This change has provided us with a wealth of opportunities to observe consensus processes as groups meet and try to come to agreement on 
various issues. Much of our research has focused on capttalizing on these targets of opportunity.

Consensus Group 2, now called the State and Tribal Government Working Group. (STGWG), is an on-going group that meets approximately four times a year. Members review drafts of the future Five-Year Plan and other issues the DOE brings before them. Throughout this precedent setting process, MSL has taken the independent role of observer/facilitator. In addition to the responsibility for setting up and carrying out the meetings, we've acted in the role of coordinator and troubleshooter. We've strived to take a diverse group of individuals and get them to work together as a group attempting to help the DOE solve environmental management and cleanup problems.

Intial members of STGWG were gubernatorial representatives from nine states with major DOE facilities in them: Colorado, Idaho, Kentucky, Nevada, New Mexico, Ohio, South Carolina, Tennessee, and Washington. Also, members of this group included two Indian nations with major DOE facilities on their reservations (Yakima and Shoshone/Bannock); representatives from the National Governors Association; National Council of State Legislatures; and the National Association of Attorneys' General. Secretary Watkins guided us in choosing these participants because of their profound interest in DOE environmental restoration and cleanup activities.

The relationship between the STGWG and the DOE has been successful and highly beneficial to both. The DOE has made a significant commitment to the STGWG and has tried to incorporate changes it suggests in the FiveYear Plan. Throughout the meetings, Secretary Watkins has remained in close contact with DOE personnel so he can provide real-time response to STGWG comments. The first meeting in June 1989 resulted in two major changes in DOE policy which were written into the Five-Year Plan. The first was a time frame for the cleanup of DOE installations. The DOE also agreed to incorporate into the Plan a clear acknowledgement of state and tribal authority in the cleanup process. At the second meeting in July, DOE expended this acknowledgement and agreed to negotiate with states and Indian nations for full funding of all regulatory activities directly related to DOE sites and facilities.

As the meetings continued we learned more about the processes, participants, and the product. As we applied lessons learned, the meetings didn't take as long and the product was of a higher quality. The STGWG was now concerned more with policy issues. The product became a list of recommendations/comments about the Plan which the STGWG would like to see addrisssed.

As a result of the STGWG meetings in October and April, DOE agreed to the following: 1) to develop subsequent Five-Year Plans consistent with a 30-year plan commitment, 2) to seek legislation from Congress establishing a national commitment to the 30-year goal, 3) to drop all calls for uniform environmental regulatory national standards, 4) to set up the Public Interest Review Group to increase public participation, and 5) to expand the STGWG to include all states with a DOE facility, no matter how small. 
Toward the end of 1989 , our role expanded to include the responsibility of coordinator as well as observer of the working group meetings. It was decided that our work would best fit under an alternate funding source beginning January 1990. Therefore, our role under the grant was limited to observing the STGWG meetings to gather data in support of our consensus process studies. Therefore, any data gathered would be reported within Domain I under Consensus Process Studies.

Another opportunity to observe consensus processes was with our involvement as coordinator and facilitator of Environmental Restoration Program managers' meetings. The purpose of these meetings was to provide a forum for DOE Envirunmental Restoration Program Managers for DOE Headquarters and 1 ts various field offices to: 1) exchange information and share experiences and lessons learned concerning environmental restoration management; 2) work together on common environmental restoration management problems; and 3 ) discuss specific items on the environmental restoration meeting agenda to elicit thoughts and questions. The meetings focused on programmatic discussions as opposed to discussion of technical problems.

The concept of holding regular Environmental Restoration Program managers' coordination meetings was suggested at a management coordination meeting in Denver. It was decided to hold these meetings once each quarter at different operations office locations. When possible, the meetings included tours and briefings at site locations of the environmental restoration projects.

Each meeting included topics suggested by MSL, DOE Headquarters, and field office participants. MSL used these topics to develop the final agenda and arrange for speakers. MSL, with DOE Headquarters, also facilitated each meeting. Following the meeting, MSL worked with DOE Headquarters to prepare a follow-up report on the meeting. This report contained a synopsis of the issues discussed and what consensus if any was reached, as well as any action items that needed to be taken. It also suggested possible changes in the structure of future meetings such as changing the format or group participation. At the end of this report, MSL suggested issues for discussion at the next meeting based on ideas for topics solicited from participants in follow-up discussions.

EM (Office of Environmental Restoration and Waste Management)

information briefs were a useful tool to communicate the new office of Environmental Restoration and Waste Management's program activities to a varlety of audiences. The audience included congressional staffers; people new to the EM program; and members of the general public with a vested interest in the EM program.

These briefs were modular in design so they can be easily tailored to meet the intended audience and purpose of the presentation. These briefs were useful in effectively communicating EM's major prograin activities to a wide range of audiences during a time when EM is quickly and vastly growing.

Besides the design and developinent of information briefs to effectively communicate EM's major programs, MSL was tasked in June 1990 to 
research, design, and develop a booklet about EM's major program activities. Once again, the audience for the booklet was congressional staffers, people new to the EM Program, and members of the general public with a vested interest in EM's Program. The purpose of the booklet was to be a tool people could use to learn about EM's major activities. It would be used as handouts at congressional hearings, public meetings on DOE policy, and conferences related to DOE's activities. Knowing the audience and purpose of the document, MSL designed the document to contain a minimum of words with a maximum of photos and graphios to describe EM's major activities.

lany regulations, which are at times overlapping and conflicting, govern the land disposal of waste. The development of a consensus tool was needed to guide EM, the Office of Environment, Safety, and Health (EH) anc others in understanding these regulations and their applications. MSL was asked to work with EM and EH to research land disposal guidalines and develop a consensus tool for effectively communicating these regulations.

We had another opportunity to observe consensus processes when representatives of the U.S. Department of Energy (DOE) met 1teratively with over twenty local government officials from clties and counties around the country. The DOE officials were from the Transportation Management Division and the local officlals were primarily senior and mid-level hanagers with interests in transportation and emergency management. MSL, in coordination with the Energy Task Force Management Corporation (now called the Urban Energy \& Transportation Corporation), designed and facilitated the meetings with these goals:

1. Share information that local government officlals can apply to their own communities;

2. Exchange experiences and ideas applicable to other emergency management programs; and

3. Identify areas of productive action for DOE and local government to address issues of mutual concern.

The highlight of the last meeting was a progran planning exercise. The participants, playing; the roles of federal managers in DOE, developed programs to address the concerns of local governments on transportation of hazardous and nuclear materials, and emergency prepdredness related to incidents involving shipments of those materials.

The program planning exercise used meeting techniques designed to develop ideas for a Depaltment of Energy program plan to address local governmental concerns aboldt emergency preparedness for nuclear materials transportation incidents. Participants were asked to play the role of a staff member for a DOE administrator, to review a report identifying local government concerns (the "report" used was based on the actual product of an earlier workshop), and to develop a program plan for FY90 and subsequent years to address those concerns. 
We designed a structured process to accomplish our objective and maximize our limited time. The process was interactive, collectively bullding a consensus plan based on both individual partiolpation and team thinking. The exerolse placed the particlpants in the role of a federal manager faced with the difficult job of trying to satisfy transportation concerns while staying within organizational constraints. We hoped the result of our participative exercise would be two-fold: first, to generate a program of initiatives to address local concerns (and suggestions for their implementation); and second, to foster a better mutual appreclation of the problems facing all of us in developing such a program.

To do this we designed an exercise with three main parts. First we divided into teams for the purpose of designing programs to meet our objective. For the second part we reconvened to have team leaders report back to the larger group on the initlatives and implementing steps developed by each team. And for the third part we discussed the varfous aspects of each plan and achleved consensus on a composite proyram plan that might serve as a model for continued cooperation between DOE and local governments.

Since Management Systems Laboratories is now supporting DOE's institutional interactions with local transportation officials through a separate cooperative agreement, we decided to discontinue the work.

\section{Accomplishments}

For consensus process studies, we worked on elghteen studies during the second grant year and added five new ones. The five new studies, all short-term, are 1) site specific plans (iummunity relations), 2) FiveYear Waste Plan update, 3) plan update process: ADS system, 4) waste operations emergency preparedness, and 5) the EM Primer. We completed twelve studies during the second grant year. Four of these completed studies were moved to other funding sources. The four studies are: 1) consensus group 2 (institutional), 2) consensus group 3 (interagency), 3) consensus group 1 (EMRG), and 4) Nuclear Waste Transportation.

In Manogement Analysis and Networking, we analyzed DOE's culture which traditionally was an inward looking, secretive, technically speaking organization. DOE's desire was to become an open armed, outward reaching, broadly speaking organization in environmental, sociological, psychological, and technical terms. DOE wanted to speak to the larger public to derive consensus for its activities and move its old culture to a new, consensus orlented culture. We were involved with DOE headquarters, fleld sites, contractors, and off-site participants to observe the changes taking place. To assist DOE with identifying the organizational structure it needed, MSL visited field sites to study how changes were impacting the present organizational structure. We brought together organizational experts who worked for DOE for years and understood its old culture and documented their ldeas for an organizational design more suited to the new culture.

In Consensus Meetings, we continued to encourage consensus between headquarters and fleld sites by setting up and studying various 
consensus groups during the year. These meetings involved representatives of the U.S. Department of Energy and over twenty local government officials from around the country. We tried out different processes on different consensus groups as they met at different times and on different subjects. For instance, we found meetings were more successful if: 1) workbooks were provided; 2) the participants had thorough knowledge of the issues; and 3 ) we employed a proper mix of informal and formal procedures. We made observations leading to implications on new tools and methods and on possible sensitivity of potential variables.

In the past, Environmental Restoration Proglam managers' meetings occurred quarterly. However, this past year we only had one meeting in May 1990 in Santa Fe, New Mexico, due to federal budget cuts. During the meeting, MSL worked with the host, the Albuquerque Operations Office, to develop issue topics for discussion. Many of these topics were derived from discussions with regular participants of the meetings. MSL also designed the format of the meeting and facilitated it with DOE Headquarters.

Ir Consensus Group 2 (Institutional), we observed the consensus processes of the State and Tribal Government Working Group (STGWG). In addition, we set up several meetings and served as the facilitator, coordinator, and troubleshooter. We tested different procedures to bring this diverse group of Individuals together as a group working to solve environmental management and cleanup problems. We tried to operate the group to give the stakeholders enough freedom and flexiblilty so they didn't feel constrained while we got the full benefit of their views. Our role developed into one of an observer and it was decided our work best fit under another funding source. However, data gathering would continue under Domain I, Consensus Process Studies.

In Consensus Group 3 (Interagency), we didn't have the opportunity to bring together federal agencles like EPA (including all eight regions), $O M B$, and others as planned. Such agencies represent an entirely different type of stakeholder from the other consensus groups we've observed. Due to the difficulty of bringing these agencies together, we're looking for independent opportunities to observe these types of groups. Our research shows achleving consensus in this type of situation would be most difficult, therefore; this study has been completed. Any future attempts af observing for data would be reported under Domain I.

In EM Command Briefs (old Transition Management), we observed and studied Secretary Watkins' culture transition in DOE. The culture transition continued to take place. MSL spent considerable time studying and researching culture transition in other organizations. For instance, AT\&T's culture transition has been a massive and dramatic as DOE's. AT\&T is a monopoly and much like a government organization. It was secure, emphasized quality and service, and considered cost later. Now AT\&T must be a competitive, cost-oriented organization which removed security from its employees. AT\&T managed its cultural transition by defining and symbolizing 1 ts new culture and by drawing pictures of a "shared values pyramid." AT\&T instituted a huge promotional campaign to 
communicate the culture and get people's commltment to the culture. We observed the approach DOE used to institute its new culture and compared the process and results to those of AT\&T and other organizations who have taken a proactive approach to changing culture.

We worked with the transition, in general, and the culture transition, in particular. We emphasized how to manage the transition. We researched what sources of information should be presented and whether or not that information should be issue-oriented or status-oriented. We studied and tried to determine who should play what roles, what kinds of information were appropriate, and what was the purpose of the information. We researchod ways to pull together a new organization and studied the effects of growth on an organization as it relates to consensus. Our research resulted in a ten-step strategy for managing culture change. We researched, designed, and developed modular briefings which described EM's major program activities by operations office. These briefs were used by $E M$ to effectively communicate its programs to several audiences including program managers new to EM and members of the general public with a vested interest in EM's programs.

In Continuous Information Transfer (Video), we experimented on developing and cataloging systems for libraries for information portrayal tools. We studied ways to portray information so you don't necessarily have to have a person present to have a continuous flow of information pre-designed for that purpose.

In Discrete Information Transfer (Modules), we studied ways to put together a presentation in discrete packages so you can address the specific needs of the audience. Instead of a continuous presentation of information, you have discrete pieces where you can select and choose what plece fits the audience. Our rule is audience plus purpose equals design. We also researched ways of presenting information on slides and view graphs to maximize audience interest. This study has been completed.

In Consensus Group 1 (EMRG), we ceased to be involved in facilitation of the group. This was turned over to the Waste Policy Institute. This study has been completed.

In Nuclear Waste Transportation, we observed consensus processes between DOE and local governments. Transportation of nuclear waste frequently involves the cooperative efforts of local, state, and federal officials. The wide geographical location and diverse interests of stakeholders increased the complexity of group meetings. We brought together and facilitated such a group at several workshops and a national meeting. We collected data for our consensus research. This study has been closed and moved to another funding source.

In Regulatory Consensus Research (Land Disposal Restrictions), we research the guidelines by DOE's Office of Environment, Safety, and Health (EH) and the regulations determined by the Environmental Protection Agency. We studied land disposal restrictions to guide our efforts to design a consensus tool for Environmental Restoration and Waste Management and others to use and apply to disposal of waste. We 
worked with EM and DOE's Office of Environment, Safety, and Health (EH) to study and disposal restrictions and guide our development of a tool for EM, EH, and others to use and apply to waste disposal.

In Site Specific Plans (Community Relations), we examined information from the field as it related to an overall top-down plan. We looked at how to involve local participation in five-year planning. DOE's culture began to change to a more open culture which encouraged community participation and involvement in policy and planning. As communities were given opportunities to influence the Flve-Year Waste Plan, tield sites were provided with guidance for future communication and interaction with their communities: We reviewed the plans from each DOE site to get an idea of how it conformed with the Five-Year Plan. The Site Specific Plans evaluated and helped implement the Five-Year Plan in the field. This study has been completed.

In Waste Operations Emergency Preparedness, we developed a Duty Officer Handbook for the Office of Environmental Restoration and Waste Management in accordance with DOE Order 5000.3A, which states that all headquarter Program offices with emergency responsibilities must have duty officer procedures to ensure that the office has a 24 -hour point of contact. The handbook described the headquarter Emergency Operations Center (EOC) and how the duty officer should interact with the headquarter EOC coordinators. The procedures outline the duty officer schedule, shift changes, and initial emeribency notification responsibilities. This task has been completed.

In the EM Primer Study, we researched, designed, and developed various drafts for DOE Headquarter's review of the EM Booklet entitled, "Environmental Restoration and Waste Management Program - An Introduction." The booklet was designed to provide an overview of EM's program which would assist the general public as well as EM's staff.

In Five-Year Waste Plan Update, we continued to follow up and apply lessons learned on the consensus activities generated by Five-Year Waste Plan. The Five-Year Waste Plan is the vehicle Secretary Watkins will use to initiate the change in DOE's culture. We spent a vast amount of our energies in the first grant year participating in the shape and form of that plan. Its form is designed to promote understanding so people of different backgrounds and different desires and agenda can do what they need to do with the plan. MSL's influence produced sore of a plan as opposed to a status report. We don't belleve consensus would be worth stidying if the plan was a status report. Instead the Five-Year Waste Plan addresses the issues people need to come to consensus on. This study has been completed:

In Plan Update Process: ADS System, we made suggestions for improvements to the Five-Year Waste Plan as a result of our research and observations on the construction of the plan. Our waste information data base containing technical waste information, programmatic waste information, and transportation waste information can be used to help streamline the Five-Year Waste Plan. This study has been completed. 
Presentations and Papers are included in Volume II, Papers, Presentations, and Conferences.

In Networking for Consensus (UME), we continued setting up bridges and networks, making contacts, having discussions and interviewing people, providing information to those people, and convincing those people to participate in the new DOE culture. Our consensus management research activities were based on the consensus elements identified from the historical case studies (under Consensus Case Study Research). We developed an early consensus management laboratory that validated those consensus elements by using a volunteer group of Virginia Tech employees to assess the validity of consensus elements. We conducted research of a variety of group facilitation techniques to be employed in the group consensus process. This also included a profile of characteristics that should be evident in a group facilitator. We also assisted in the development of a series of consensus group evaluation surveys.

Information Systems Studies

\section{Background}

DOE managers use information to make decisions setting policy and strategy. They must make these decisions based on intermediate information--unorganized and informal information .. that is not always complete. There are two main reasons for this. First, it's sometimes difficult for DOE managers with different preferences to agree on the information's content. Decisions are then made based on incomplete information since managers have not reached consensus on what's needed. Second, because of the lengthy concurrence chain, decisions must often be made before information can pass through a time-consuming approval process. In both cases, improved management of intermediate information through automation can lead to more effective information sharing which leads to more effective decision making. This is because automation 1 ) adds meaningful structure to otherwise, informal information, 2) directs the flow of intermediate information to the appropriate DOE manager for review and approval, and 3 ) reduces the time involved in concurrence activities.

An example of improved management through automation, is the PIP Concurrence System which automated the process required to concur on and produce a DOE planning document' the Office of Environmental Restoration and Waste Management (EM) Program Implementation Plan (PIP). The PIP Concurrence System can be accessed through the Waste Information Network (WIN) System. The PIP had to be concurred on by various DOE managers at the Field and Headquarters (HQ) level.

The 1987 PIP was created, reviewed, and concurred on through the use of time-consuming communication tools (mail, phone, facsimile, etc.) without a defined concurrence process. Structuring and defining the automated concurrence process dramatically reduced the time and overhead cost required for the concurrence process on the 1988 PIP. However, 
lessons can be learned from the PIP Concurrence System which can be applied to other documents requiring concurrence.

We believe managers at different organizational levels may need different data and information about events or actlvities for their decision making. If so, different managers may go to the same formal data stores and not find what they need. What are these differences in data and information needs? If designers of large computer systems don't know the answers to these questions, they can't meet managers' needs. Since hierarchical organizations have overlapping domains of responsibility, events or activities performed at the bottom of the organization are directly managed at the bottom of the organization and indirectly managed up through the organizational hierarchy. How does such a structure influence design of information systems?

The design of information systems is still very much an art. We design information systems by looking at the characteristics of information, decisions, and the manager. For information system design to move from an art to a science, we need a detailed understanding of data, information, and the process by which data become information. Information systems are falling because system designers and operators don't have enough detalled understanding of data, information, and the data-to-information process to satisfy managers information needs.

There currently are no comprehensive frameworks which define data and information in enough detail to describe the data-to-information process. Worse, there's confusion about the terms "data" and "Information", and the two terms are often used interchangeably according to Appleton (1986). Upon close examination of the literature, we found definitions and relationships that each serve to describe a portion of the data-to-information process. By assembling these definitions and relationships, we've developed a framework to describe data, information, and their relationships. We're working on defining data and information with enough detall to describe differences in data and information needs at different organizational levels.

Drucker (1966) says a manager converts information to action through decisions. Most managers rely on information tools to provide data and information. Together, the manager and the tools perform the data-toinformation process. The point in the data-to-information process at which the tools stop and the manager continues the process is the point at which portrayal occurs. Portrayal can be data, quantitative or qualitative information. We define four portrayal formats: tables, graphs, checklists, and narratives. The purpose of portrayal is to convey the results of the data-to-information process to the manager at the appropriate step in this process. The appropriate step should be the point at which the manager is capable of completing the process without further help from the tools.

Portrayal takes place at different steps for different managers and different situations. Managers can perform the entire data-toinformation process, as when a manager walks around his or her domain to observe the operations first-hand. Managers can also perform little of the data-to-information process, as when a manager hires a consultant to provide qualitative information for decision making. In this case, the 
consultant is acting as an information tool for the manager. The portrayal interface between the manager and the tools depends on the manager's experience and knowledge, organizational level, personality type, and the complexity of the problem to be solved.

Information systems should be built and operated with an understanding of the data-to-information process they support. The data-to-

information process serves as a guide to providing the right data and information to managers by showing the data components, data, and information types, and how data become information. Further, the portrayal interface between the information tools and the manager describes the right way to provide data and information to the manager. If the tools complete more steps than needed by the manager, the information tools are inefficient, and the manager loses the ability to use his or her personal preferences in the data-to-information process. If the information tools complete fewer steps in the process than the manager needs, the portrayal will be incomplete, and the manager will be unable to produce qualitative information in a timely manner. The datato-information process points the way to the effectiveriess of the information tools (getting the right data and information to the manager) while the correct portrayal interface points toward the efficiency of the information tools (providing data and information the right way).

For effective and efficient transfer of information we must better understand organizational levels. Oxganizational levels are defined as levels of managerial activity or decision making. Managers are judged to be at the level of managerial activity or decision making they primarily perform, although all managers may perform some of every activity and decision. Parsons (1960) proposed three levels of decision making in an organization: institutional, managerial, and operational. Institutional decisions are performed primarily at the top of the organization and set broad goals and policy. Managerial decisions are at the middle of the organization and direct the activities of the organization and coordinate tasks. Operational decisions normally take place at the bottom of the organization and involve performing the activities of the organization.

Similar to Parson's framework, Anthony (1965) developed a taxonomy for managerial activity. Managerial activity is broken into three groups: strategic planning, managerial control, and opexational control. Managers performing strategic planning set policy, organizational objectives, and determine resources to be applied to attain these objectives. Managers performing management control assure resources are obtained and used wisely in the performance of the organization's objectives. Managers performing operational control are responsible for carrying out specific tasks.

Defining endeavor as any serious determined effort toward a purpose, Kurstedt (1990) proposes four endeavors based on the Anthony framework: strategic, tactical, operational, and clerical. Strategic endeavors are those where global efforts are aimed in a general direction using qualitative measures. Tactical endeavors represent wide efforts directed toward a tangible result using quantitative standards. Operational endeavors involve limited efforts focused on a fixed outcome 
using restricted methods. Clerical endeavors consist of local efforts constrained to explicit tasks. Three of these endeavors (strategic, tactical, and operational) are supervisory activities, matching Parsons' and Anthony's three levels of managerial activity. Clerical endeavors are not supervisory, although they are managerial activities. Managers at any organizational level may perform all endeavors, although one predominates if the manager is performing his/her job correctly.

Kurstedt provides operational characteristics for each endeavor in Figure 5. In these operational characteristics the terms "problem" and "opportunity" mean essentially the same thing. By comparing these characteristics against any endeavor we can determine if the endeavor is strategic, tactical, operational, or clerical. 


\begin{tabular}{|c|c|c|c|c|}
\hline $\begin{array}{l}\text { OPERATIONAL } \\
\text { CRITERLA }\end{array}$ & $\begin{array}{l}\text { STRATEGIC } \\
\text { ENDEAVORS }\end{array}$ & $\begin{array}{l}\text { TACTICAL } \\
\text { ENDEAVORS }\end{array}$ & $\begin{array}{l}\text { OPERATIONAL } \\
\text { ENDEAVORS }\end{array}$ & $\begin{array}{l}\text { CLERICAL } \\
\text { ENDEAVORS }\end{array}$ \\
\hline $\begin{array}{l}\text { WHAT } \\
\text { THEYDO }\end{array}$ & $\begin{array}{l}\text { Decide What } \\
\text { Problems } \\
\text { to Solve }\end{array}$ & $\begin{array}{l}\text { Decide What } \\
\text { Resources } \\
\text { to Use }\end{array}$ & $\begin{array}{l}\text { Decide How } \\
\text { to Solve } \\
\text { the Problem }\end{array}$ & $\begin{array}{c}\text { Solve } \\
\text { the Problem }\end{array}$ \\
\hline $\begin{array}{l}\text { WHAT THEY } \\
\text { MANAGE }\end{array}$ & Outcomes & Outputs & $\begin{array}{c}\text { Transformation } \\
\text { Path }\end{array}$ & $\begin{array}{l}\text { Execution } \\
\text { of Steps }\end{array}$ \\
\hline $\begin{array}{c}\text { WHAT IS } \\
\text { ACCOMPLISHED }\end{array}$ & Goals & Objectives & Missions & Jobs \\
\hline $\begin{array}{l}\text { SETS } \\
\text { PRIORITIES } \\
\text { FOR }\end{array}$ & $\begin{array}{l}\text { Outcomes and } \\
\text { Implied Tasks }\end{array}$ & $\begin{array}{l}\text { Allocating } \\
\text { Resources }\end{array}$ & Steps & $\begin{array}{l}\text { NONE } \\
\text { (They Monitor } \\
\text { and Implement } \\
\text { Prionties) }\end{array}$ \\
\hline $\begin{array}{l}\text { TYPE OF } \\
\text { EFFORT }\end{array}$ & Pursuits & Actimities & Tasks & Actions \\
\hline $\begin{array}{c}\text { FORMULATION } \\
\text { TOOL OR } \\
\text { GUIDANCE }\end{array}$ & Policies & Plans & Procedures & Inseructions \\
\hline $\begin{array}{l}\text { EVALUATION OF } \\
\text { FORMULATION } \\
\text { TOOL }\end{array}$ & $\begin{array}{l}\text { Relationships } \\
\text { of Plans }\end{array}$ & $\begin{array}{l}\text { Framework for } \\
\text { Procedures }\end{array}$ & $\begin{array}{l}\text { Value of } \\
\text { Instruction }\end{array}$ & $\begin{array}{l}\text { Yes/No } \\
\text { on Sieps }\end{array}$ \\
\hline $\begin{array}{l}\text { FORCING } \\
\text { FUNCTIONS }\end{array}$ & $\begin{array}{l}\text { Internal } \\
\text { and External } \\
\text { Organizational } \\
\text { Emvironment }\end{array}$ & $\begin{array}{l}\text { Chosen } \\
\text { Outcomes } \\
\text { Goale and } \\
\text { Implied Tasts }\end{array}$ & $\begin{array}{l}\text { Objecatives } \\
\text { and Resource } \\
\text { Allocation }\end{array}$ & $\begin{array}{l}\text { Steps and } \\
\text { Their Sequence }\end{array}$ \\
\hline INTERFACES & $\begin{array}{l}\text { Time, People, } \\
\text { Maierialle, } \\
\text { and Other } \\
\text { Resources }\end{array}$ & $\begin{array}{l}\text { Dectsionsh } \\
\text { Procedurea } \\
\text { and } \\
\text { Procesces }\end{array}$ & $\begin{array}{c}\text { Efrort } 10 \\
\text { Do Job }\end{array}$ & $\begin{array}{l}\text { Materials } \\
\text { and } \\
\text { Machinery }\end{array}$ \\
\hline CONSTRAINTS & $\begin{array}{l}\text { Resource } \\
\text { Availability and } \\
\text { Requirements }\end{array}$ & $\begin{array}{l}\text { Steps Needed } \\
\text { to Allocate } \\
\text { Resources }\end{array}$ & $\begin{array}{l}\text { Work Needed } \\
\text { to Carry } \\
\text { Out Sreps }\end{array}$ & $\begin{array}{l}\text { Status of } \\
\text { the Wort }\end{array}$ \\
\hline
\end{tabular}

Figure 5. Operational criteria are matched to different endeavors. 
Accomplishments

In Information Systems Studies, we worked on nine studies during the second grant year. We added two new studies, one long-term and one short-term. The long-term study was PB\& C steering committee. The short-term study was strategic technology processes. Six studies were completed during the second grant year. In Information Systems Studies, we moved the $P B \& C$ work to a contract-funded account in August 1990 and put the long-term overview study on hold. DOE's decision to select WIN as its leading data clearinghouse effectively concluded three of the studies (WIN Interface Study, IDB/SWIMS Interface Study, and the Environmental Restoration and Waste Management Data Base).

In the Long-Term Overview Information Study, we completed the first phase of waste tracking systems and moved to information systems supporting five-year planning. In waste tracking systems, we researched the problems in consensus having the right information at the right time. We began detailed analysis of data fields found on waste characterization forms in the information systems survey. We needed to know what kind of information $E M$ is working with in terms of its information systems, not only at headquarters but throughout the organization in the field. We studied numerous unrelated information systems in EM. We also began identifying what the real information needs were and how to get information to the people who need to reach at consensus. We identified the capabilities of information systems supporting waste tracking activities and compared these capabilities to DOE needs. This comparison identified improvement areas for waste tracking and provided the basis for a conceptual approach for accessing consistent waste data throughout DOE. We used this conceptual approach to identify specific tasks to achieve the overall goal of consistent waste information for consensus. We put the long-term overview information study on hold until further notice from DOE.

The PB\&C Steering Cormittee, a DOE steering committee of high level DOE managers, was formed to decide the future of DOE's culture and management structure. This included planning, budgeting, and controlling systems. The goal of this group was to define the data and information needed by DOE managers to exert oversight and authorization functions for Environmental Restoration and Waste Management. The group established the fundamental goals and structures of an integrated planning, budget, and control system. It made a series of changes to the ADS forms; the Program Summary Documents; and other planning, budget, and control documents. The PB\&C Steering Committee, with MSL support, was asked in August 1990 to establish detailed methods for formulating the FY 1993-1997 Environmental Restoration and Waste Management Five-Year Plan. Because this new task did not directly support the consensus research on this grant, it was moved to another funding source.

In WIN Interface Studies, we contributed data gathered from the ISS Volume I, Waste Tracking Systems, and accompanying material from the data input forms gathered for that survey. Based on this and other data, DOE decider to use WIN as a data clearinghouse for all waste data for 
headquarters. WIN managers will use data collected in this study to support this WIN restructuring. This study has been completed.

In IDB/SWIMS Interface Studies, we contributed data on IDB and SWIMS from the first volume of the ISS survey on waste tracking systeins. From this data, DOE managers concluded that SWIMS was ineffective in capturing data to monitor low-level wastes based on the changing nature of waste reporting requirements and the age of the data system. Rather than try to upgrade the system, DOE managers decided to incorporate SWIMS data into a unified database on WIN in Oak Ridge. This study has been completed.

In Environmental Restoration and Waste Management Data Base (EMDB), we contributed data from the first volume of the ISS survey on waste tracking systems and made design recommendations for national waste tracking databases based on our research. Our recommendations were used by the WIN support and development staff to design this database on WIN. This study has been completed.

In Strategic Technology Processes, we began researching strategic planning and management processes as they relate to strategic technology development processes. As technology is developed to ensure DOE activities don't compromise a safe environment, we'll study the associated management processes. This study is in its early stages.

Presentations and Papers are included in Volume II, Papers, Presentations, and Conferences. 


\section{THIRD-YEAR PERFORMANCE REVIEW}

Consensus research during the third grant year has shifted toward increased activity in the fundamental research domain. As the following paragraphs explain, this shift in emphasis is the result of the historical context of our research on test sites and the consensus process.

More than any other aspect of our research, test sites and process studies have been influenced by history. So, during the second grant year we acknowledged that there were no guarantees the Secretary of Energy would continue his emphasis on consensus groups over the lifetime of the grant. During the latter half of that year, we capitalized on the fortuitous events which made possible our focus on test sites and process studies. Although this emphasis occurred earlier than we had planned when writing the proposal, we were eager to accommodate and benefit from history. Our active participation in research concerning test sites and consensus process ended late in the second grant year. However, our observation and data gathering continued and will continue beyond year three. By this observation and data gathering, we'll be able to incorporate findings from current and future group process meetings into our research planning and results.

The adjustments to the sequencing of our research, indicated above, required us to simultaneously observe groups, gather data, and develop new frameworks and models. This change in planned emphasis necessitated that we reorient the phasing of our research, particularly by expanding the fundamental side of our research in the third grant year. This we have done. Thus, there's a noticeable shift away from process and test sites and information systems toward fundamental research. However, we remained actively engaged in a variety of research projects in the two applied research domains.

During the third year, we continued to emphasize theory and concepts, frameworks and models, and consensus tools for methods and rules. We made progress in our efforts to measure consensus. And we came closer to understanding the role of critical variables on consensus outcomes. These efforts will be described more fully in the following pages.

The emphasis originally planned for year three is shown in Figure 2. From the figure, we have extracted six effort areas and three research domains we' 11 use for reporting research in this annual report. The six effort areas are: theory and concepts, test sites, consensus tools (for methods, rules, and the guide), frameworks and models, consensus methods, and consensus process. Within the six effort areas there are three research domains (fundamental, consensus process, and information systems). Regarding sequencing of our efforts, obviously g] obal confirmatory research (such as that culminating in the guide) will dominate the later years of the research grant. Such an integrative effort will be preceded by numerous specific confirmatory studies. The following domains were the planned foci of our third-year research efforts : 
I. Fundamental Research

$$
\begin{aligned}
& \text { Theory and Concepts (exploratory) } \\
& \text { (paradigm-oriented literature search, case } \\
& \text { studies supporting a consensus paradigm, } \\
& \text { frameworks and models, and more effort on } \\
& \text { consensus gathering expertise) } \\
& \text { Consensus Tools for... } \\
& \text { Methods (specific confirmatory) } \\
& \text { (refine instruments to measure consensus) } \\
& \text { Rules (specific confirmatory) } \\
& \text { (relate specific independent variables } \\
& \text { affecting consensus methods and processes) } \\
& \text { Frameworks and Models (exploratory) } \\
& \text { (paradigm development related to case otudies) }
\end{aligned}
$$

II. Consensus Processes

Test sites (specific confirmatory)

(routinize consensus group set-up)

Consensus Tools for...

Rules (specific confirmatory)

(relate environmental issues to the consensus process)

Consensus Methods (exploratory and specific confirmatory)

(evaluate selected methods in consensus groups)

Consensus Process (specific confirmatory and global confirmatory)

(design and build tools based on results)

\section{Information Systems}

Test sites (specific confirmatory)

(relate information sources to Five-Year Waste

Plan execution)

Consensus Tools for...

Rules (specific confirmatory)

(evaluate information flows for Flve-Year

Waste Plan, consensus groups, and others)

Domain I for the third grant year is dedicated to: 1) continuing the paradigm-oriented literature search, 2) developing instruments to measure consensus and the dependent variables of consensus, 3) operationalizing the independent variables, 4) developing instruments to measure the independent variables, 5) tying the literature to the consensus paradigm to justify the paradigm, 6) networking to apply different tools to targets of cpportunity, 7) conducting formative studies on tools to get consensus, 8) identifying which tools affect other tools, 9) prototyping consensus methods, 10) setting up experimental studies to examine frameworks, tools, and methods, and 11) developing scenarios for waste cleanup and environmental remediation.

Within Domain I during the third grant year, we refined our definitions of consensus-related variables. We brought in more students in a coordinated effort to focus on the tools for methods and rules for consensus. We also improved our measurement strategies. This will 
Ultimately enable us to determine whether consensus has occurred and to what extent it has occurred within the groups we study, In Domaln II and III, we set up the opportunlty to gathar additional data, Research in these domains, comblined with our fundamental research, has prepared us to integrate our findings into a consensus guide.

Domain II for the third grant year is dedicatad to: 1) identifying specific rules for the gaining of consensus, 2) revising and updating the Flve-Year Waste Plan based on research and development, 3)

identifying and understanding rules to such an extent we can teach others, 4) deriving methods and rules for routinaly updating the Five" Year Waste Plan, 5) evaluating how reorganization and environmental. changes relate to culture change, 6) establishing $r$ decision trea to choose tools and methods in given situations, 7) evaluating results of consensus groups, and 8) researching and applying salected consensus methods in different situations.

Within Domain II during the third grant year, we studied the consensus groups we established and developed criterla and alternatives for conducting meetings of and applying tools to the consensus groups. We observed and evaluated consensus groups during the latter part of year three.

Domain III for the third grant year is dedicated to: 1) inftiating a review process for a conceptual approach to assess consistent waste data, 2) identifying what information should be provided to the conveners, facilitators, and particlpants at the test sites, 3) evaluating communication techniques for representing output of consensus groups, 4) evaluating information-gathering techniques for the Five-Year Waste Plan, consensus groups, and others, 5) observing a DOE steering Committee Consensus group, 6) developing criteria for a support gulde for implementing the Flve-Year Waste Plan, 7) developing criterla for field offices to use in putting together their plans and budgets, 8) determining relationships among strategy, planning, organization, and information, 9) developing criteria for a hlerarchical planning process, 10) upgrading and determining additional equipment and resources noeded for the information gathering and communications research environment, and 11) identifying methods for communicating information.

Within Domain III during the third grant year, we evaluated the information people had avallable to them as they carried out the Five. Year Waste Plan both at headquarters and in the fleld. We addressed several questions. What is the right policy or philosophy for how all those information systems work together? Which information systems feed other information systems? Which information systems are similar enough to provide compatible Information to a decision maker? Decision makers are going to be more involved in consensus-type decisions than ever before. We focused on information systems which gave all decision makers more information so they could come together and make more coordinated or joint participative-type decisions. It's a different type of generation of information than DOE has dealt with before. As year three drew to a close, we continued to consider the origins and flows of information into the Fivo-Year Waste Plan as we participated in the update process. 
Fundamental Research

\section{Background}

In the third year of our research, we've made constiderable progress refining our definitional framework, our consensus paradigm (or conceptual mode1) and 1 ts relevant variables, and our measurement strategles, Our paradigm is 11lustrated in Figure 6. We constructed a revised draft of the conceptual model which outlines the "7 P's" involved in consensus: precipitator, purpose, people, problems, participation, process, and products. The preclpitator is the general or overriding problem the group decision making will address. It's the reason eliciting the meeting. Purpose is the desired outcome. The people are the individuals included in the groups or individual characteristics. The problem relates to the task problem. Participation is the desired involvement of group members. We defined process as the actual steps to obtain results. And products are outcomes and outputs of the decision-making group. We looked at variations of components of the model to determine practical applications. We're using this model to structure the remainder of the 11 terature search. Theses, dissertations, and other studies will address components of our revised conceptual model. We also focused on tool-development and tool selection research. We'll elaborate on these accomplishments in the following section.

Consensus has been defined in the 11terature as either a process to reach agreement or the product (end state) of agreement. This dual definition appears to blur the independent and dependent variables. Because of this problem, the state-of-the-art literature sometimes obscures specifications for the effective design of consensus strategles. Because our conceptual model and our research acknowledges this problem, by distingulshing between process-related varlables and outcome variables, the model's likely to provide useful answers to the question of how to lead groups to agreement. The decision-making process must be observed, analyzed, and improved by implementing tested techniques to improve process effectiveness and to maximize consensus outcomes (such as perceptions of consensus, actual consensus, and decision quality). 


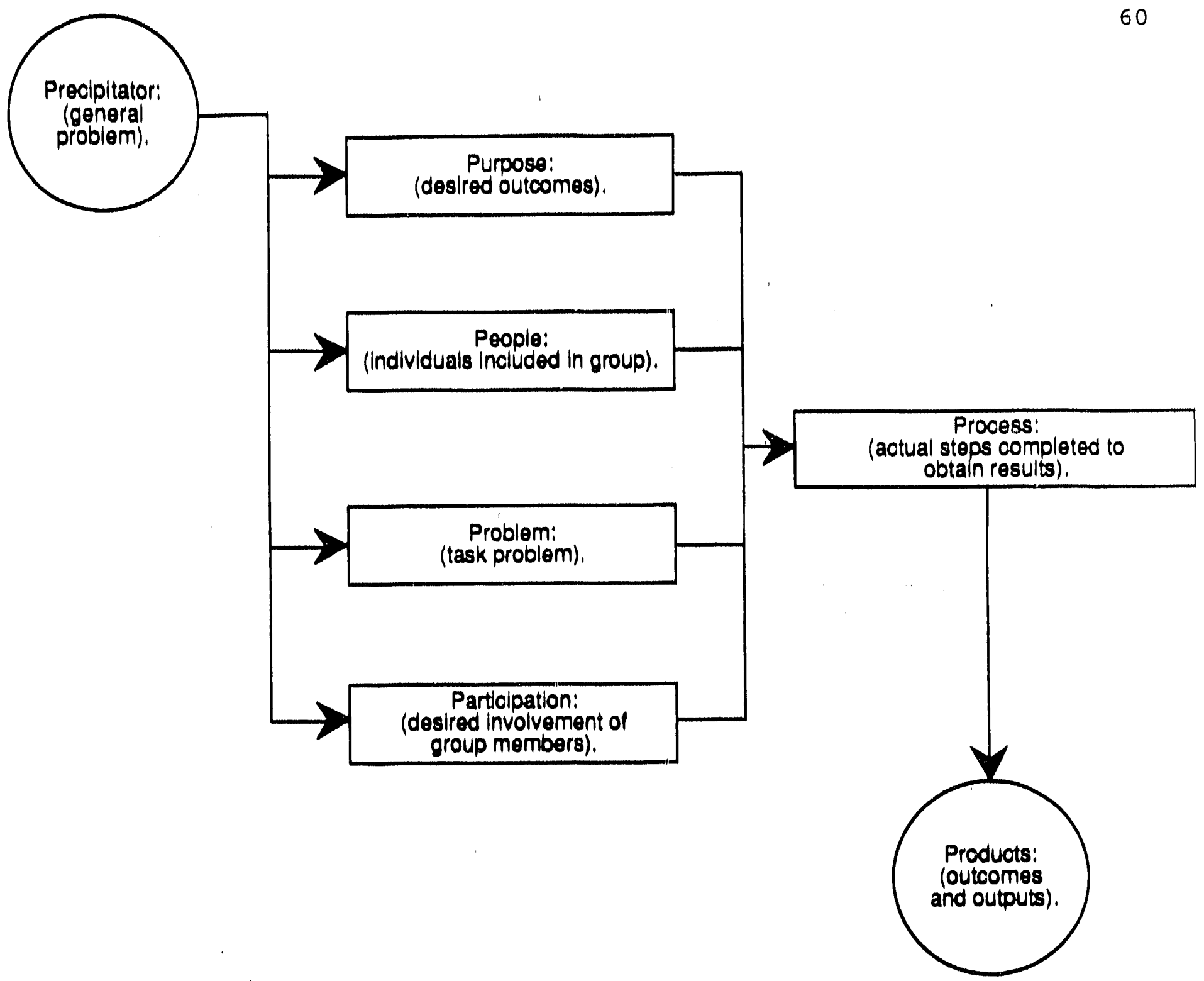

Figure 6. Our paradigm 1llustrates consensus, the variables affecting 1t, and the interralationships of the variables.

We define consensus as a state wherein a common judgment has been achieved by most of those concerned. A fudgment can be a decision or an opinion. Therefore, consensus as an end-state only makes sense when measured at the group level of analysis ( 1 ,e, a consensus, by definition, must be agreement among most members of the group).

However, for perceptual measures of consensus, we can only measure group consensus by aggregating data of individuals within the group. This poses a unit-of-analysis problem that must be recognized.

Two kinds of consensus outcomes are of interest: strength of consensus and quality of decision. Strength of consensus may be further broken down into actual and perceived consensus. Sometimes we can measure actual strength of consensus using methods we have identified from the literature and are refining. One example is a situation wherein we're concerned with the extent of agreenent or convergence of group members in ranking situations. Kendali's coefficient of concordance can be 
applied to data in ranking decisions. The standard deviation can be used to determine "spread" or varlability of the data outside ranking decisions. This measure of spread or dispersion can indicate how "tightly packed" the data are and hence provide an indication of the strength of consensus. We are continuing work on additional ways to measure consensus strength. Measures of consensus strength, both perceptual and otherwise, recelved increased attention as the third grant year moved into its second half.

Decision quality is an indication of the correctness of a decision. In some situations (for example, those which involve ranking decisions) and with some data, (those which involve objectlve data), we can determine accliracy of decision. However, in other decision contexts we cannot know whether the decision is correct or not.

The fact that decision-making groups usually'strive to gain consensus on the highest quality decisions possible plays a central role in our hypothesis formulation. To reach high-quality decisions, we hypothesize groups need to maximize the beneficial effects of shared understanding. We also hypothesize groups require optimal levels of information, expert power, and conflict. These and other hypotheses were the focl of both our field study and laboratory investigations during year three. The results of these studies will enable us to write our gulde to consensus in year five.

Consensus may also be intra-group (agreement within a particular group), Inter-group (agreement between two or more groups), and extra-group (agreement with an agent or individuals outside the group). Our research has addressed each of these types of research, but has pald particular attention to intra-group and extra-group consensus.

From our real-world laboratory (DOE-sponsored meetings of STGWG and DOE), we have compiled considerable data and experience concerning group processes in consensus meetings. This data will enable us to better design group-process research and intervention strategies and will set the groundwork for our guidebook on group process. We'll expand on these findings to date and our plans for this data set in a later paragraph.

\section{Accomplishments}

For fundamental research, we worked on fourteen studies during the third grant year. During the third grant year, we added two new studies: consensus-based planning and environmental trilogy. We completed six. studies: organizational statics and dynamics, consensus gathering systems, consensus scenario generation, information availability, consensus based planning, and consensus case study research. We moved Consensus Process Studies under the applied area.

In Consensus Library, we expanded the capability of MSLSearch by increasing the flexibility for entering the article summaries and by making the program more user friendly. More work on this effort will continue into year four. The primary purpose of MSLSearch will remain the same ( $1, e$, serve as an on-line data base to be used by graduate 
students, MSL and adjunct researchers, and associated research faculty); but the version 1.1 , will be able to store more articles, reduce the amount of time needed to load the information, and enhance the users' ability to retrieve needed information.

We've continued to locate and review articles. In year three we identified 882 articles we want to review and reviewed 223 of these. We also began the process of categorizing our variables. From the literature we've developed 24 broad categories of variables we believe are relevant for studying consensus. In addition, we also improved our earlier paradigm of consensus and have begun to use it to organize our research.

We began to fill the gaps in the lf.terature as our ongoing research has become more precisely defined. For example, we now have ongoing studies in such areas as conflict and consensus, the effect of expert knowledge on consensus, and how different corsensus gaining techniques affect consensus. In addition, we continued our literature-based efforts on how consensus can be measured. Finally, we've fust begun to study consensus gaining and decision making in specific planning groups. Each of these studies requires extensive literature reviews. Thus we've broadened our literature base, but have become more specific in the type of literature we're reviewing.

In Consensus Process Studies, we concluded one research effort concerning intra-group consensus. (Consensus Process Studies includes intra-group, intergroup, and extra-group.) We found that without facilitation, consensus on important issues may not be achieved. As indicated above, we also collected considerable data from meetings of STGWG and DOE (extra-group consensus). We began analyzing and interpreting our data during the closing months of the grant year.

As part of our efforts under this research, we designed the perceived consersus instrument by modifying questions used by Knutsen, Lee, and Danes (Knutsen \& Holdridge, 1975) and adding two questions from an instrument used by DeStephen (1983). The original questions derived from knutsen et al. were derived from several q-sorts. Items kept were those judged most important to distinguishing between agreeing groups and disagreeing groups. Sixteen items were scaled from one (strongly disagree) to seven (strongly agree). Cronbach's alpha for the measure was .92 suggesting good internal consistency. The perceptual measure is assessed at the individual unit of analysis. When determining consensus in a ranking task (i.e. how much group members agree in their rankings), Kendall's coefficient of concordance is an appropriate statistic to use to index group-level agreement.

Additional research was also conducted to characterize two constructs: individuals' perceptions of information usefulness and shared understanding among consensus group members. These constructs are process variables we hypothesized as important to consensus outcomes.

We wanted to determine if the strength of consensus in small groups could be predicted from group members' perceptions of information usefulness and shared understanding. The hypothesis was that group 
members' perceptions of shared understanding (Tfosvold \& Field, 1985) and information usefulness (Larker \& Lessig, 1980) would relate to their ratings of strength of consensus. In other words, subjects perceiving that the information shared in the group was particularly relevant to decision making would perceive strong consensus. Conversely, those perceiving little information usefulness would perceive low consensus. The rationale behind this hypothesis was that the infusion into the discussion of helpful information would facilitate cooperation and agreement among participants. In addition, group members believing that there was considerable shared understanding in the group would perceive strong consensus. Conversely, group members believing there was little shared understanding among group members would believe that consensus was low. Furthermore, it was predicted that the combination of perceived information usefulness and shared understanding would predict strength of consensus better than either predictor alone. Results indicated that perceived information usefulness was not
significant factor. In the prediction of strength of consensus $\left(R^{2}=.099\right.$ $(p-.08)$. On the other hand, shared understanding did significantly predict strength of consensus ( $\left.R^{2}-.67, p<.0001\right)$. These results suggest a substantial portion of the variance of consensus was accounted for by shared understanding and confirms earlier work in this regard. The hypothesis that perceived information usefulness and shared understanding would combine to significantly predict strength of consensus was not confirmed. This research was completed in year three.

An additional research effort related to observing and analyzing consensus meetings. Here, we summarize data collected from meeting evaluation forms at three meetings: the Atlanta and Arlington State and Tribal Government Working Group (STGWG) meetings in April and May 1991, and the Arlington Stakeholders' Forum in May 1991. This form measured participants' perceptions of their opportunity to express views (four questions), the degree their expectations were met (two questions), and their perceptions of DOE (openness, willingness to share information, willingness to act on suggestions; four questions). The form also asked for unstructured comments on the logistics of the meeting. This form served two purposes. First, it gave MSL valuable feedback on how to improve future meetings (STGWG and others). Second, it supports our research on consensus.

For each meeting we determined the response rate for the evaluation form, the means for the responses to each question, and the complete comments written on the unstructured feedback section. We've also summarized comments that were given by more than one person and analyzed the collected data. We've investigated the reliability of the evaluation form's scales using Cronbach's alpha. We've calculated means to check for differences between meetings, sessions, or groups.

Response rates for the three meetings ranged from 478 to 818 . This resulted in sample sizes ranging from 14 to 19 for our statistical tests. These sample sizes were slightly lower for tests run without DOE responses. These low sample sizes limited our ability to run statistical tests, and reduced the statistical power of our tests. 
The multi-item scales we used (perceptions of opportunity to express views and participants' perceptions of DOE) were reliable for all three meetings. (Cronbach's alpha was greater than .80 in all but two cases.)

In all three meetings, participants' perceptions of their opportunity to express views were related to perceptions of met expectations.

In STGWG meetings, the degree facilitator-set expectations were met was related to the degree the participants' personal expectations were met. This result implies either that STGWG expectations were not too dissimilar from the facilitator's expectations or that both sets of expectations were met in the meeting.

There was "consensus" among individual participants on the ranking of the 10 issues as determined by Kendall's coefficient of concordance. However, this consensus was not very strong.

Further research on this project will continue late into the grant year and has been transferred to Consensus Process Studies section in the applied research area.

In Environmental Trilogy research, we began conceptualizing a model encompassing the technical, institutional, and cultural perspectives that converge on environmental issues. The model will be developed further in year four.

In Consensus Paradigm or Conceptus 1 Model Development, we continued to refine definitions to identify relationships among the variables of consensus found in the discipline-oriented 1 iterature search.

Fundamental or basic research paradigms give us the concepts to develop models of EM and ways to measure consensus and other elements of EM. We constructed a revised draft of the conceptual model which outlines the "7 P's" involved in consensus: precipitator, purpose, people, problems, participation, process, and products. The paradigm is described earlier in this report.

Organizational Statics and Dynamics research was closed due to shifting priorities.

In Consensus Academic Plan, we revised and updated our plan for approaching our research activities both within each of the three research domains and across domains. The plan provides assistance for maintaining an integrative understanding of consensus as we study consensus outcomes and consensus processes across the three domains.

In Consensus Case Study Research, we studied and compared the specific historical case studies involving consensus processes we selected in the first grant year. We examined key decisions made by consensus in four areas: foreign policy/peace negotiations, emergency relief, Presidential advisory groups ("kitchen cabinets"), and labor/management negotiations. We identified elements present in the successful decisions, as well as those absent in unsuccessful decisions. Through this process, we identified essential elements of a successful consensus decision. These elements are: 1) a strong facilitator; 2) possession of all the relevant 
information needed to make the decision; and 3) shared understanding among all participants.

The facilitator must be a strong leader, but must not stifle discussion. Shared understanding is essential for group cohesiveness, which in turn reduces dysfunctional group conflict. One caveat is that group cohesion must not be allowed to produce "groupthink." People need all relevant information to make an informed decision. Knowing the essential elements and how to foster them will enable convenors and participants of consensus meetings to conduct more effective meetings. From this historical case study analysis we've begun examining the role of shared understanding and information sufficiency in consensus outcomes reported under Consensus Process Studies. This research has been completed.

Consensus Gathering Systems research was closed due to shifting priorities.

In Consensus Group. Dynamics, we continued exploring the group dynamics literature. As suggested by the literature, we further examined independent variables affecting consensus of a group. For example, in a pilot study we examined the effect of expert power on consensus. We learned that group members with information can make a contribution to group effectiveness, even if their individual decisions would otherwise be poor. Facilitators must assure experts contribute all relevant information. We also found that perceptions of expertise, rather than true expertise, were more important to consensus group members. Also, decision acceptance was not related to whether or not an individual thought others would accept the decision. These exploratory findings were also examined further in subsequent studies.

During the remainder of the third year, we expanded this research to explore the effect of expert presence on the quality of decisions. We investigated whether groups with an expert have higher perceptions of decision quality than groups with no expert.

We tested a series of hypotheses related to the use of expert-present consensus-gaining groups. Hypothesis 1 stated that groups with an expert will have better decision quality scores on a Lost-on-the-Moon exercise than groups without experts. The hypothesis was rejected $t(16,3)=0.66, p-.52$. The likely explanation for the failure of Hypothesis 1 was the ineffective manipulation of the expert condition (the manipulation check was insignificant). Further refinement of the manipulation is indicated to provide an adequate test of the hypothesis.

Hypothesis 2 stated that members of groups with an expert will have higher perceptions of decision quality than members of groups with no expert. The hypothesis was not supported. Results were $t(17,6)-.23$, $p>05$.

Hypothesis 3 stated that the higher the group members' perceptions of opportunity to express views, the stronger the consensus. Correlations were computed between perceptions of opportunity to express views and the three measures of consensus. There was no support for this hypothesis. 
Hypothesis 4 stated that the higher the group member's perceptions of decision quality, the stronger the consensus. Correlations were computed as follows. For perceptions of decision quality and the difference measure of consensus, the correlation was $r=.56, p<.01$. For perception of decision quality and the questionnaire measure, $r=.79$, $\mathrm{p}<.001$. For perception of decision quality and the concordance measure, the results indicated an $r=.43, \mathrm{p}=.06$.

Hypothesis 5 stated that the higher the group members' perceptions of the opportunity to express views, the higher their perceptions of decision quality. The correlation between perceptions of opportunity to express views and perceptions of decision quality was $r=.52, p<.05$. If subjects perceive that their views were heard, they're more likely to believe the decision quality is high. Conversely, subjects who agree with the decision may believe their views were heard anyway.

Hypothesis 6 stated that experts will be perceived as leaders. Of the nine experts receiving the highest rating on the use of expert power, five received the highest scores on leadership emergence. When looking only at experts, the leadership emergence scale and the expert power scale were significantly correlated.

An expanded and refined study was designed to reexamine these results with an improved manipulation. This project was carried out in year four and thus will be reported in the fourth year progress report.

In addition, during year three, we assessed whether three different measures of consensus (difference score), perceptual measure, Kendall's coefficient of concordance (for agreement of ranked responses in a ranking task) measure the same construct. The measures were correlated as follows: The difference measure with the perceptual measure $(r=.56)$ and the questionnaire with the concordance measure $(r=.43)$. It should be noted that, while these correlations are high, there's incomplete overlap. Examining $r^{2}$ values we find the $r^{2}$ 's for the above values .31 and .184 , respectively.

In Consensus Techniques, we continued to study various techniques and, through further research and testing, we'11 improve our techniques as needed. We also developed a matching technique for different applications based on the components of the consensus paradigm. This technique enables facilitators to select the best technique for reaching consensus. We've begun to develop a contour map matching available techniques to problem component examples. This tool categorizes group techniques based on our consensus paradigm. The technique was reviewed as a consensus-gaining technique for use at DOE and elsewhere.

We have also investigated structured meeting techniques and reported our findings in a paper included in Volume II of the Third Annual Progress Report. Specifically, we modified and pilot-tested three structured meeting techniques for $E M$ managers. The techniques included modified nominal group technique (NGT), role playing with another modification of NGT, and tabletop exercises. 
Research is also underway to examine the effects of group members' expectations on consensus outcomes. Tools and methods will then be developed to assist facilitators in identifying and satisfying group member expectations.

In Consensus Facilitators, we continued our study of the role of the group facilitator in the consensus process. We developed a series of recommendations for how facilitators can increase meeting effectiveness and efficiency. We drew parallels between meetings and control theory. Meetings, like systems, have inputs, outputs, and the three system components-controller, plant, and sensor. The facilitator's role is that of sensor and controller. Based on this analogy, we assert that an effective facilitator, or third party assisting consensus, can assist or adapt a group process to accomplish the group's purpose. But group dynamics, rather than the task, is the focus of facilitation. Therefore, facilitators may benefit from knowledge of members prior to the meeting. For example, it's possible some individuals are easier to bring to consensus than others. Future research can examine this hypothesis.

In the third grant year, facilitator studies examined the role of interpersonal conflict in group consensus process. Specifically, we began exploring the effects of different types of conflict on the ability of groups to achieve consensus and/or high-quality decisions. We've hypothesized that optimal levels of conflict may assure that more options are discussed. This study w111 be reported in the Fourth-Yearto-Date Progress Review under accomplishments.

In Consensus Scenario Generation, we began analyzing information to develop DOE-applicable scenarios for problem solving requiring a consensus decision. Using supporting information from the literature search, our qualitative data from observing consensus meetings and our experience testing the Lost on the Moon exercise (under the Organizational Statics and Dynamics study), we had planned to develop a clear definition of the problem and some form of choice for the solution. We'd also planned to choose one scenario and develop it into a management tool for team building. However, this project has been postponed.

In Information Avallability studies, we examined issues such as whether 1) consensus can be reached without all the relevant information and 2) low-quality decisions are due to lack of information. The opportunity for data gathering didn't develop as we would have liked, so this study was closed.

In Consensus-Based Planning, we began a literature review and developed a preliminary framework to guide consensus-based planning processes. We took into account the interaction of group members with differing areas and degrees of expertise. Due to the direction this research has taken, this project was moved to another funding source.

Another consensus-based planning project developed a classification system for managers who are considering using a consensus process during organizational planning. Derived from theory and the literature, the 
classification included four types of planning (autonomous, consultative, committee, and consensus).

Individual or autonomous planning involves one planner having access to all required information and formulating a plan. Autonomous planning is conducted by one planner who may decide to use other people as information sources but who has authority to write the plan.

Team planning may be by a consultation, a committee, or by consensus. During consultative planning, multiple planners provide input. However, final planning authority is not shared with the entire group. One or more planners collect, synthesize, and disseminate a plan following input from the group.

In committee planning, the group divides responsibility, effort and authority, usually by area of interest or expertise. Each subgroup or individual planner constructs part of the plan.

Consensus planning permits planners to reach a common fudgment. Planners share information, express views, and come to agreement about the final plan. (By agreement we mean general agreement, not unanimity.) The following guidelines may assist those deciding on the type of planning to use:

1) Determine whether to use team planning; 2) Determine whether to use consensus planning; 3 ) Determine whether consensus planning justifies the cost; 4) Chose a structured planning process; 5) Choose an interactive participation method for meetings; 6) Choose simple but powerful information and analysis tools.

The benefits of consensus planning are increased acceptance, agreement, willingness to implement, and commitment. Disadvantages include time and effort. However, taking the time to elicit buy-in may save time and effort later. Another potential problem is that consensus planning may result in plans conflicting with the manager's vision. Yet, carefully implemented, consensus-based planning can yield high-quality plans. An expanded version of these findings was presented to a national conference.

Presentations and Papers are included in Volume II, Papers, Presentations, and Conferences.

Consensus Process Studies

\section{Background}

As indicated earlier, the magnitude and impact of the U.S. Department of Energy's (DOE) Environmental Restoration and Waste Program (EM) required a drastic change in DOE's culture. This included the participation of all levels of government, public forum representatives, and the public. This change enabled us to observe consensus processes as groups met and tried to agree on issues. Much of our research focused on capitalizing 
on these opportinities. However, grant year three efforts focused on observation and data gathering rather than a greater level of involvement.

We also observed consensus processes as coordinator and facilitator of Environmental Restoration Program managers' meetings. These meetings were a forum for DOE Environmental Restoration Program Managers at DOE headquarters and its various field offices. Managers exchanged information and shared experiences, worked together on common problems, and discussed specific items on the meeting agenda to elicit thoughts and questions. Through these meetings, we engaged in preliminary work to understand $E M$, identify $E M$ variables, quantify $E M$ objectives, and develop EM paradigms. We'll continue developing real-world instruments and running real-world studies--in the EM environment and in the EM laboratory environment.

At a management coordination meeting in Denver, regular Environmental Restoration Program Managers' Coordination Meetings were held. Observational data collected at these meetings provided a database for our consensus research and generated additional hypotheses.

\section{Accomplishments}

For consensus process, we worked on ten studies during the third grant year. We added two new studies, both short-term, called: the office of Technology Development (EM-50) integrated demonstrations and the office of Environmental Restoration (EM-40) management plan. We completed six studies during the third grant year. Four of the completed studies were moved to another funding source. The four studies were: 1) EM command briefs, 2) continuous information transfer, 3) EM-50 integrated demonstrations, and 4) EM-40 management plan. The other two completed studies were regulatory consensus research and EM primer.

In Management Analysis and Networking, we carefully observed DOE's progress in communicating and promulgating its new culture. We continued to observe DOE headquarters, field sites, contractors, and off-site participants to observe changes.

In Consensus Meetings, 1ittle activity was performed on this consensus study area because of federal budgetary constraints placed upon DOE. However, DOE asked MSL to begin researching issues and planning for an Environmental Restoration Program Managers' Meeting held in May 1991.

One area of consensus group research has been the Stakeholders' Forum. The Stakeholde's' Forum meets once a year to give a broad range of people an opportunity to provide input into DOE's Environmental Restoration and Waste Management Five-Year Plan. The forum consists of representatives from various federal agencies, congressional staff, industry, education, environmental interest groups, and labor unions. A Stakeholders' Forum was held May 19 - 21, 1991 in Arlington, Virginia. Under our grant, we helped DOE to research, design, and facilitate the forum to meet DOE's objective of providing a wide range of stakeholders (those with a vested interest in DOE's environmental programs) an 
opportunity to communicate directly with DOE on issues related to the FY 1993-1997 Flve-Year Plan.

As facilitators, we helped participants to elicit and gather comments on the Five-Year Planning process and related issues, then developed these comments into a report which DOE addressed at the conclusion of the forum. As facilitators and observers we gained valuable data on intergroup consensus bullding.

For the past three years, we've had the opportunity to observa the state and Tribal Government Working Group (STGWG). STGWG is an on-going group which meets approximately four times a year to provide input into the formulation of DOE's Environmental Restoration and Waste Management Five-Year Plan and to raise for discussion with DOE related Five-Year Plan issues. STGWG participants consist of representatives of elected officials from sixteen states, three Indian Nations, and three national government organizations.

Our opportunity to observe this working group has resulted in the gathering of data needed to support our studies of consensus processes. We observed and measured four meetings (May 15-17, 1991 meeting in Arlington, Virginia; and September 29-October 2, 1991 meeting in Denver, Colorado; December 2-5, 1991 in Las Vegas, Nevada; March 17-20, 1992 in Dallas, Texas).

In EM Command Briefs (old Transition Management), we assisted with the restarch and design of fact sheets from information already researched for other study areas. We moved this work under a contract more suitable for sponsoring this type of support effort.

In Continuous Information Transfer (Video), no activity was performed in year three under this grant and because this work became a more service oriented effort, we moved it under a more suitable funding source.

In Regulatory Consensus Research (Land Disposal Restrictions), we worked with EM and EH to complete the research of guidelines for land disposal of waste, and we worked with them to reach consensus on a tool needed to communicate these guidelines effectively to EM, EH, and others. This resulted in the formulation of the Land Disposal Restrictions (LDR) Guide which was published and distributed in February 1991. We worked jointly with EM and EH to develop the LDR Guide.

The purpose of the LDR Gulde is to provide Environmental Restoration Program Managers with information on the application and implication of the LDR regulations to DOE's Environmental Restoration Program. It is intended to assist Environmental Restoration Program Managers plan their projects to ensure compliance with LDR requirements. The guide employs environmental restoration "scenarios" and examples of decision flow charts to guide Environmental Restoration Program Managers through a decision-making process leading to LDR compliance upon project implementation and completion. This study has heen completed.

In the EM Primer Study, we completed the research and design of the EM Booklet, "Environmental Restoration and Waste Management." The booklet 
was designed to provide an overview of the program with minimal text supported by many photographs. The booklet served the needs of those intimately connected with $E M$ and introduced $\mathbb{E M}$ to those unaware of the program. It was publishod and distributed by DOE In Fobruary 1991. This study has been completed.

In Offloe of Technology Development (EK-50) Integrated Demonstrations, we worked with EM-50 on protocol and guidance on integrated demonstrations. We designed and developed definitions and tables of contents for three integrated demonstration guidance documents. Because this new task came to Indirectly support the consensus research on this grant, it was moved to another funding source.

In Offlce of Environmental Restoration (EM-40) Management Plans, MSL was asked to work with DOE and contractor employees to build a prototyps EM Management Plan, using EM-40 as a test program. The process of building this management plan was used not only to achleve consensus on the management plan format and contents but also to achleve consensus on several EM management processes. The group established the fundamental goals and structures of the EM-40 Management Plan, and worked through a series of reviews and changes to obtain consensus. MSL was then asked to make editorial changes and finalize the EM-40 Management. Plan, and transfer the knowledge gained on the EM-40 Management Plan to the development teams of other management plans. This new task did not directily support the consensus research on this grant, and the task was moved to another funding source.

Presentations and Papers are included in Volume II, Papers, Presentations, and Conferences.

In Networking for Consensus (UME), we continued setting up bridges and networks making contacts, having discussions, and interviewing people, providing those people with information, and convincing those people to participate in the new DOE culture. We also provided support to the literature search reported under fundamental research.

Information Systems studies

\section{Background}

Like all managers, DOE managers use information to make dectsions and set policy and strategy. Often, they nust make these decisions based on incomplete information. There are two main reasons for this. First, it's sometimes difficult for DOE managers, with different preferences, to agree on the information's content. When managers have not reached consensus on what's needed, decisions may be based on incomplete information. Second, because of the lengthy concurrence chain, decisions must often be made before information can pass through a timeconsuming approval process. In both cases, improved management of intermediate information through automation can lead to more effective information sharing which leads to more effective decision making. 
Managers at different organizational levels may need different data and information for their decision making. Therefore, managers with differing needs who go to the same formal data stores, may not find what they need. They may be data rich and information poor. If designers of large computer systems don't understand the difference between data and information, they can't meet managers' neads. Designers of information systems must also understand the influence of hierarchloal organizations (the prevalent form) on the design of information systems. These organizations typloally have overlapping domains of responsibility, events or activities performed at the bottom of the organization are directly managed at the bottom of the organization and indirectly managed up through the organizational hierarchy. How does such a structure influence design of information systems?

The deslgn of information systems is still very much an art. We design information systems by looking at the characteristios of information, decisions, and the manager. For information system design to move from an art to a sclence, we need a detalled understanding of data, Information, and the process by which data become information.

No existing comprehensive frameworks defining data and Information sufficientiy describe the data-to-information process. Moreover, there's confusion about the terms "data" and "Information", and the two terms are often used interchangeably according to Appleton (1986). Upon close examination of the 11terature, we found definitions and relationships that each serve to describe a portion of the data-toinformation process. By assembling these definitions and relationships, we've developed a framework to describe data, information, and the relationships between data and information. We're working on defining data and information in enough detall to describe differences in data and information needs at different organizational levels.

For example, Drucker (1966) says a manager converts information to action through decisions. Most managers rely on information tools to provide them data and information. Together, the manager and the tools perform the data-to-information process. The point in the data-toinformation process at which the tools stop and the manager continues the process is the point at which portrayal occurs. Portrayal can be data, quantitative information, or qualitative information. We define four portrayal formats: tables, graphs, checklists, and narrativo. The purpose of portrayal is to convey the results of the data-to-information process to the manager at the appropriate step in this process. The appropriate step should be the point at which the manager is capable of completing the process without further help from the tools.

Portrayal occurs at different steps for different managers and different situations. Managers sometime perform the entire data-to-information process; for example, when a manager walks around his or her domain to observe the operations first-hand. Managers sometime perform 1ittle of the data-to-information process; for example, when a manager hires a consultant to provide qualitative information for decision making. In this case, the consultant is acting as an information tool for the manager. The portrayal interface between manager and tools depends on 
the manager's experlence and knowledge, organizational level, personality type, and the complexity of the problem to be solved.

Information systems should be bulit and operated with an understanding of the data-to-information process they support. The data-toinformation process serves as a gulde to providing the right data and information to managers by showing the data components and data and information types, and how data become information. Further, the portrayal interface betwoen the information tools and the manager describes the right way to provide data and information to the manager. If the tools complete more steps than needed by the manager, the Information tools are inefficlent, and the manager loses the ablilty to use his or her personal preferences in the data-uo-information process. If the information tools complete fewer steps in the process than the manager needs, the portrayal will be incomplete, and the manager will be unable to produce qualitative information in a timely manner. The datato-information process points the way to the effectiveness of the information tools (getting the right data and information to the manager) and the correct portrayal interface points toward the efflclency of the information tools (providing data and information the right way).

For effectlve and efficient transfer of information we must better understand organizational level. Organizational levels are defined as levels of managerial activity or decision making. Managers are fudged to be at the level of managerial activity or decision making they primarlly perform, although all managers may perform some of every activity and decision. Parsons (1960) proposed three levels of decision making in an organization: institutional, managerial, and operational. Institutional decisions are performed primarily at the top of the organization and set broad goals and policy. Managerial decisions are at the middle of the organization and direct the activities of the organization and coordinate tasks. Operational decisions normally take place at the bottom of the organization and involve performing the activities of the organization.

Similar to Parson's framework, Anthony (1965) developed a taxonomy for managerial activity. Managerial activity is broken into three groups: strategic planning, managerial contrul, and operational control. Managers performing strategic planning set policy, organizational objectives, and determine resources tc be applied to attain these objectives. Managers performing management control assure resources are obtained and used wisely in the performance of the organization's objectives. Managers performing operational control are responsible for carrying out specific tasks.

By understanding the target organization and by taking information requirements into account, our information-gathering system will help decision makers obtain the right information (1.e., it will enable users to become information $r i c h$ ) by offering a systematic framework for data gathering. We'll accomplish this by realistically simulating information in the real-world of EM. We'11 plan for year four's integration of computer hardware and software for information gathering and communication. An extension of systematizing information is the 
effective communtoation of that information so consensus groups may make informed decisions. Accordingly, our efforts in the third year also w111 lay the groundwork for the Communirations Research Environment outlined in the proposal. As the continuation proposal for year four suggests, once we've established a realistlc laboratory environment for the gathering of information, we'll be able to experiment with various communications formats.

Our work also included the research and development of consensus tools such as information briefs. EM Information Briefs are a useful tool to communicate the new Office of Environmental Restoration and Waste Management's program activities to a variety of audiences. These audiences included congressional staffers, people new to the EM program, and members of the general public with an interest in the EM program. These briefs were modular in design so they could easily be tallored to meet the intended audience and purpose of the presentation. These briefs were useful in effectively communicating EM's major program activities to a wide range of audiences during a time when EM was growing rapidly.

Besides the design and development of information briefs to effectively communicate EM's major programs, MSL researched, designed, and developed a booklet about EM's major program activities. Once again, the audience for the booklet was congressional staffers, people new to the EM Program, and members of the general. public with an interest in EM's Program. The purpose of the booklet was to be a tool that people could read to learn about EM's major activities. The booklet was used as part of the handouts at congressional hearings, public meetings on DOE policy, and conferences related to DOE's activities. Knowing the audience and purpose of the document, MSL designed the document to contain a minimum of words with a maximum of photos and graphics to describe EM's major activities.

In another study, we were asked to research, design, and develop fact sheets. Many overlapping and conflicting regulations govern the land disposal of waste. The development of a consensus tool was needed to guide EM, the Office of Environment, Safety, and Health (EH) and others in understanding these regulations and their applications. MSL worked with EM and EH to research land disposal guidelines and develop a consensus tool for effectively communicating them.

Some audiences interested in EM's mafor program activities are interested in only one segment of it. For example, if they live near the Savannah River Site (SRS) they might be only concerned with facts related to SRS's program areas and not any of the other operations offices. To meet a particular audience's need, EM asked MSL to research, design, and develop fact sheets ibout EM's major program's at each operations office. There was one fa't sheet per operations office and once again the intended audience was congressional staffers, people new to the EM Program, and nembers of the general public with an interest in EM's Program. 
Accomplishments

In Information systems, we worked on seven studies during the third grant year. We added four new studies: WMIS conceptual design, flveyear plan executive summary analysis, MSLTRAK, and EM hazardous and sanftary waste program background reports. We completed all the new studies except for EM hazardous and santtary waste program background reports. Also completed at the end of year three was strateglo technology processes.

Long Term Overview Information study has been on hold and was closed at the end of year three with no work performed.

In Strateglc Technology Processes, we performed research on a data and information characteristics framework. This research was performed by a masters' degree student at the MIT Sloan School of Management as part of the thesis requirements. It was completed in May, 1991. The research objective was to gain an understanding of the information used in strateglc decision thought processes. With today's increasing importance of strategic decisions there is an urgent need to find new ways to support strategic decision making. Often strategic decisions are made without adequate supporting information. We simply don't know how to identify what information is needed or used for these non-routine, highly qualitative decisions. This research develops a framework model for characteriaing the information usage of strategic decision thought processes. From this understanding, the reasons why some strategic decision support systems (either human, paper, or machine) are useful and why others are not may become known.

The research had three components. First models of nuclear GOA manager strateglc decision thought processes were reviewed. Second, models of information characteristics were reviewed. Lastly, these models were combined to form a data and Information characteristics framework for characterizing the strategic decision information usage of nuclear GOA managers. This framework was then demonstrated using nuclear GOA manager's strategic decision scenarios. This research was based largely on the Elementary Mental Activities Model for Strategic Decision Making (Jones, 1990). For the test cases of this research the decisions and the related information usage of nuclear GOA managers were studied.

The Data and Information Characteristics Framework (DICF) provides a means to understand, define, and deliver the data and information used in a strategic manager's decision process. This framework links cognitive concepts from the Elementary Mental Activities for strategic Decision Making model with the data modeling aspects of the data cube to characterize the data and information use of a strategic manager's decision thought process. The framework (see Figure 7) is comprised of six components:

$\begin{array}{ll}* & \text { Domain of Responsibility } \\ * & \text { Classes of Entities } \\ * & \text { Attributes } \\ * & \text { Time }\end{array}$


* Relationships and Operators, and Values

These six components interact to define a large portion of the manager's data and information needs.

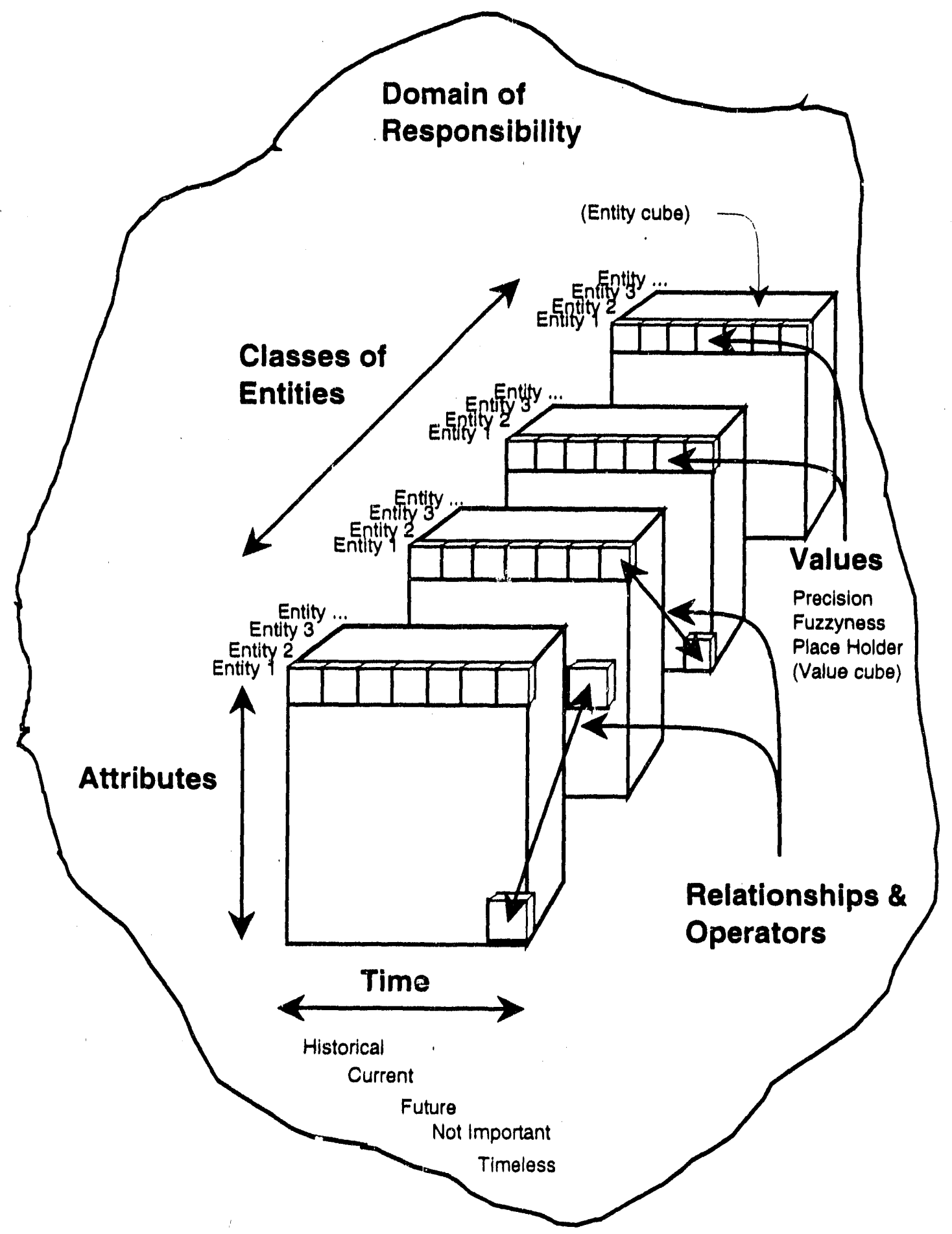

Figure 7. The ciata and information characteristics framework. 
The framework can help establish priorities by looking at the frequency of use of entity-attribute pairs. This frequency of use implies a probability of need for that data and information. Depending on this probability, the information officer can prioritize the gathering and potential development of systems to obtain the d $\mathrm{d}^{\prime} \mathrm{i}$ and information. Work on collecting the information, systems to store it, and building process models to create the information can be ujordinated based on its probability of use.

A unique characteristic of data and information classified as future by the framework's time axes is it determines the need for predictive models to generate that information. Based on its probability of use, determinations can be made of the cost of developing these models and the value of the resulting data and information in strategic problem solving. The same is true for historical time classifications. The DICF model clearly shows what archival systems need to be maintained and their probability of use.

Data and information maintenance is also addressed through the time characteristic. The resources spent maintaining the currency and detail of data and information are significant. From the DICF model the answers to how current is current and how detailed is detailed enough are answered. Based on these assessments and the frequency of use qualifier, one can determine where to apply resources in maintaining currency and collecting detail.

A curious and exceptionally important attribute of the DICF model arises when either the information officer, the manager, or the domain of responsibility changes. This attribute is the inherent training qua-ity of the model.

In the case of a change in information officers, the DICF model becomes a set of easily understood cue cards about how that manager makes decisions and of what information to learn about the organization. The new officer can quickly gain an understanding of what data and information the manager might request as well as know what to get from the organization as a whole.

If there's a change in management, a set of information about "what's important" in the domain of responsibility are readily understandable through the classes of entities and their attributes. In a sense, the manager has access to a structured view of the world according to the available data and information. From this data and information view, managers can apply their knowledge and quickly determine what's missing and what they don't need, if time for administering a new exercise isn't available.

Every decision evolves from some process. This research has begun to define some elementary characteristics of strategic decision processes relating to data and information needs. The result suggests some ways the data and information needs of strategic decision makers might be organized and improved. 
Strategic decision processes are triggered by the identification of a problem and involve the development of an analytic process where data and information are inputs. The result of this process is a decision. The verbal protocols demonstrated the form of these analytic processes and the data and information used in the process. The DICF model provides a means to identify the characteristics of that data and information. This study is closed.

In Five-Year Plan (FYP) Executive Summary Analysis, MSL analyzed the FYP Executive Summary in the summer of 1991 to determine EM's policies and promises to all external groups, as stated in the FYP. EM has management oversight and attendant public responsibilities for the nation's DOE-generated wastes and their environmental effects - - past, present, and future. The EM Five-Year Plan (FYP) is the primary information tool by which EM communicates to the public and regulatory bodies on its progress and plans for managing wastes and restoring the environment. Our goal was to condense these policies and promises and reflect them back to EM so it could (1) evaluate the policies and promises to see if they were correct, and (2) determine whether EM was implementing all the policies and promises. By this method we hoped to promote effective communication between EM and the public and regulatory bodies.

In communications theory, a basic tenet is that, in any communication, the perceived message approximates the intendea message to one degree or snother. If the relationship between what is perceived and what is intended is close, then effective communications can be said to have been achieved. Effective communications is a significant factor influencing consensus. The measurement of effective comrunication and its impact on consensus is part of ESL's communications research. We used the following categories to classify EM policies and promises.

$$
\begin{array}{ll}
\text { * Structure: } & \text { meaning EM's organization and } \\
& \text { methodologies. } \\
\text { * Relations: } & \text { meaning EM's communications channels with } \\
& \text { outside groups. } \\
\text { * Commitments: } & \text { what EM has said it will do. }
\end{array}
$$

We performed qualitative analysis on the Executive Summary and found that EM stressed broad policy commitments, innovative structures, and developmental relationships with outside groups. Of 103 structural references, 70 were related to innovative procedures, such as EM's public outreach programs and technology development efforts. of 92 references to relations, 70 referred to developmental relationships, such as EM's relationships with public groups, environmental groups, and educational establishments. Of 104 commitments, 64 were policy commitments to regulators or the general public. This analys is was submitted to EM in the fall of 1991. No rew activity for this task is planned.

In MSLTRAK, MSL performed the MSLTRAK study in the summer of 1991 to determine the types of tracking and reporting data needed by EM panagers to perform their job. MSL developed a computer system, called MSLTRAK, to be used as a test device for EM managers to evaluate the usefulness 
of different types of data. The source of data used for this test was the FYP Activity Data Sheet narrative, milestones, and budgets from the FY 1993-1997 FYP. The format and content of the data were modified over a period of several months based on feedback from EM managers.

The findings suggest that DOE managers' primary need is to 1 ink budget and milestone data with status data. Status data is data indicating the progress of tasks, both in amount spent and in scope of work completed, against expected completion times and amounts. EM Managers also need to crosscut this information in a variety of ways to determine all tasks meeting a certain criteria, such as all tasks with slipped milestones at a particular location, or pertaining to a certain waste type. Visual presentations were very important, with gantt charts and graphs highly favored to allow quick assessment of data. These findings and others were provided in detail to the Progress Tracking System developers at the study's end in late summer 1991.

In EM Hazardous and Sanitary Waste Program Background Reports, EM's Office of Waste Operations established two new HQ programs this year, the Hazardous Waste Program and the Sanitary Waste Program. EM needed information about hazardous and sanitary waste activities and regulations throughout DOE to determine the scope of these programs, establish program baselines, and develop program objectives and strategies for accomplishing the objectives. MSL was asked to gather this information for EM as part of our consensus research. MSL agreed to review the relevant background materlals and prepare comprehensive reports for the Hazardous and Sanitary Waste Programs so DOE could (1) determine the types of information needed to establish these EM waste programs, and (2) determine the avallability and consistency of waste information throughout the DOE complex. The background reports were to serve as reference material in support of the strategic planning process for program formulation.

The Hazardous Waste Background Report will be produced first due to the availability of hazardous waste information and to better defined needs for the report. It will serve as the model for the Sanitary Waste Background Report. We're currently determining the types of information DOE needs and identifying publications and reports as sources for the needed information. The types of needed information are determined through the report outline. As information is located in field reports, it's placed in the outline of the document as a text section. Data collection for the Hazardous Waste Background Report is expected to be completed in the middle of October, and an initial draft should be complete the first week of November.

In WMIS Conceptual Design, we provided a conceptual design for a Waste Management Information System (WMIS) to support DOE waste management and reporting requirements. One of the major obstacles for waste management in DOE is the lack of a common set of waste data to support consensus decisions. A common set of waste data does not exist because of the various data formats and collection requirements imposed on local DOE sites by state, regional, and other local authorities. To combine these site numbers in various formats into national or complex-wide numbers requires many assumptions and reduces the believability of these 
numbers. Different assumptions can lead to greatly different national or complex-wide numbers, causing EPA-reported numbers to be different than DOE's own internal numbers. Until agreement is reached both within DOE and between DOE and other regulatory agencies and the public about the magnitude and types of wastes that must be managed, consensus about DOE policies and programs will be difficult to achieve.

The WMIS system described in this conceptual design was a central repository for the classes of information required to support consensus decisions for all waste management and operations activities, including waste minimization, treatment, storage, and disposal. Waste management decisions can be broken into two sets: a standard, routine set and a non-routine, imprompeu set. Non-routine decisions can't be anticipated and require special studies or data calls, with much concurrence review and approval, to provide information to support consensus decisions. Standard, routine decisions need data that can be collected and managed in a common database to support consensus decisions. WMIS was designed to fulfill standard, routine information requirements for all DOE Orders, regulations, and EM-30 programs. By focusing on the routine consensus decisions, EM managers can focus on the non-routine decisions requiring different data.

In addition to setting information requirements for WMIS, this document discussed issues affecting the ability of WMIS to support DOE waste management and reporting, and recommended strategies for achieving WMIS objectives.

Along with the report, we presented a briefing on the WMIS conceptual design to field and HQ EM employees. The briefing discussed the conceptual design and answered questions related to the WMIS implementation methodology described in the report. This task was completed in September 1991. No new activity on this task is anticipated.

Presentations and Papers are included in Volume II, Papers, Presentations, and Conferences. 
The emphasis originally planned for year four is 1llustrated in Figure 2. New theorles and concepts, frameworks and models, and consensus process studies were slated to receive very heavy emphasis this year. Also slated for emphasis were tests sites and consensus tools.

As indicated in last's year's progress review, because of the need to capitilize on and benefit from historical events, we emphasized test site, process studies sooner than planned. As a result, we needed to sidulanewsly observe groups, gather data, and develop new frameworks and models. This proverbial "cart-before-the-horse" situation necessitated that we devote relatively more effort to the fundamental side in year three. Again in year four, fundamental. research has received considerable attention. Datails will be specified under the accomplishments section later in this report.

From the figure we have extracted six effort areas and three research domains we'll use for reporting research in this progress review. The six effort areas are theory and concepts, test sites, consensus tools (for methods, rules, and the guide), frameworks and models, consensus methods, and consensus process. Within the six effort areas there are three research domains (fundamental, consensus process, and information systems). We'll discuss accomplishments within the three domains later in this section.

A number of specific fundamental research studies have been completed or are nearing completion. Many of these play an important role in laying the groundwork for more global studies. Regarding sequencing of our efforts, the global confirmatory research (such as that culminating in our structural modeling efforts or our consensus guide) will dominate the later part of year four and year five of the grant. Year four marks a time of increased activity in both the modeling and guidebook activities, as will be described under accomplishments.

On the applied research side, consensus process studies focused on developing conceptual models of the real-world environment. Conceptual models of EM should identify key, programmatic variables and their relationships. For instance, compliance with environmental regulations and operating within the approved budget are two key, programmatic objectives. The conceptual models should (1) identify programmatic variables related to these objectives and (2) relate the variables of budget with the variables of compliance. Anather objective of the conceptual models is to relate the results of the fundamental research on consensus to EM. One result of fundamental research defines consensus as agreement of most of those concerned. For example, consensus about the relative priority of a list of environmental cleanup projects would be measured by having different people or groups of people rank a given set of projects in priority order. One ranking would then be equivalent to one opinion. Consensus would be measured by comparing the rankings of different people or groups of people.

Conceptual models of EM should identify the variables, like the relative priority of environmental cleanup projects, about which we should measure consensus. These conceptual models coupled with the results of 
fundamental research on consensus should enable us to develop

instruments for measurling consensus in the real-world, design real-world experiments, and develop laboratory experiments which are realistic analogues to EM. Specific studies may be organized as follows:

I. Fundamental Research Theory and Concepts (exploratory)

(paradigm-orlented literature search, case studies supporting a consensus paradigm, frameworks and models, and more effort on consensus gathering expertise)

Consensus Tools for... Methods (specific confirmatory)

(refine instruments to measure consensus)

Rules (specific confirmatory) (relate specific independent variables affecting consensus methods and processes) Guide (global confirmatory)

Frameworks and Models (exploratory) (paradigm development related to case studies)

II. Consensus Processes

Test Sites (specific confirmatory)

(routinize consensus group set-up)

Consensus Tools for...

Rules (specific confirmatory)

(relate environmental issues to the consensus process)

Consensus Methods (exploratory and specific confirmatory)

(evaluate selected methods in consensus groups)

Consensus Process (specific confirmatory and global confirmatory)

(design and build tools based on results)

III. Information Systems

Test sites (specific confirmatory)

(relate information sources to Five-Year Waste

Plan execution)

Consensus Tools for...

Rules (specific confirmatory)

(evaluate information flows for Five-Year

Waste Plan, consensus groups, and others)

Domain I for the fourth grant year is dedicated to: 1) conducting formative studies on instruments, 2) collecting data using instruments we've developed during the grant year and prior years, 3) developing frameworks and models accommodating alternate approaches, 4) networking to apply methods to targets of opportunity, 5) conducting integrative studies on guides to facilitate consensus in consensus groups, 6) developing rules for participation, 7) developing a contingency approach for participation (captured in a guide for selecting tool alternatives), 8 ) integrative studies on methods to gain consensus within consensus 
groups, 9) using scenarios in prototype situations, and 10) writing papers and presentations for critical reviews.

Within Domain I during the fourth grant year, we refined our definitions of consensus-related variables. We improved our measurement strategies and accumulated evidence for psychometric properties of consensus measures. We expanded cur 11terature search efforts and began work on the consensus guide. We completed several fundamental research studies related to group dynamics, group process, consensus techniques, and consensus process. We began developing a structural model to test our conceptual model. We developed rules for participation in consensus. oriented decision-making. We also developed a contingency approach to consensus-based planning. We refined our "contour map" for selecting consensus tools.

Domain II for the fourth grant year is dedicated to: 1) developing conceptual models of $E M$ and 2) conducting experiments in the real-world environment of EM.

Within Domain II during the fourth grant year we conducted research in the complex modeling of EM through our configuration studies. We also continued to initiate and facilitate consensus groups in our consensus process studies. This will enable us to continue to observe consensusin-action. Furthermore, we continued to develop and refine measures of consensus. We refined and analyzed a questionnaire for use in consensus groups, as well as worked with a clustering technique that graphically portrays the convergence of individual responses. In addition, we continued to network for consensus.

Domain III for the fourth grant year is dedicated to: 1) improving our working understanding of EM in the areas of information systems and communications systems and 2) developing an EM research environment.

Within Domain III during the fourth grant year, we are bringing computer hardware and software and communications equipment together to establish our laboratory capability. In addition, we have examined the effectiveness of information systems strategies such as the EM Booklet and EM fact sheets.

Fundamental Research

\section{Background}

Interest in consensus is nothing new. In 1921, Park and Burgess (in Scheff, 1984) wrote of consensus. Later, in 1958, Gross urged sociologists to investigate the variable. Meanwhile, efforts to understand consensus have expanded.

The literature on consensus reveals that research on the construct may be divided into two categories: macro-consensus (related to the politics of society) and micro-consensus (related to individuals at the smallgroup level). Within the first category fall sociologists Schelling, Durkheim, and Dewey (Scheff, 1984). Within the second category, small 
group research has proceeded on two fronts: those focusing on process (e.g., Hall, 1971) and those focusing on outcome or the state of consensus (Dess \& Origer, 1987; Gentry, 1984; Rawlins, 1984). The process view considers consensus as the effort toward agreement, while the outcome view considers consensus a state of agreement following group-process activities.

A1though there are many definitions of consensus, a common thread is the notion of agreement. In addition, some definitions include notions of the influence of most group members (Kaprzyk \& Fedrizzi, 1988), the Influence of critical group members (Beck \& Lin, 1983; DeStephen, 1983; Holder, 1972; Price, 1972; Scheff, 1984), and expression of views and acceptance of decision (English \& English, 1958; Tjosvold \& Field, 1985; Wood, 1985).

Some authors define consensus in terms of unanimous agreement (Forsyth, 1983; Hirokawa, 1984; Rawlins, 1984). In their view, if all parties don't agree completely, there is no consensus. Other authors recognize degrees of consensus (Kaprzyk \& Fedrizz1, 1988; Price, 1972). We believe there are degrees of consensus. We call this strength of consensus.

As indicated in last year's progress report, our research efforts defined consensus as a state wherein a common judgement has been achieved by most of those concerned. A judgment can be a decision or an opinion. Consensus as an end-state is measured at the group level of analysis when the interest is whether group members converged. Consensus is measured at the individual level of analysis when individual perceptions of consensus are concerned.

Our fourth-year research yielded a number of results which, taken together, bring us closer to understanding our paradigm or mode 1 illustrated in the Third-Year Progress Report in Figure 6. For example, research reported below looks at the role of purpose, people, participation, process, and products. Of course, any understanding of a construct is a result of careful weaving of the "nomological net" (Cronbach \& Meehl, 1955). This network of theory building relies on the accumulation of numerous investigations by numerous researchers before we can safely assert that we understand the construct. The following accomplishments identify our efforts to make a contribution in that regard.

\section{Accomplishments}

For fundamental research, we're working on nine studies during the fourch grant year. We added one new study called personality variables and consensus and completed three: consensus techniques, consensus facilitators, and environmental trilogy.

In Consensus Library, we continued to expand literature roview and retrieval efforts. The Consensus Literature Review was divided into two stages. In Stage One, the relevant disciplines were defined, key journals were identified, and a list of key words were developed. In Stage Two, relevant articles from the selected disciplines were 
identifled and then reviewed by graduate students who were either working on a thesis related to consensus or were hired to help with the literature review.

Articles come from many sources. Graduate students generally find articles through library research. We've searched the Pscyhlit and InfoTrac computerized data bases for relevant periodical articles. We've searched the Virginia Tech Library System (VTLS) for relevant books. We've also searched the tables of contents of relevant fournals for recent articles thac don't show up in the computerized data bases. The bibllographies from reviewed articles are good sources of other relevant articles.

An electronic search was done in the Soclology, Psychology, Management Science, and Political Sclence literature. Based on the results of this search the following disciplines were added: Communications, Economics, Public Policy, Computer Science, Education, and Engineering/Human Factors. The primary journals in these disciplines were reviewed to identify additional artlules. UME, has searched for articles at the Library of Congress. Thay have sent us abstracts (and some complete articles). We copy, process, and review the complete articles from abstracts we consider relevant.

All people involved fudge the relevance of articles. Graduate students make a relevance judgement when they decide which articles to review. Research associates and graduate students who are more famlliar with the grant make relevance fudgments on what articles to acquire and review. Several factors determine the relevance of an article:

Topic: Articles on topics central to the grant topic are more relevant than topics tangential to the grant topic.

Journal Qual1ty: Articles from refereed fournals are more relevant than articles from non-refereed journals. Articles from academic journals tend to be more relevant than articles from trade journals.

Age: In general, newer articles are more relevant than older articles. The assumption here is that newer articles incorporate the related research up to that date and may make modifications of older work. The exceptions to this rule are seminal articles, such as Janis' work on groupthink.

Author: Articles from authors who are experts in the field are more relevant than articles from other authors.

Graduate students are asked to review articles as part of their graduate research assistantship work. Students pick articles to review from the pool of articles generated by other graduate students, research associaces, and UME. At the start of their graduate studies, students research a wide variety of topics. This allows students to find a topic that interests them. Once students find a topic that interests them, they focus their reviewing on that topic. This topic will evolve into their thesis. One result of this process is that we have covered thesis 
topics in greater depth than other topics. The principal investigator and research associates attempt to assure coverage of relevant areas by guiding and focusing graduate students in relevant directions. The principal investigator and research associates also add cltations to the ilterature review database.

The goal of an article review is to summarize the information contalned in an article. Reviewing articles helps us critically evaluate articles, draw conclusions from articles, and compare articles on the same topic. The article review also makes it easier for people who haven't read the complete article to get the information from that article. The article reviews have been put in a computer database for easier access. Below is a brief description of the major sections of an article review. Not all sections are relevant for all articles.

Citation: The citation section has all the information necessary for referencing the article including author, title, and publisher.

Classification: The classification section includes the topic of the article, key words, and the variables investigated.

Definitions: The definitions section gives all words defined by the author of the article. Writing down the definitions helps us define our terms and compare different author's concepts.

Hypotheses: The hypotheses are the premises that are tested in the article. Hypotheses are useful for two reasons. First, supported hypotheses show relationships between variables. Second, hypotheses help us decide relationships we should investigate in our research.

Synopsis: The synopsis section summarizes the key points presented in the article. The synopsis describes the theory and/or experiment presented in the article.

Results: The results section shows which hypotheses were supported and which were not.

Conclusions: The conclusion section describes what the results mean for researchers and practitioners.

Comments: The comments section gives the reviewer a chance to critically evaluate the article, reference other related articles, compare the article to our research, and describe useful instruments referenced in the article.

Interested parties can access reviewed articles using two computer programs. The first program is a PC-based, File Express data base. This program lets people search for articles by various fields including author, title, topic, and key word. Once articles are identified, the article and the review can be pulled from a file. The second program is a more powerful, mainframe version of the PC-based program. The mainframe version has all of the features of the PC-based program plus 
on-screen access to some of the text from the article review. Many of our reviewed titles are already accessible through the mainframe system. Recent debugging of some software problems has resulted in a more useful mainframe application than we had previously. We will continue to check the malnframe system agalnst the po-based system to assure completeness, From the list of possible titles from our varlous searches identified on the previous pages:

- We have 475 titles entered on our computerized system.

- Of the 475 entered titles, 68 are unrevlewed.

- 35 abstracts awalt sorting, cataloguing, and reviewing.

- 80 additional titles are to be xeroxed, abstracted, sorted, catalogued, and reviewed.

We have not tallied the articles recelving preliminary review, but Judged not relevant. However, it should be noted that relevance decisions for any particular article are made by more than one staff person to reduce the possible effects of blas. We'll continue adding to our 11 terature review database until the end of the grant.

Two volumes will summarize our review of the body of knowledge on consensus. The first, the literature review document, will summarize our review of the scholarly literature relevant to consensus in group decision making.

The second volume, the consensus guidebook, will be based on our literature review and our consensus paradigm or conceptual model. However the guide is a practical handbook for managers who work with decision-making groups. While the focus of the guidebook will be consensus, it will cover other aspects of group decision making relevant to consensus groups. We'll develop the recommendations found in the guidebook by reviewing three sources: 1) the group decision-making ifterature, 2) research on consensus groups done at MSL, and 3) lessons learned from practical experience with consensus groups. The guidebook will answer such practical questions as:

What types of people should I ask to participate in my group?

How should I instruct my group to interact?

What's the right problem-solving technique for my group?

How can I reduce (or increase) conflict in my group?

How can I encourage an esprit de corps in my group?

How do different tasks affect the productivity of my group?

What changes can I expect in my group over time?

Work on both documents is underway and will continue during this grant year. These documents will be complete by the end of the grant.

In Environmental Trilogy research, we developed a model for approaching the environment. The model describes three perspectives for focusing 
our environmental concerns: 1) technical, 2) institutional, and 3) cultural. The technical perspective concerns solentific prinolples, laws of nature, and methods for implementing knowledge of those principles in both preventive and remedial approaches. The institutional perspective relates to regulations, laws of soclety, and policies. The cultural perspective brings human concerns to bear on the environment such as values, norms, traditions, beliefs, behaviors, and attitudes.

Based on this trilogy concept, we inftiated an effort to develop and refine a holistic approach to managing the environment. The approach begins by focusing on the holistic approach, discussing the approach, and defining and modeling 1 ts components. Model discussion began with a small group meeting discussing these issues in Blacksburg. Since then, participants and others have studied issues, models, and perspectives arising from the october meeting to prepare for an expanded meeting of representatives of the environmental community. During the fourth grant year, this activity was moved to another funding source.

In Consensus Paradigm studies, the paradigm, 1llustrated in Figure 6 , portrays the elements we belleve underlie an understanding of the consensus construct. It will be recalled that the model incorporates a precipitator (the general problem eliciting the problem-solving effort), purpose (desired outcomes), people (individuals included in the group), problem (task), participation (level of involvement of group members), piocess (actual steps completed to obtain results), and products (outcomes and outputs).

We have used this conceptual model or framework to design studies reported in this section and have continued discussions concerning necessary modifications of the model. At this writing, the paredigm is a useful and potentially valid representation for understanding consensus. Validity, of course, is not an all or none property, but rather a painstaking accumulation of evidence concerning the legitimacy of the construct and its hypothesized relationships. The paradigm will serve as the conceptual model framing our 1iterature review and guide to consensus.

In Consensus Academic Plan, we have revised and expanded our plan for research examining the relationships between variables in our consensus paradigm. We designed studies, targeting on-time completion by the last grant year of student empirical research, staff empirical research, and the consensus literature review and guide.

In Consensus Group Dynamics, the first pilot study and full study of the role of experts was completed and reported for the third grant year. An expanded follow-up study was conducted in the fourth grant year.

This research examined the effects of an expert on the smal1-group consensus process. This fourth-year research differed from the previous work in this study area in several ways. First, we looked at two types of experts, subject and task. Second, we trained experts rather than try to select people with natural expertise. Third, we increased the sample size significantly from 21 to 77 groups. Fourth we added 
questions measuring information sharing for an exploratory analysis. Finally, we discarded questions measuring the use of expert power and leadership emergence.

The dependent varlables for this study were group declsion quality, opportunity to express views, and strength of consensus. We define decision quality as the closeness of the group's ranking to the ranking developed by NASA experts. "Closeness" was operationally defined as the sum of the absolute differences between the group ranking and the correct ranking determined by NASA experts. Higher correlations indfcate higher quality.

We operationally defined opportunity to express views as group members' perceptions that they had a chance to express their views, and that these vlews were listened to, understood, and considered by other group members. Opportunity to express views was measured using a post-task questionnaire. We operationally defined strength of consensus using two methods. The first method was a strength-of-consensus post-task questionnaire addressing acceptance of, agreement with, and commitment to the group decision by group members. The questionnatre measures two aspects of consensus: Individual agreement, acceptance, and commitment; and perceptions of the group's agreement, acceptance and commitment. We refer to the first aspect as Individual consensus and the second aspect as perceptions of group consensus. We refer to this measure of consensus as the questionnalre measure. For all questionnaire measures, we averaged individual ratings to get group measures. The second method of assessing strength of consensus is a statistical measure of the closeness of individual rankings (Kendall's coefficient of concordance). We refer to this measure of consensus as the concordance measure. This measure was used to investigate the validity of the questionnaire measure.

The independent variable for this study was the presence of an expert and his or her type of expertise. We looked at two types of experts, subject and task and compared them to a control group.

The following paragraph describes the specific hypotheses we teated. The first three hypotheses explore the relationships between dependent varlables. Hypothesis 4 is a broad hypothesis allowing us to explore the effects of an expert on the dependent variables.

H1: The higher group members' perceptions of the opportunity to express their views, the stronger the individual consensus.

H2: The higher group members' perceptions of the opportunity to express their views, the stronger the perceptions of group consensus.

H3: The higher the individual consensus, the higher the perceptions of group consensus.

44: The presence of an expert and his or her type of expertise affects decision quality, perceptions of opportunity to express views, individual consensus, and perceptions of group consensus. 
The experts were drawn from an introductory sociology course. Nonexperts were selected from an introductory psychology course. All groups thus consist of one sociology student (with subject training, task training, or no training) and three psychology students. This pool of people had no special knowledge about the moon or experience with survival exercises.

Expert training occurred approximately one week before the experimental session. Experts in all three conditions were instructed not to reveal they were from a sociology class and had participated in an instruction session before the experiment.

We ran 77 groups of four people for a total of 308 individual subjects. 77 were sociology students; 231 were psychology students. Due to attrition of experts between the training and the experimental session, we ended up with 24 subject-expert groups, 27 task-expert groups, and 26 control groups.

We divided seventy-seven groups into three conditions based on the training of an expert placed into a group of four people. Experts were randomly assigned to a condition for training. Within condition, experts were randomly assigned to groups. Non-expert subjects were also randomly assigned to groups. The three conditions are described next.

Condition 1: Subject Expert: In this condition, experts were trained in knowledge on the moon and physics. Subjects were given a pre-test, a lecture, and a post-test. The test covered moon and physics knowledge relevant to the Lost on the Moon exercise.

Condition 2: Task Expert: In this condition, experts were trained in survival exercises. Experts solved two survival exercises (the Desert and Arctic survival exercises) individually and in groups. The trainer discussed with the experts the correct rankings and the rationale for each ranking.

Condition 3: Control: In this condition, experts were given no training.

We measured decision quality by comparing group rankings on the Lost on the Moon exercise to the expert ranking created by NASA. Decision quality was measured using the sum of the absolute differences between the group ranking and the NASA ranking. We measured consensus, perceptions of decision quality, perceptions of opportunity to express views, and information sharing using a 30-item questionnaire. The information sharing questions were asked for exploratory purposes.

We random $1 *$ assigned experts to one of the three conditions. We taught subject experts knowledge of the moon and physics. Figure 1 is a flowchart outlining the procedure for training subject experts. We taught task experts how to solve survival exercises by having them solve similar survival exercises individually and in groups. Figure 2 is a flowchart outlining the procedure for training task experts. We didn't train experts in the control condition. 
To analyze the data, we used descriptive and inferential statistics. We calculated a decision quality score for each group by computing the sum of the absolute differences between the group's ranking of each item and NASA's ranking. We used two measures of consensus in this study. The first measure (the questionnaire measure) was a questionnaire addressing acceptance of, agreement with, and commitment to the group's decision. The questionnaire measured both individual consensus and individual perceptions of the group's consensus. The second measure of consensus (the concordance measure) was Kendall's coefficient of concordance computed for the five second (post-discussion) rankings for each group. This statistic is a measure of the closeness of the final individual ranks.

To test our hypotheses, we used a group level of analysis. While this reduced the statistical power of our tests, we believe it gave a more accurate description of what happens in consensus groups. We were interested in group consensus and group decision quality, not individual agreement and individual decision quality. Our sample size was the number of groups $(n-77)$. We tested $H 1, H 2$, and $H 3$ by computing Pearson's product-moment correlation coefficients between the dependent variables of the study. We tested $\mathrm{H} 4$ by performing a Multivariate Analysis of Variance (MANOVA) with one, between-subjects, three-level independent variable (presence and type of expert) and four dependent variables (decision quality, perceptions of the opportunity to express views, individual consensus, and perceptions of group consensus). Because this test showed a significant effect on the dependent variables, we performed one-way Analysis of Variance (ANOVAs) to determine the source of the significant MANOVA effects.

This section summarizes the results of our data analysis. The first part shows the results of a reliability analysis on the six scales of the questionnaire. The second part shows the results of my hypotheses testing.

We assessed the reliability of the three scales we used on the questionnaire. We used the individual level of analysis to assess reliability because individuals responded to the questionnaire. The sample size for this analysis was 308 . The perceptions of opportunity to express views scale had six items. The individual consensus scale had eight 1tems. The perceptions of group consensus scale had four items. The information sharing scale had twelve items. Cronbach's alphas are reported below. Reliability for the first three scales was acceptable. The reliability for the information sharing scale was low.

\begin{tabular}{ll} 
Scale & alpha \\
\hline Individual Consensus & .88 \\
Group Consensus & .73 \\
Expression of Views & .85 \\
Information Sharing & .52
\end{tabular}

This section describes the results of our hypotheses testing. For each hypothesis, we repeat the hypothesis and the associated statistical test. We then describe the results of the test. Our sample size for 
the tests was 77 (27 subject expert groups, 26 task expert groups, and 24 control groups) because we used group measures.

Hypothesis 1 stated that the higher group members' perceptions of the opportunity to express their views, the stronger the individual consensus. The Pearson product-moment correlation supported this hypothesis $(r-.68, p<.001)$.

Hypothesis 2 stated that the higher group members' perceptions of the opportunity to express their views, the stronger the perceptions of group consensus. The Pearson product-moment correlation supported the hypothesis $(r-.72, p<.001)$.

Hypothesis 3 stated that the higher the individual consensus, the higher the perceptions of group consensus. The Pearson product-moment correlation supported the hypothesis $(r-.80, p<.001)$.

Hypothesis 4 stated that the presence of an expert and his or her type of expertise affects decision quality, perceptions of opportunity to express views, individual consensus, and perceptions of group consensus. A MANOVA was conducted to uncover differences in three conditions on individual consensus, perceptions of group consensus, perceptions of opportunity to express views, and group decision quality. Results revealed significant MANOVA effects.

One-way ANOVA's were conducted to determine the location of the MANOVA effects. No significant effect was revealed for individual consensus, perceptions of group consensus, perceptions of opportunity to express views $(p>.05)$. ANOVA results were significant for the expertise manipulation on group decision quality $(F-7.54, p<.01)$.

Subject-expert groups had the best decision quality. There were no significant differences between task expert-groups and control group.

$\begin{array}{lll}\text { Cond N } & \text { Mean } & \frac{\text { SD }}{23.56 .34} \\ \text { Subject } & 27 & 10.21 \\ \text { Task 26 } & 31.0 & 31.17 .41 \\ \text { Control } & 24 & \end{array}$

This concludes the results to date from the experts study. We are continuing our analysis of this study and will report additional findings when we update year four.

Also begun during the fourth grant year, but incomplete at report time, is a structural equations modeling of the effects of the presence of two types of experts and personality variables on consensus outcomes. This work is illustrated in Figure 8 .

The figure portrays our a priori representation of the structural model. As the figure illustrates, we'll examine whether group characteristics (such as the presence of a task or subject expert) affects information sharing, opportunity to express views, individual consensus, group consensus, and decision quality. The group characteristic variables and individua?. difference variables are exogenous variables, while 
information sharing, opportunity to express views, individual consensus, group consensus, and decision quality are endogenous variables. We hypothesize that the exogenous variables affect all or some of the endogenous variables. We hypothesize that endogenous variables are affected by at least one other variable. Each arrow is an hypothesized effect of one variable on another. An estimate of each effect will be computed. The particular model is called a block recursive model. It's a block model because no hypothesis is proposed regarding a directional arrow between Individual Consensus (IC) and Group Consensus (GC). It's recursive because hypothesized effects are unidirectional. In other words, the relationship between these variables is, at present, hypothesized to be correlational. The model reflects the ideal in that all variables are seen as leading to decision quality. That is, our major interest in consensus, expression of views, and information sharing is our hypothesis that these variables affect decision quality. The analysis will provide us with direct, indirect, and total effects of each variable relative to decision quality and to each other. Using a Chi-square test for goodness of fit, we will test the fit of the data to the model. The coefficient of determination, $\mathrm{R}^{2}$, will measure the variance in decision quality explained by the other variables in the model. 


\section{The Model}

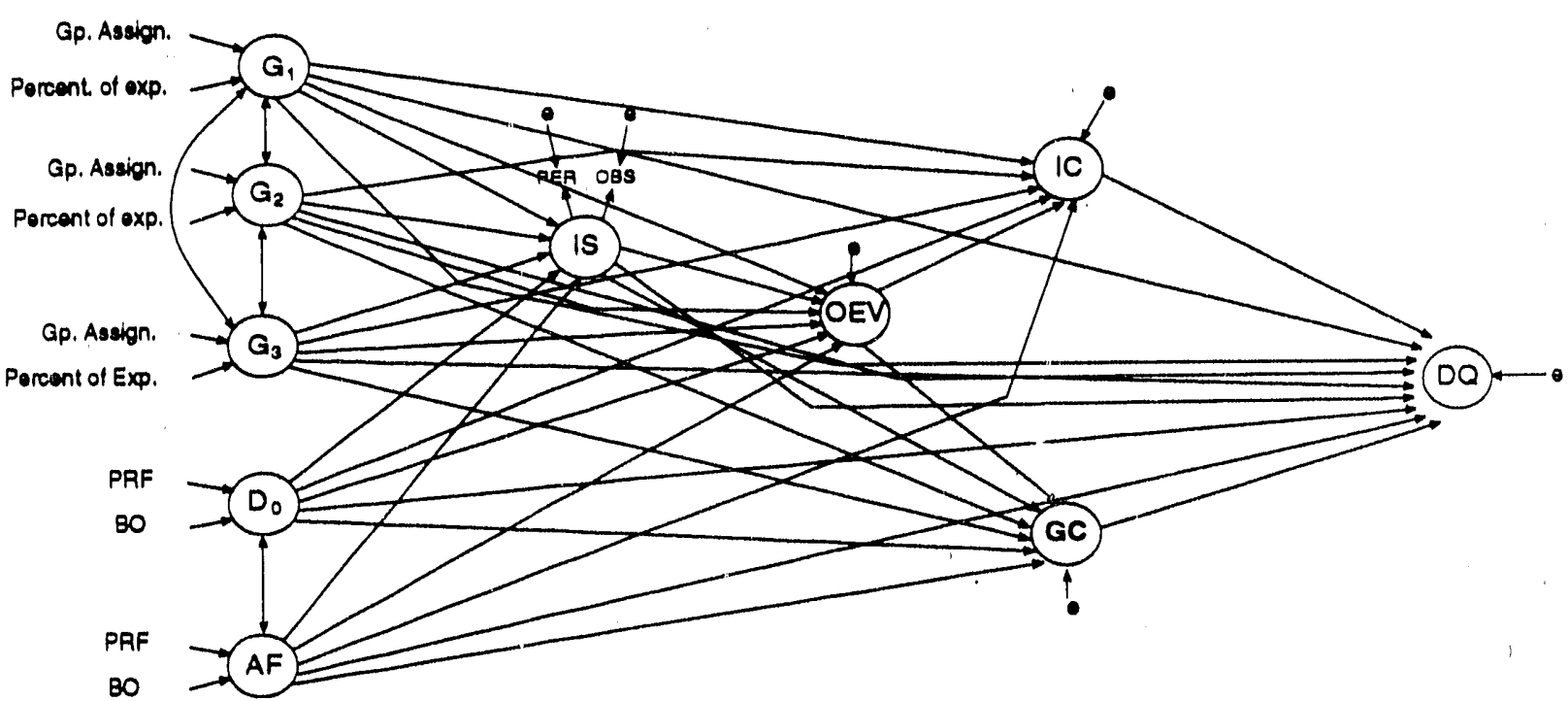

\begin{tabular}{|c|c|c|c|}
\hline VAR & Dofinition & Measure & Role \\
\hline Q1 $_{1}$ & Non-Expert Gp. & $\begin{array}{l}\text { O- not in group } \\
1 \text { - in group }\end{array}$ & Exogenous \\
\hline G2 & Task Expert Gp. & $\begin{array}{l}0 \text { - not in group } \\
1 \text { - in group }\end{array}$ & Exogenous \\
\hline G3 & Subject Expert Gp. & $\begin{array}{l}\text { O not in group } \\
1 \text { - in group }\end{array}$ & Exogenous \\
\hline DO & Dominance & $\begin{array}{l}\text { Scale measure } \\
\text { from PRF }\end{array}$ & Exogenous \\
\hline Af & Affiliation & $\begin{array}{l}\text { Scale messure } \\
\text { from PRF }\end{array}$ & Exogenous \\
\hline IS & $\begin{array}{l}\text { Information } \\
\text { Sharing }\end{array}$ & Concept & Endogenous \\
\hline Por & $\begin{array}{l}\text { Porception of } \\
\text { is }\end{array}$ & $\begin{array}{l}\text { Scalo moasure } \\
\text { (Dalo) }\end{array}$ & Indicator \\
\hline Obs & $\begin{array}{l}\text { Count of information } \\
\text { sharing }\end{array}$ & Count data & Indicator \\
\hline OEV & $\begin{array}{l}\text { Opportunity to } \\
\text { Exprese Views }\end{array}$ & Scale & Endogenous \\
\hline IC & $\begin{array}{l}\text { Individual } \\
\text { Consensus }\end{array}$ & Scalo & Endogenous \\
\hline GC & $\begin{array}{l}\text { Perception of } \\
\text { group consensus }\end{array}$ & Scale & Endogenous \\
\hline DQ & Decision Quality & Kendali's $\tau$ & Endogenous \\
\hline
\end{tabular}

Figure 8. This structural model portrays the hypothesized relationship between type of expertise, personality dispositions of dominance and affiliation, and consensus variables. 
This work will continue into the remainder of the grant year and the results will be reported in our final annual report.

In Consensus Techniques, our efforts at this juncture involve two directions. The first direction is developing a contour map to help managers select consensus techniques. The second direction relates to establishing strategies people can use to enhance consensus.

Last year, we reported that we're developing a contour map to help managers select appropriate consensus-related techniques to use in their groups. We are continuing our woik on the contour map and will complete work on that tool late in the fourth grant year. We'll describe this effort in detail when we update year four in next year's reporting.

Other work has focused on strategies managers can use to foster consensus. Specifically, we've focused this line of research on how to use expectancies within a group process context. We'll describe this research next.

Expectancies are subjective beliefs about the probability of some future occurrence or outcome. Research has shown that expectancies predict motivation (Vroom, 1964), behaviors (Prothero and Beach, 1984; Crawford, Thomas \& Fink, 1980; Eden, 1984; Eden, 1988; Eden \& Ravid, 1982; Rosenthal \& Jacobson, 1968). Rosenthal (1966) describes the effect of what's called self-fulfilling prophesy: "One prophesizes an event and the expectation of the event then changes the behavior of the prophet in such a way as to make the prophesied event more likely" ( $p .129$ ). The Pygmalion effect occurs when an individual's expectations for another become reality. In a complex set of interactions a person may convey expectations to the doer and shape the doer's responses. The expectation thus becomes more likely to happen. The Galatea effect occurs when an individual's changing expectancies result in one's own changed behavior. These two effects are important because they illustrate the importance of recognizing cognitive processes and developing techniques to deal with them. Researchers have also demonstrated that meeting expectations about work environment factors (Porter \& Steers, 1973; Ross \& Zander, 1957; Telly, French, \& Scott, 1971) and job content factors (Lyons, 1971; Ross \& Zander, 1957, Porter \& Steers, 1973) leads to changes in job satisfaction and turnover. Work on procedural versus distributive justice (Folger \& Knovsky, 1989; Lind \& Tyler, i988; Tyler, Rasinski \& McGraw, 1985) illustrate some important points related to such expectations. The first point is that people have expectations about the outcomes they receive and these expectations can affect perceptions of the work context. The second point is that the process (or procedures) by which distribution-related decisions are made may be vitally important to meeting expectations.

By analogy, one might suggest that people have product and process expect tions about consensus meetings. Accordingly, meeting expectations regarding consensus process (1.e., expectations for having the opportunity to express one's opinions or expectations about the degree of conflict within a group) may affect individuals' perception of consensus. Similarly, meeting expectations about consinsus outcomes 
(e.g., decision quality) may influence individuals' perceptions of decision quality and other consensus outcomes.

If these suppositions are correct, then techniques that enable people to meet expectations in the work place ought to have positive effects in consensus-gaining groups.

The following hypotheses were examined:

1) Met expression of views and decision-quality expectations, along with their associated valences, will be a significant predictor of strength of consensus.

2) The higher the degree of met expectations about expressions of views, the stronger the consensus.

3) The higher the degree of met expectations about decision quality, the stronger the consensus.

4) Meeting decision-quality expectations will have a stronger effect on strength of consensus than meeting expression-ofviews - expectacions.

The original model regressed strength of consensus on the following variables:

- Met expectations about expression of views

- Met expectations about decision quality

- Valence associated with expression of views expectations

- Valence associated with decision quality expectations

- Expression of views product sum

- Decision quality product sum

Preliminary data analyses indicated multi-collinearity among the predictors. In other words, high correlations were observed among the predictor variables. This is undesirable. A ridge regression analysis indicated no single predictor variable was significant. To overcome the multi-collinearity problem, a new model was developed. Two predictor variables were used: 1) the expression-of-views product sum, which is the sum of the products of the expression-of-views expectations times their respective valences, and 2 ) the decision quality product sum, which is the sum of the individual products of the dicision quality expectations multiplied by their respective valences.

Strength of consensus was regressed on the expression-of-views product sum and decision quality product sum. The variance of consensus explained by the new model was .136 (adjusted-.117). The standardized estimate or Beta weights for the expression of views product sum and decision quality product sum were .215 $(p-.05)$ and $.215(p-.05)$.

Hypothesis 1 predicted that expression of views and decision quality expectations and theix associated valences would significantly predict individual and group consensus. Hypothesis 1 was confirmed for individual consensus. $R^{2}$ was .101 for individual consensus $(p-.007)$ and 
$.114(p-.003)$ for group consensus. Hypothesis 2 was supported when group consensus was the dependent variable $(p-.04)$. Hypothesis 3 was rejected. Hypothesis 4 could not be tested because neither of the predictor variables significantly predicted the dependent measures. If the results can be replicated, they will have several implications for researchers and managers. For researchers, the results suggest it may not be possible to separate expression-of-views expectations and decision quality expectations from their associated valences. For managers, meeting group member expectations may have positive effects on consensus outcomes.

Some additional exploratory work was conducted under this study classification. Analyses assessed the relationship between the number of verbalizations each group member made and consensus, met expressions of-views expectations, and met decision quality expectations. No significant correlations were revealed.

In a separate consensus techniques project, we researched and developed guidelines for consultative and participative groups. Based on situational leadership theories and following Vroom \& Yetton (1973), the guidelines explain the situations when group-decision making is appropriate and how managers can increase participation should that be desirable. Group decision making should be used when the following situational variables exist:

- The problem is ambiguous (Wynn \& Guditus, 1984; Vroom \& Yetton, 1973 ; Yuk1, 1989;)

- The problem requires generation of multiple alternatives (Bradford, 1984);

- The problem isn't covered by any current policies (Wynn \& Guditus, 1984);

- The benefits are worth the group's time (Wynn \& Guditus, 1984);

- The decision doesn't require any severe time pressure (Vroom \& Yetton, 1973; Wynn \& Guditus, 1984);

- The manager wants to offer subordinates professional development and practice at divergent thinking (Wynn \& Guditus, 1984);

- The manager does not have the necessary expertise or information Bradford, 1984; Vroom \& Yetton, 1973; Yuk1, 1989);

- Subordinates have valuable information or expertise (Wynn \& Guditus, 1984);

- Subordinates are affected by the decision (Vroom \& Yetton, 1973; Wynn \& Guditus, 1984).

Once the manager decides to use a group approach to decision making, she or he must decide whether to use a consultative or participative approach. That decision rests on a number of situational factors. A manager should use a consultative group approach when:

- The decision quality is important, yet the benefits are not worth the extra time for a participative group (Bradford, 1984);

- Subordinates do not have the same goals as the organization (Bradford, 1984); 
- The manager is not willing to accept the risk of sharing with the group the responsibility for making the decision (Wynn \& Guditus, 1984); and

- The manager needs to remain in control (Wynn \& Guditus, 1984).

On the other hand, a participative group approach is appropriate when:

- Subordinates are necessary for implementation and their support and commitment are imperative (Wynn \& Guditus, 1984);

- Creative or divergent thinking is imperative (Wynn \& Guditus, 1984);

- An integrative, high-quality decision is necessary (Wynn \& Guditus, 1984);

- Subordinates have adequate maturity levels and share organizational goals (Yuk1, 1989);

- The manager wants to generate trust with the subordinates (Wynn \& Guditus, 1984).

No further work is planned under this study during the remainder of the grant year.

In Consensus Pacilitator studies, we investigate strategies facilitators may use to foster consensus outcomes of achieved consensus (actual and perceived) and decision quality. In consensus facilitators, we completed a pilot study and a full study of the effects of collaborative versus competitive conflict on consensus. The pilot study assured the adequacy of the manipulation and experimental materials. The results of the full study are reported below.

The main data collection phase investigated the effects of group and individual incentive structures on the development of collaborative versus competitive conflict and the effects of incentive structures and conflict styles on consensus outcomes (actual and perceived decision quality). Half the subjects were in competitive (individual) incentive structure groups and half were in collaborative (group) incentive structure groups.

One hundred-twenty subjects in thirty groups of four viewed the beginning of the movie, Twelve Angry Men. During the movie segment, the jurors are close to agreement on a verdict. Eleven jurors have voted "guilty," but one has noted "not guilty." After 35 minutes and additional deliberation, a second vote is taken. Subjects were asked to predict the sequence in which the jurors changed their votes from "guilty" to "not guilty."

Analyses were conducted as follows. A manipulation check for the effect of individual versus group incentive structure on collaborative conflict and competitive conflict was significant (F-5.72, $p=.018$ and $F-11.73$, p-.0001, respectively). Responses to the competitive conflict 1 cems were not related to actual consensus, but were related to perceived consensus $(r-47, p=.0001)$ and perceived decision quality $(r=-.39$, $\mathrm{p}-.0001)$. Similarly, collaborative conflict responses were not related to actual consensus, but were related to perceived consensus $(r-.54$, $\mathrm{p}=.0001)$ and perceived decision quality $(\mathrm{r}-.66, \mathrm{p}=.0001)$. 
Individual versus group incentives had no effect on actual consensus, but did influence perceived consensus ( $F=24.28, p=.0001$ and perceived decision quality $(\mathrm{F}-23.18, \mathrm{p}=.0001)$.

The study also assessed possible gender effects on consensus outcomes and the possible interaction of gender with incentive structure. Results indicated that significant gender effects occurred for perceptions of collaboration, but not for other measures.

So far, with the exception of the actual consensus measure, we've talked primarily of perceptions. A behavioral analysis was conducted to determine whether there was a relationship between people's perceptions of the group process and their actual verbal behaviors. During the experiment, proctors logged the quantity of all group members' verbal behaviors. Behaviors were classified according to task questions, shared task information, and off-task verbal behavior. Results revealed the difference in amount of verbal behavior due to gender was insignificant. However, incentive structure did contributu to the amount of verial behavior. Groups using competitive conflict had more verbal behavior than groups using collaborative strategies.

Results also revealed that groups exposed to group incentives took ten minutes less than their individual-incentive counterparts in making the decision. When length of group process (time), the average score on the competitive questionnaire, and the average score of the collaborative questionnaire were used to predict several criteria (qualities of task questions, task-related information, and off-task behaviors), only time was a significant predictor. In addition, results suggest that the quantity of people's verbal behaviors is related to perceptions they have influenced the group in a consensus task ( $p-.01)$.

The implications of these findings are 1) group incentives lead to collaboration, while individual incentives lead to competitive conflict, 2) collaborative conflict yields an enhanced perception of decision quality and perceived consensus, while competitive conflict diminishes perceptions of decision quality and consensus. None of the predictor variables appeared to be related to the actual consensus (agreement) measure. This study has been completed.

In Personality Variables and Consensus, preliminary work was begun early in year four. The focus of this line of research pertains to tha effect of an individual's influence in the decisions of others. In particular, we're looking at the effect of certain personality traits on individuals' perceptions of consensus. First, participants completed the Personality Research Form. Using a Lost-on-the Moon exercise, groups of four came to consensus on the correct answer for the moon task. Analyses are under way for this study and will be complete by the end of the fourth grant year. Possible follow-up studies will be completed in the fifth year of the grant.

Presentations and Papers are included in Volume II, Papers, Presentations, and Conferences. 
Consensus Process Studies

\section{Background}

The primary focus of our efforts in year four is on consensus process studies to (1) develop conceptual models of EM and (2) conduct studies in the real-world environment of $\mathrm{EM}$. In addition, we're establishing and maintaining working relationships with the managers and staff in EM.

In establishing working relationships, we continue to be inivolved in EM programmatic activities such as the five-year plan and strategic planning activities. These activities keep our managers involved in the EM program and its changing culture. We continue to initiate and facilitate consensus groups. We've participated in the stakeholders forum for EM, the State and Tribal Government Working Group (STGWG), and the EM program managers' meetings. These groups offer excellent opportunities for us to establish working relationships with the critical EM consensus personnel and enable us to measure various aspects of consensus.

In addition to measuring variables in consensue group meetings, we performed an historical, qualitative analysis of the data available surrounding the STGWG and the five-year plan.

We're developing a survey method to measure consensus on key, programmatic variables involved in EM's interactions with the general public. Two forums may help us gain visibility of key, programmatic variables. First, EM must respond in writing to questions submitted by the public. We'll poll those members of the public who submitted questions to EM. At various levels in EM and for various activities such as the programmatic environmental impact statement process, EM is required to hold public hearings. We'll investig ze methods for measuring key, programmatic variables in or as a result of these types of public hearings.

Accomplishments

The major focus of the consensus processes domain is to design and conduct real-world tests of conceptual models of EM. For consensus process, we're working on nine studies during the fourth grant year. We've added five new studies called: EM complex modeling, EM-40 strategy retreat, transportation analysis, consensus measurement, and $E M$ exhibit. We've completed two studies during this grant year called EM-40 strategy retreat and transportation analysis.

In Management Analysis and Networking, we continued to carefully observe DOE's progress in communicating and promulgating its new culture. We've observed Headquarters, Field Office, Contractor, and off-site participants change.3. Culture is an important variable which can affect consensus. As a result, our understanding of the cultural drivers within DOE becomes important to our understanding of consensus. This study will be completed in year four. 
In Consensus Metings, we continue to study and interact with consensus groups and measure several variables. Our variables are chosen and measured to determine whether or not, in field settings, the meeting of expectations is a critical element in group members' perceptions of decision quality and strength of consensus. We continue to develop and apply instruments in the real world of EM to generate data to complement the fundamental research activities, and to analyze and interpret data. To help support these measurement and analysis functions, we're developing an environment that simulates the information in the EM world realistically enough to enable us to generalize research results. We've begun a survey of members of the public who've submitted questions to determine the effectiveness of the responses provided.

One area of consensus meeting research has been the Stakeholders' Forum. We gained valuable data on inter-group consensus. As indicated in the year-three section, we helped participants elicit and gather comments and developed a report based on these comments. During year four, we are in the process of completing the computer analysis of the collected data. Unfortunately, because of the newness of Stakeholders' Forum, long-term results aren't yet available. However, we are developing a set of analytic tools and methodologies which will enable us to glean some statistical inferences from the existing data sets.

As a result of funding reprioritizing, we're planning only to observe and measure the Spring 1992 Stakeholders' Forum and implement the new questionnaire on the consensus group for the first time. This questionnaire was designed to conduct exploratory research into the relationship between the process components of the meeting and the opinions about DOE. The questionnaire has a reference question (with known results from a national survey) which can be used with Bayesian Methods to estimate the frequency of the responses to the questions.

The design of the new questionnaire was based on the results of the study of the State and Tribal Government Working Group (STGWG) meetings. During year four we continued the analysis of STGWG by using a questionnaire consisting of 16 questions (six on general meeting, six on executive session, four on opinion of DOE). The data analysis revealed that the executive session questions were redundant and did not need to be continued in future questionnaires. In our judgement, the remaining 10 questions fit into four categories: 1) consideration - were participants' opinions given a fair hearing, 2) met expectations - were the goals of the meeting reached, 3) process - satisfaction with the way the meeting was conducted, and 4) DOE trust - how much did participants believe DOE. The answers in each category were found to be so closely related that the average was the best measure for that category's answer.

As a result of funding repriorltizing, we're planning only to observe and collect data on the Fall 1992 STGWG meeting. During this meeting we'1l gain our second set of data input using our recently developed questionnaires. We'll continue to develop and refine the analytical computer models to perform the detailed statistical analysis necessary to detect continued changes in the consensus variables. 
A new research effort under consensus meetings involves ER Technology Information Exchange (TIE) Workshops. This new activity has been added to our grant scope for the second half of year four. We've been asked to research ways to improve DOE's technological information exchange effectiveness. Specifically, this includes researching ways to improve internal communication within the Office of Environmental Restoration (ER) and between ER and EM's Office of Waste Operations and Office of Technology Development. Our laboratory will participate on a Headquarters and Field Area Technology Representatives (HATR/FATR) Steering Group.

As a participant, we will help the HATR/FATR Steering Group to research, design, develop, and facilitate technology information exchange (TIE) workshops. The purpose of the TIE workshops is contained in their theme, "Using Today's Technologies Better." The workshops pioride a "hands on" site-wide technival exchange of information between participants to continue an ongoing process of site-to-site communications and sharing of iessons learned among ER fleld personnel. That is, their purpose is to create and strengthen "TIE's" among sites.

During the remainder of Year four, we'll help the HATR/FATR Steering Group to research, design, develop, and facilitate the second TIE workshop scheduled for May 19-21, in Albuquerque, New Mexico. As facilitators and observers of the TIE workshop, we'll be able to gather data on intra-group (within DOE) consensus building. Following the workshop, we'll assist the HATR/FATR Steering Group with the research and development of a proceedings of the workshop to continue information exchange among the sites.

The application of the TIE concepts we've developed has been expanded to include the Office of Technology Development (OTU) Technical Program Managers' (TPM) Steering Group. This group is in the process of developing TIE concept workshops focused on improving the exchange of information among OTD managers and field personnel.

Another meeting process research effort is Waste Operations Resource Allocation Support System National Workshops (RASS). Funding for extensive MSL involvement in the research and analysis of consensus groups has only been allocated for research on the effectiveness of the Waste Operations Resource Allocation Support System National Workshops (RASS). We'll actively participate in the design, testing, evaluation, and feedback of the development of these workshops and consensus group meetings.

All of these consensus meetings provide a real-world opportunity to test and validate our EM conceptual models.

In Networking for Consensus, United Minerals and Energy, Inc. (UME) conducted extensive research into Consensus Management in support of this grant. These activities were performed under a subcontract to Virginia Tech. In summary, UME conducted extensive research on consensus management literature, including approximately 1,750 books, journals, speeches, articles, and other documentis. From this literature search, approximately 125 were determined to be relevant to our research 
and accordingly, short abstracts were prepared and forwarded to MSL. These will be included in the bibllography of the final report.

We carefully reviewed and assessed various Department of Energy (DOE) waste management policies, procedures, and programs that are currently utilizing outside interest groups for oversight, feedback, and recommendations: The efforts of these groups are documented to determine the extent to which these groups are affecting the Department's directions. Specific groups include the State and Tribal Government Working Group (STGWG), Stakeholder, and others.

We reviewed many of the previousiy prepared draft documents and case histories for potential inclusion into the consensus management final project. We participated in various meetings with staff from MSL to scope the final report.

During the remainder of the grant year, we'11 continue the comprehensive literature search for relevant books, journals, articles, and other documents on consensus management for the bibliography of the final report. It is expected that approximately 375 documents will be reviewed and 60 abstracts will be prepared.

We'll continue to review, evaluate, and refine all previously prepared draft documents including consensus management case histories for inclusion in the final report.

We'11 conduct further analysis and assessment of DOE policies and programs that are currently utilizing outside focus groups for feedback, evaluation, and recommendations. This includes the documentation of the State and Tribal Government Working Group (STGWG).

We' 11 assist in the formulation of draft strategies for a final report. This will include: identification of draft materials to be included, format of report, and case studies and bibliography to be included in the final report.

In EM Complex Modeling, EM has es cablished the goal of cleaning up the current inventory of inactive and surplus facilities by the year 2019. The size and complexity of the clean-up problem is staggering. EM-30 has initiated the Configuration Study, an important element in meeting EM's 30-year cleanup goal. The study intended to answer the question: "What treatment, storage, and disposal facilities (TSD) will be needed, when will they be needed, and where should they be located to process DOE waste over the next 30 years?" There are many important criteria including cost, waste volumes, treatment and disposal technologies used, regulatory issues, and many more. In this project, we'll help EM formulate the problem and develop solution methods.

Neither the problem nor the solution methods are clearly defined. So, EM managers will have to formulate strategies to achieve the clean-up goals that are based on assumptions and "best guesses". Further, these strategies must withstand intense public and regilatory scrutiny. Mason and Mitroff in Challenging Strategic Planning Assumptions state: 
"... complex problems depend on assumptions because it is not humanly possible to know everything of importance about a problem of organized complexity prior to the taking of action.

Most policymakers are unaware of the fact that much of their action rests on assumptions and, moreover, they are unaware of the particular set of assumptions they hold.

Problems of organized complexity ... are ongoing, ill-structured, and generally wicked. The choice of individual courses of action is only a part of the manager's or policymaker's need. More important is the need to achieve insight into the nature of the complexity and to formulate concepts and world views for coping with it. It is the policymaker's thinking process and his or her mind that needs to be supported."

EM managers are developing and using mental models (which are incomplete) of the EM facilities and processes to guide decision-making. These mental models can't be used to achieve consensus on strategies, since assumptions are hidden, possibly even from the EM managers. Unless EM managers can achieve consensus with the public and regulatory agencies about their strategies, the strategies can't be implemented and won't be effective. To improve their ability to build effective strategies and make decisions, models must reflect the global problems facing the managers. Further, formalization of these models will allow managers to explicitly analyze the underlying assumptions and bases so they can be improved and made visible to those with whom EM must achieve consensus. EM Modeling Study includes projects aimed at:

1) establishing the need for specific models,

2) identifying and analyzing existing models, and

3) building new models.

Later. this grant year, we plan to model the EM Complex by first establishing a review panel with expertise in management, public outreach, consensus processes, operations research, systems analysis, and environmental science. Their focus will be on problem formulation. They'11 work with DOE managers to define the problem and the solution criteria. Second, ESL researchers will work with EM-30 managers to develop a model of the EM Complex capable of the needed analysis based on the problem formulation developed by the review panel. This model will be used by EM managers to try out different EM Complex configurations, surface and test assumptions, and make these assumptions and findings available to the public and to DOE oversight groups.

We also plan to develop other models of EM or aspects of EM as our research progresses during the year.

In the EM-40 Strategy Retreat, we pulled from our University resources and teamed with the Virginia Productivity Center under the direction of Dr. D. Scott Sink. The strategy retreat was an excellent opportunity for us to gain a deep understanding of the inter-and intra-office strategic issues confronting EM-40. We interviewed numerous EM-40 managers to 
understand their strategic roles in EM-40's process improvement, organizational goals, and operations. We then facilitated the retreat based on these interviews to determine if we could improve and expedite consensus among these managers on these volatile issues. A report of output was produced by the Virginia Productivity Center describing the results of the retreat. This study was begun, completed, and closed in year four.

In the EM Exhiblt study we continued to examine how the public interacts with the EM Exhibit. We've designed a variety of outreach materials and measured how the public responds to these materials and the exhibit itself. Because measurement of the Exhibit's effectiveness is a low priority study, we've videotaped public interactions with the exhibit for future review and analysis to minimize travel costs associated with following the exhibit around. We're also preparing and testing an instruction manual to enabie the operators of the exhibit to present a consistent and effective approach in communicating with the public. This study continues through year four.

In the Transportation Analysis study we applied one of our measures of consensus effectiveness to a meeting of local governments. Eighteen local government officials and four DOE officlals met under our design of a consensus facilitation workshop. They discussed issues ranging from local government incident authority, to outreach programs, to federal support for developing more cost effective public notification. We designed the consensus processes used in this workshop and measured the effectiveness of the elementary consensus variables we've developed thus far. The participants unanimously agreed that this type of workshop is an excellent way for DOE to exchange information, concerns, and ideals, with local governments. This study was begun, completed, and closed in year four.

In Consensus Measurement, we focused on development of consensus measurement tools. We've developed a survey which we've pilot-tested and modified. We have analyzed the data using descriptive statistics to describe prevailing opinion of group members.

We're investigating cluster analysis and multi-dimensional scaling as possible methods for assessing consensus. These efforts are in the exploratory stage and will be more fully reported in next year's technical progress report.

Presentations and Papers are included in Volume II, Papers, Presentations, and Conferences. 


\section{Background}

In the past, we used the Information Systems Studies to develop relationships with DOE employees at HQ and in the field and to study the types and amounts of data and information avallable in EM and its environment. We wanted to look at what data and information was avallable, its accuracy, timeliness, consistency, and accessibility by EM and people external to EM. These variables of data and information affect the ability of EM to achieve consensus within EM and with groups external to EM. In many cases, as we analyzed EM data, we made recommendations of changes to be made by EM to resolve deficiencies resulting from missing, inconsistent, or inaccessible data and information. In some cases we produced reports to resolve data deficiencies for EM.

In addition to maintaining working relationships and studying EM's data and information environment, we've begun to focus on developing an EM research environment. The goal of the EM laboratory environment is to develop information in our laboratory that simulates the information in the real-world EM realistically enough to enable us to generalize results to the real-world of EM.

In year four, we've begun to use fundamental and consensus process research to develop EM conceptual models and test them in a research environment. An example of such a development effort would be to take a conceptual model of the key programmatic variables for EM and develop situation scenarios in a gaming environment in our laboratory. Such a game could be computer-based to allow rapid changes of parameters. We could then easily design a number of experiments to vary parameters and measure the results of various subjects interacting with the EM environment. The results of testing our lab models will be applied in Consensus Process Studies in real EM environments and in fundamental research to support or contradict 11terature findings.

\section{Accomplishments}

In Information Systems, we're working on four studies during the fourth grant year. We've added two new studies this year called testing the EM booklet and testing the EM fact sheets.

In EM Hazardous and Sanftary Waste Program Background Reports, MSL was asked to produce these background reports to serve as reference material to support the EM Office of Waste Operations in formulating two new HQ programs, the Hazardous Waste Program and the Sanitary Waste Program. EM needed information about hazardous and sanitary waste activities and regulations throughout DOE to determine the scope of these programs, establish program baselines, and develop program objectives and strategies for accomplishing the objectives. We agreed to review the relevant background materials and prepare comprehensive background reports for these waste programs as part of our consensus research so we could determine the types of information needed to establish these EM 
waste programs, and determine the availability and consistency of waste information throughout the DOE complex.

The Hazardous Waste Program and Sanitary Waste Program Background Reports were built from a compilation of materials from available publications created by or pertinent to DOE. The main source of data for the background reports was the draft 1991 site-Specific Plans. A myriad of "Information bites" were extracted from these plans and organized by site and activity to serve as the foundation for the detailed background reports. MSL combined this site-specific foundation with information obtained from the EM Five-Year Plan, EPA regulations, DOE Orders, the Waste Information Network databases, and other related documents. The final drafts of these reports were completed in midMarch.

We found substantial deficiencies in existing waste data for these programs. Specifically, waste data volumes throughout its life-cycle were not available for some locations. Where waste data was available through several sources, often the waste data volumes did not agree. In some cases the way waste was characterized and quantified on various reports made it impossible to cross mcheck the data from one source to another. In addition, there was a deficiency of data regarding future trends in regulatory compliance requirements and future issues. A consistent vision of what the $E M$ environment will be like in the future is important in establishing objectives that are proactive rather than reactive. Data on the waste budget, existing regulations, and waste activities was generally avallable and in good form.

In Testing the EM Booklet, MSL, through another funding vehicle, performed DP Intern training for 17 DOE interns during the fall semester of 1991. Through this grant, we wanted to evaluate the EM Booklet as text material to teach the interns about EM. This would aid us and DOE in understanding the usefulness of the EM Booklet as an education tool. EM has stated that the public must become better educated about EM and its programs so it can better work with the public to accomplish DOE's mission. As such, the information transfer of public literature such as the EM Booklet affects DOE's ability to reach consensus with the public on its program.

The interns were future DOE employees-college-educated professionals with a vested interest in learning the material presented to them. The training program introduced each office within DOE, including a session on EM. All the students were given a pre-test to determine their knowledge before the EM session began. Some students received a pretest on information unrelated to EM but included in previous lectures; the others received a pre-test exclusively on EM-related information. The instructor presented a three hour lecture on EM that included information not covered in the EM Booklet. The instructor did not know an evaluation test was being conducted. The interns received the EM Booklet during the session on EM and were told to read it.

We conducted a post-test one week after the interns received the EM Booklet and attended the lecture. The EM pre- and post-tests were designed to ask. the same questions worded differently and in a random 
order. The test questions included specific material the readers should learn from the EM Booklet. At least 258 of the test questions were on material from the session that wasn't covered in the EM Booklet. This allowed us to determine the effect of previous knowledge about DOE activities on learning from the EM Booklet. Of the 17 interns, 14 turned in both pre- and post-tests.

We specifically looked at three research questions. (1) Did we bias the interns' post-test scores by giving them a pre-test (in other words the pre-test did not clue the interns in on what to study)? We found that the pre-test did not affect the scores on the post-test. (2) Did the lecture and the EM Booklet teach the interns material they did not already know? The test scores were analyzed using the Wilcoxon Signed Ranks test. The post-test scores were significantly higher than the pre-test scores $(p-0.005)$. We concluded that the lecture and the EM Booklet taught the interns materials they did not already know. The sample average improvement in their test scores was 20\%. (3) Did the lecture affect how much the interns learned from the EM Booklet? The results showed that the lecture did not affect test scores and was not an effective tool for reinforcing the EM Booklet (p-.20).

In Testing the EM Fact Sheets, our objective was to explore the effectiveness of the fact sheets as teaching tools, evaluate whether exposure time affected learning, and test whether attitude about nuclear power and the transportation of nuclear waste affected knowledge acquisition. EM has stated the public must become better educated about EM and its programs so they can better work with them to reach consensus about how EM should accomplish its mission.

We used a sample of 15 undergraduate college students who had completed one or more semesters of college and who did not have a vested interest in learning about EM. We tested four of the 35 existing EM Fact Sheets: Resource Conservation and Recovery Act, Environmental Restoration Activities at Feed Materials Production Center, Waste Management Activities at Idaho Field Office, and Radioactive Waste. We measured their baseline knowledge about EM with a pre-test. The test questions reflected specific material the readers should learn from the four EM Fact Sheets. The post-test asked the same questions differently and in random order. We also included opinion questions about radioactive waste and nuclear power.

The test was set up in a complete randomized block design with each block receiving one of two treatments. Group One was given five minutes to read each fact sheet in a mock-exhibit environment (people talking, exhibit materials on the walls, TV, displays, etc.). They received a post-test four or five days later. Group Two took the four EM Fact Sheets home and were instructed to read them twice for ten minutes each a few days apart. They received a post-test four or five days later.

We answered five questions with this test. The first question was, did the two groups have different post-test scores (in other words did time exposure affect test scores)? There was no difference in test scores based on length of exposure time for this audience. Second, did the EM Fact Sheets teach the students information they did not already know? 
We found there was a 248 increase in test scores from the pre- to the post-test for both groups combined ( $p-.005)$. The pre-test on average was 198 and the post-test on average was 438. Third, did attitude affect test scores? Attltude toward nuclear power or waste transportation didn't appear to affect test scores. However, it appears that access to information about these topics did not negatively impact this audience's opinions about radioactivity or nuclear power. Fourth, were test scores higher for different topic fact sheets? None of the four fact sheets was more effective than the others ( $p=.3)$. Fifth, were more questions answered correctly when based on information at the beginning and end of the fact sheet than the middle? There was no discernible pattern of knowledge retention for different parts of the fact sheet.

Presentations and Papers are included in Volume II, Papers, Presentations, and Conferences. 


\section{STUDY RESULTS: MILESTONE LISTINGS}

For an effort as large as our grant, we use a numbering mechanism to keep track of everything we do. We also define physical evidence to track our progress. Sometimes we cause interim activities to end in physical evidence just to track and evaluate progress. This physical evidence is the tangible results related to the milestones. Figures 9 , 10,11 , and 12 are gante charts that not only show the scheduling for the studies we're working on but aiso include the milestones we've identified to monitor our progress and show completions. The time lines for the gantt charts are divided into quarters of the year. These are contract year quarters. Therefore the first quarter of the first grant year is for October through December 1988. Figures 9, 10, 11, and 12 provide representative samples. Complete milestone lists are provided to each study manager quarterly in the quarterly reports.

To follow Figures $9,10,11$, and 12 and to recall the studies described in our discussion of each research domain, review the following study 1ist. The numbers identify each study. The numbers were assigned as we approved scoping documents for each study. Therefore, the number sequence is chronological within each major topical area. For our own management practice, we use these numbers to identify each task and milestone within every study. We also use the numbering scheme to track ciosts so we can plan studies, allocate resources, track physical evidence of progress, and determine actual costs. Within this numbering scheme, we've identified a "study" for managing and administering the grant (what we've just been talking about) and a "study" for procuring the equipment.

The milestones for Direct Thesis and Dissertation Activities (101 numbers) are papers, presentations, theses, and dissertation completions. Since all our papers and presentations are documented in Volume II, we chose not to graph them as milestones. 
Studies and substudies as of the First Annual Performance Report -21 studies. (The parenthetical words refer to effort areas from Figure 2.)

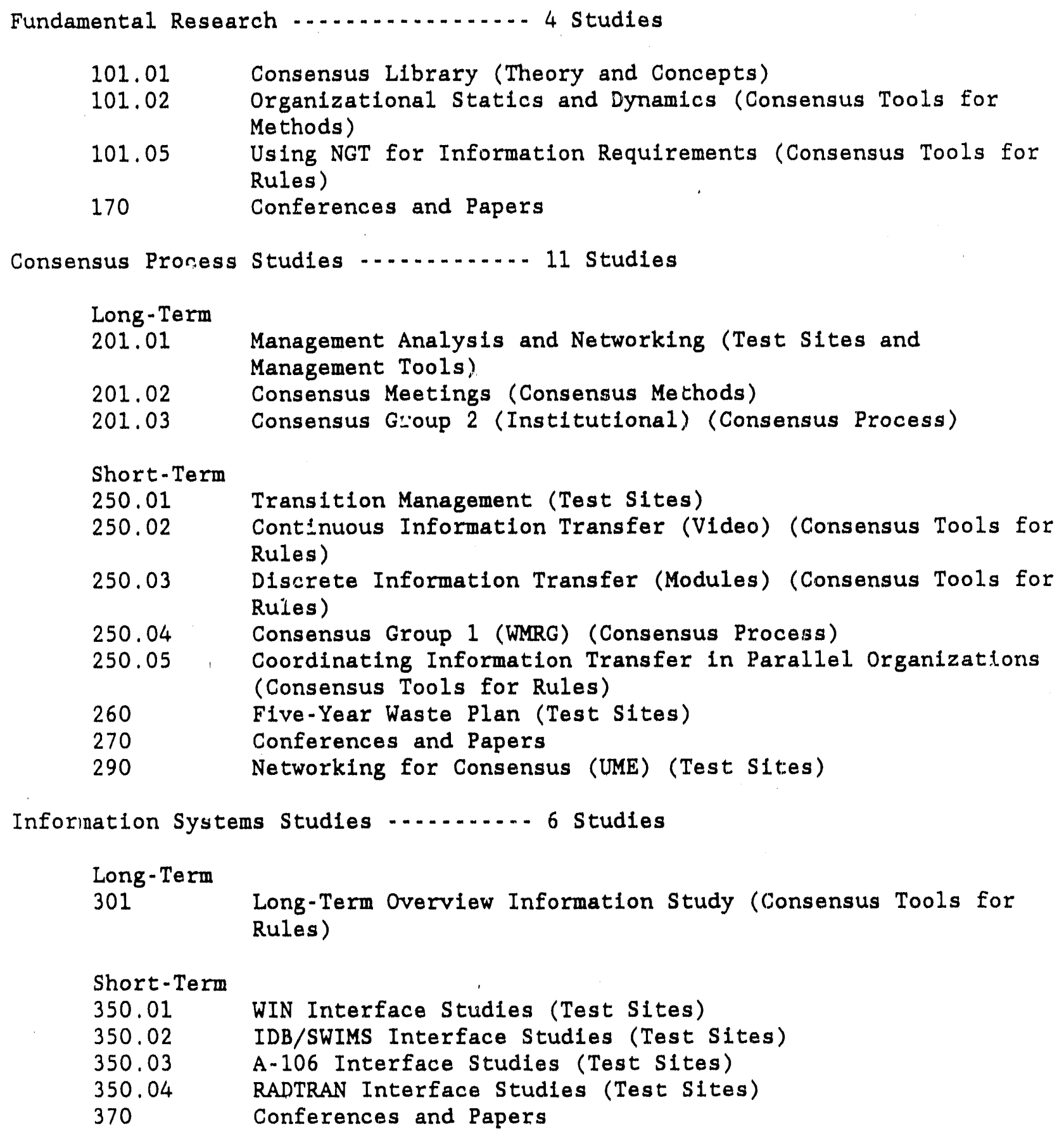


Studies and substudies as of the end of the first grant year - 33 studies $=21$ studies from First Annual Performance Report plus 12 new stiddies. (The parenthetical words refer to effort areas from Figure 2.)

Fundamental Research ........................... Studies

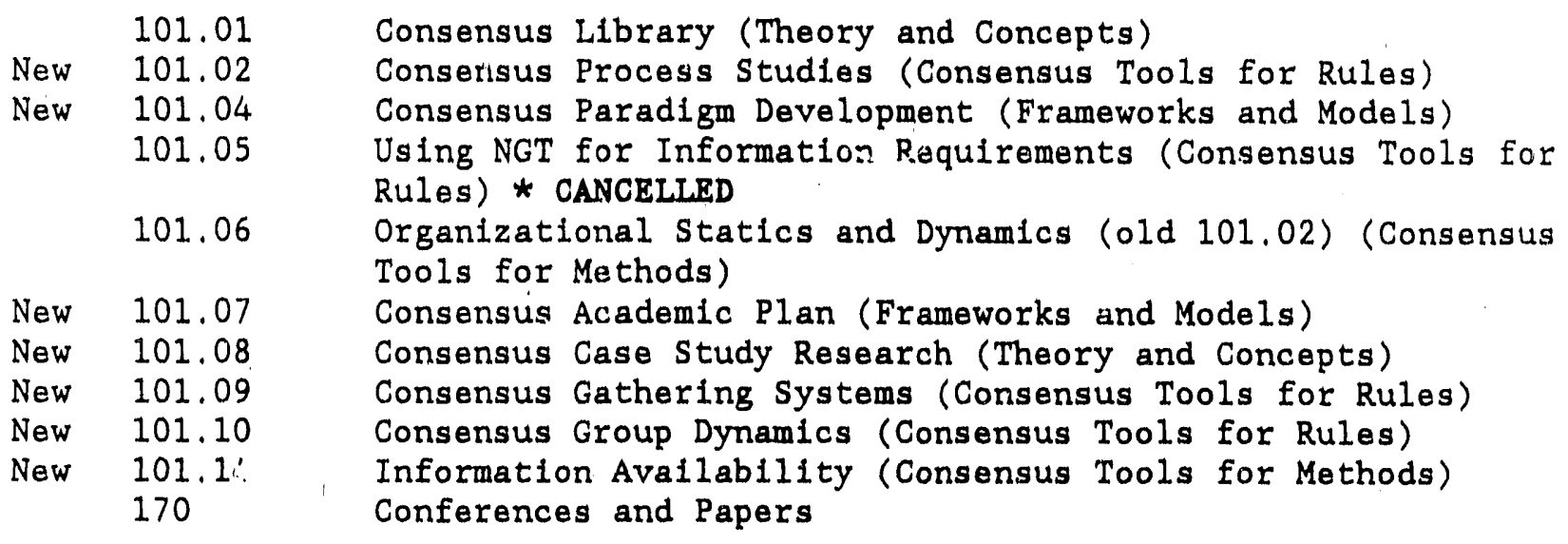

Consensus Process ztudies .................... Studies

Long - Term 201.01

201.02

201.03

New 201.XX

Short-Term

250.01

250.02

250.03

250.04

250.05

New 250.06

New 250.07

260

New 261.01

270

290
Management Analysis and Networking (Test Sites and

Management Tools)

Consensus Meetings (Consensus Methods)

Consensus Group 2 (Institutional) (Consensus Process)

Consensus Group 3 (Interagency) (Consensus Process)

Transition Management (Test Sites)

Continuous Information Transfe: (Video) (Consensus Tools for

Rules)

Discrete Information Transfer (Modules) (Consensus Tools for Rules)

Consensus Group 1 (WMRG) (Consensus Process)

Coordinating Information Transfer in Parallel Organizations (Consensus Tools for Rules)

Nuclear Waste Transportation (Consensus Methods)

Regulatory Consensus Research (Land Disposal Restrictions)

(Consensus 'rools for Rules)

Five-Year Waste Plan (Test Sites)

Five-Year Research and Development (Test Sites)

Conferences and Papers

Networking for Consensus (UME) (Test Sites) 


\section{LISTING OF GRANT STUDIES}

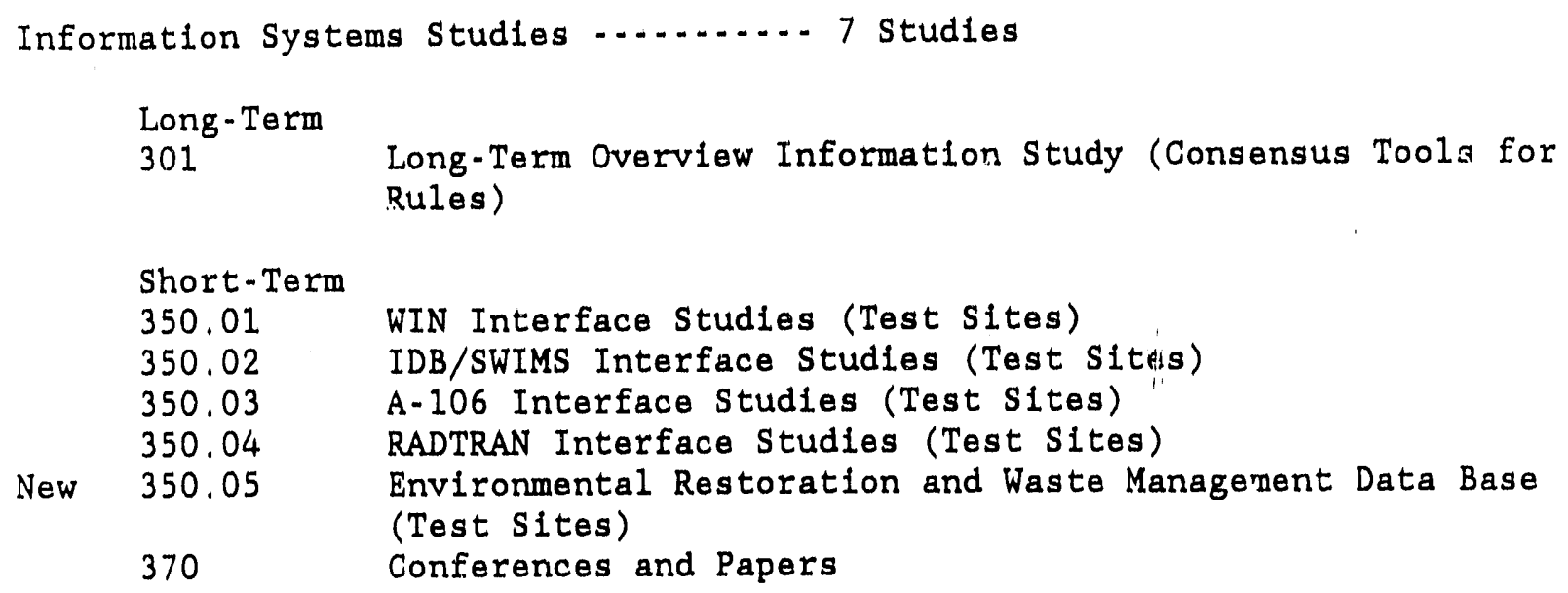

WIN Interface Studies (Test Sites) 


\section{LISTING OF GRANT STUDIES}

Studies and substudies as of the Second Annual Performance Report - 40 studies - 33 studies from end of first grant year, plus eight new studies, minus one study cancelled. (The parenthetical words refer to effort areas from Figure 2.)

Fundaluental Research ......................... Studies

101.01 Consensus Library (Theory and Concepts)

101.02 Consensus Process Studies (Consensus Tools for Rules)

101.04 Consensus Paradigm Development (Frameworks and Models)

101.06 Organizational Statics and Dynamics (old 101.02) (Consensus Tools for Methods)

101.07 Consensus Academic Plan (Frameworks and Mode1s)

101.08 Consensus Case Study Research (Theory and Concepts)

101.09 Consensus Gathering Systems (Consensus Tools for Rules)

101.10 Consensus Group Dynamics (Consensus Tools for Rules)

New 101.11

Consensus Techniques (Consensus Methods)

New 101.12

Consensus Facilitators (Consensus Tools for Rules)

New 101.13

Consensus Scenario Generation (Consensus Tools for Methods)

101.14

Information Availability (Consensus Tools for Methods)

170

Conferences and Papers

Consensus Process Studies ................. 18 Studies

Long - Term

201.01

201.02

201.03

201. XX

Short-Term

250.01

250.02

250.03

250.04

250.05

250.06

250.07

New 250.08

260

261.01
Management Analysis and Networking (Test Sites and Management Tools)

Consensus Meetings (Consensus Methods)

Consensus Group 2 (Institutional) (Consensus Process)

Consensus Group 3 (Interagency) (Consensus Process)

Transition Management (Test Sites)

Continuous Information Transfer (Video) (Consensus Tools for Rules)

Discrete Information Transfer (Modules) (Consensus Tools for Rules)

Consensus Group 1 (WMRG) (Consensus Process)

Coordinating Information Transfer in Parallel Organizations

(Consensus Tools for Rules) * COMPLETED

Nuclear Waste Transportation (Consensus Methods)

Regulatory Consensus Research (Land Disposal Restrictions)

(Consensus Tools for Rules)

Site Specific Plans (Community Relations) (Test Sites)

Five-Year Waste Plan (Test Sites) * COMPLETED

Five-Year Research and Development (Test Sites) * COMPLETED 
LISTING OF GRANT STUDIES

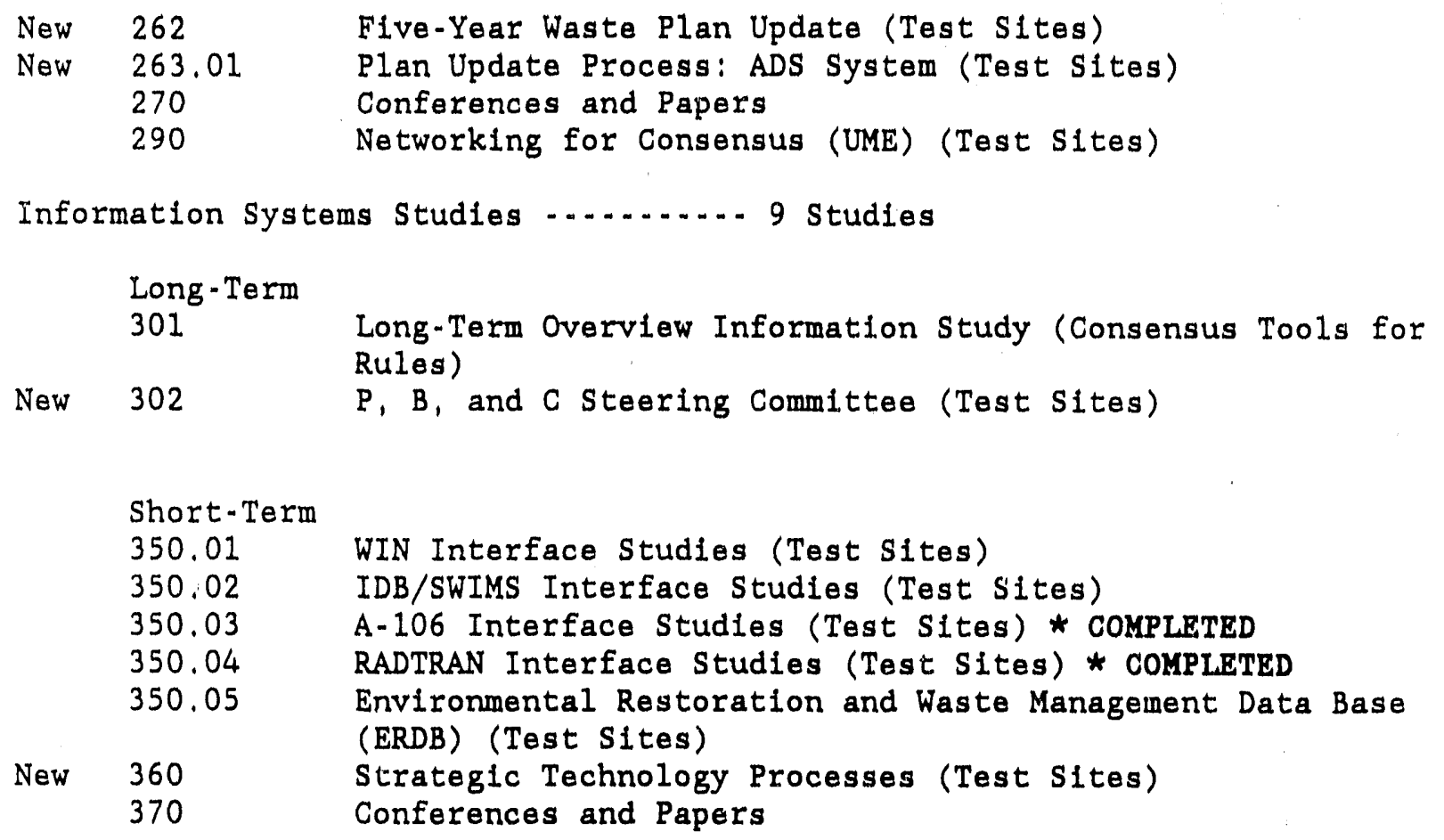


Studies and substudies as of the end of the second grant year - 37 studies = 40 studies from the Second Annual Performance Report, plus 2 new studies, minus five completed. (The parenthetical words refer to effort areas from Figure 2.)

Fundamental Research $\ldots . . . . . . . .13$ Studies

101.01 Consensus Library (Theory and Concepts)

101.02 Consensus Process Studies (Consensus Tools for Rules)

101.04 Consensus Paradigm Development (Frameworks and Models)

101.06 Organizational Statics and Dynamics (old 101.02) (Consensus Tools for Methods)

101.07 Consensus Academic Plan (Frameworks and Models)

101.08 Consensus Case Study Research (Theory and Concepts)

101.09 Consensus Gathering Systems (Consensus Tools for Rules)

101.10 Consensus Group Dynamics (Consensus Tools for Rules)

101.11

Consensus Techniques (Consensus Methods)

101.12

Consensus Facilitators (Consensus Tools for Rules)

101.13

Consensus Scenario Generation (Consensus Tools for Methods)

101.14

Information Availability (Consensus Tools for Methods)

170

Presentations and Papers

Consensus Process Studies .............. 17 Studies

Long - Term

201.01

201.02

201.03

201.XX

Short-Term

250.01

250.02

250.03

250.04

250.06

250.07
Management Analysis and Networking (Test Sites and Management Tools)

Consensus Meetings (Consensus Methods)

Consensus Group 2 (Institutional) (Consensus Process) *

COMPLETED

Consensus Group 3 (Interagency) (Consensus Process) * COMPLETED

EM Command Briefs (Test Sites) (old Transition Management) Continuous Information Transfer (Video) (Consensus Tools for Rules)

Discrete Information Transfer (Modules) (Consensus Tools for Rules) * COMPLETED

Consensus Group 1 (EMRG) (Consensus Process) * COMPLETED Nuclear Waste Transportation (Consensus Methods) * COMPLETED Regulatory Consensus Research (Land Disposal Restrictions) (Consensus Tools for Rules) 


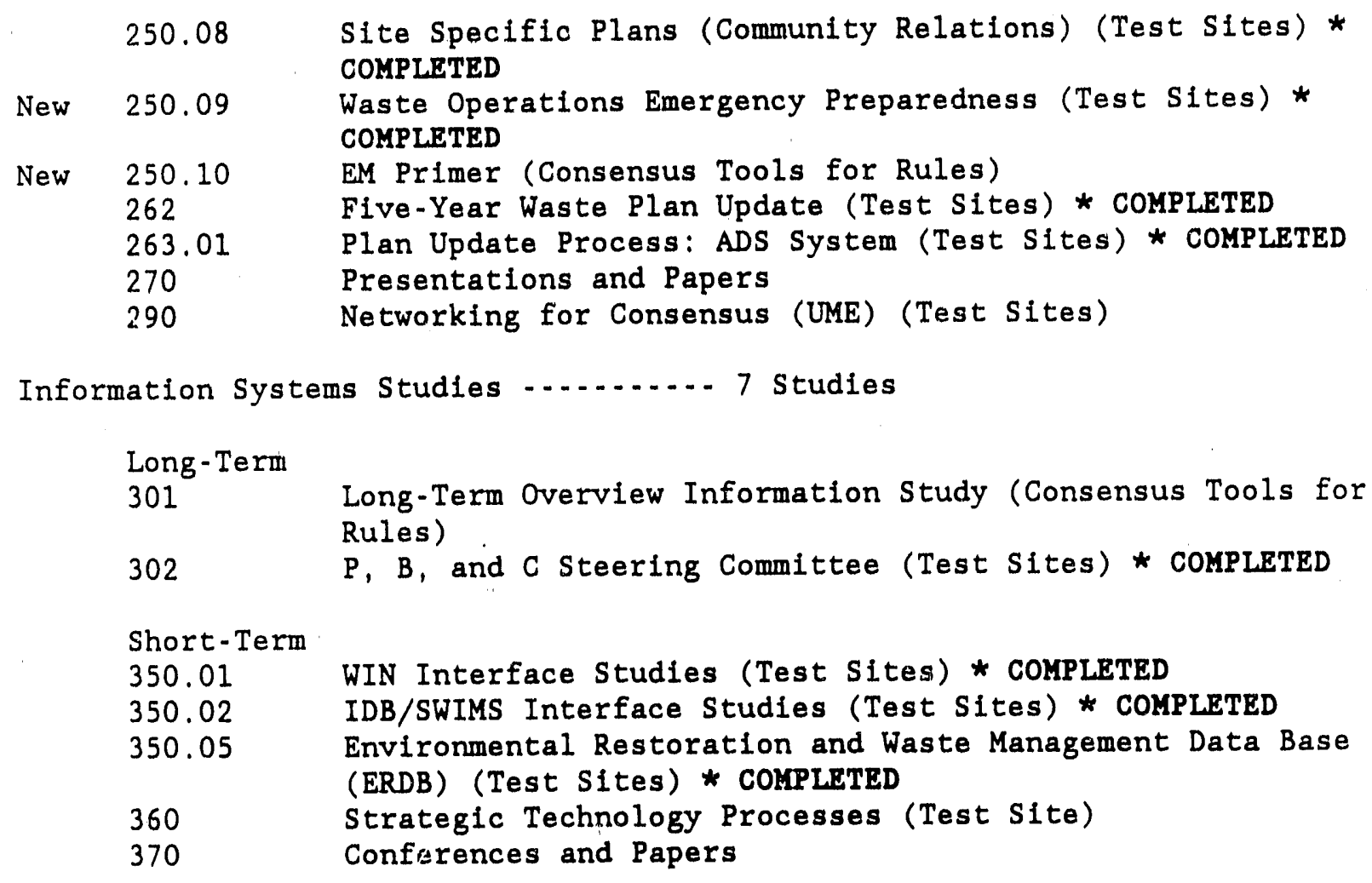


LISTING OF GRANT STUDIES

Studies and substudies as of the Third Annual Performance Report - 27 studies - 37 studies from the end of the second grant year, plus 3 new studies, minus thirteen completed. (The parenthetical words refer to effort areas from Figure 2.)

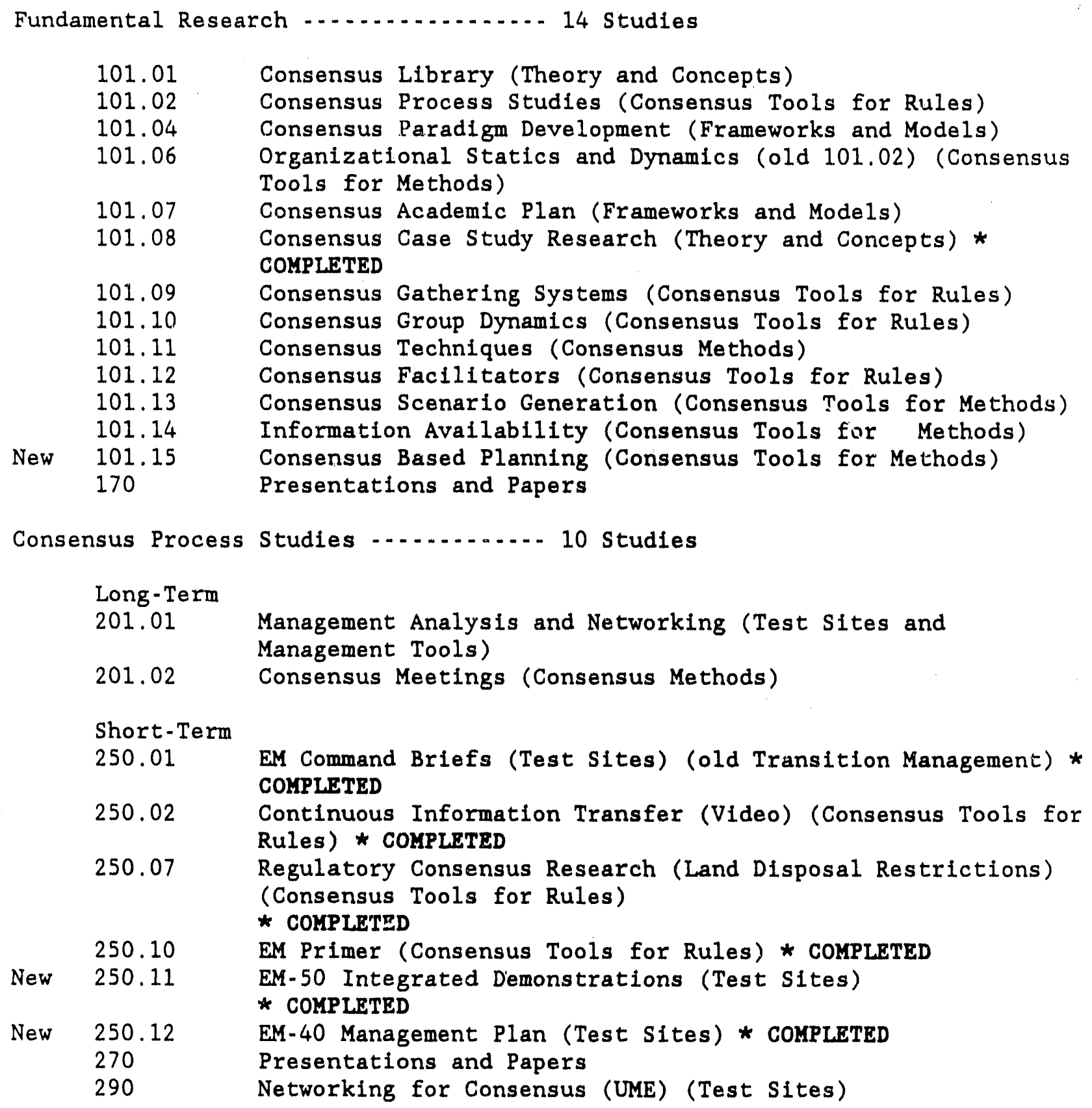


LISTING OF GRANT STUDIES

\author{
Information Systems Studies ......... 3 studies \\ Long - Term \\ 301 \\ Long-Term Overview Information Study (Consensus Tools for \\ Short-Term \\ 360 \\ 370 \\ Rules) \\ Strategic Technology Processes (Test Site) \\ Presentations and Papers
}




\section{LISTING OF GRANT STUDIES}

Studies and substudies as of the end of the third grant year -25 studies -27 studies from the Third Annual Performance Report, plus 5 new studies, minus 10 completed. (The parenthetical words refer to effort areas from Figure 2.)

Fundamental Research ........................ 14 Studies

101.01 Consensus Library (Theory and Concepts)

101.02 Consensus Process Studies (Consensus Tools for Rules) (moved

New 101.03

to Consensus Process Studies applied research section)

101.04

101.06

Environmental Trilogy (Frameworks and Models)

Consensus Paradigm Development (Frameworks and Models)

Organizational Statics and Dynamics (o1d 101.02) (Consensus Tools for Methods) * COMPLETED

101.07

Consensus Academic Plan (Frameworks and Models)

101.09

Consensus Gathering Systems (Consensus Tools for Rules)

* COMPLETED

101.10

101.11

Consensus Group Dynamics (Consensus Tools for Rules)

101.12

Consensus Techniques (Consensus Methods)

101,13

Consensus Facilitators (Consensus Tools for Rules)

Consensus Scenario Generation (Consensus Tools for Methods)

101.14 Information Availability (Consensus Tools for Methods)

* COMPLETED * COMPLETED

101.15 Consensus Based Planning (Consensus Tools for Methods)

* COMPLETED

170 Presentations and Papers

Consensus Process Studies ............ 4 Studies

Long - Term

201.01

Management Analysis and Networking (Test Sites and

Management Tools)

201.02

Consensus Meetings (Consensus Methods)

Short-Term

270

290

Presentations and Papers

Networking for Consensus (UME) (Test Sites)

Information Systems Studies ......... 7 Studies

Long - l'erm

301

Long-Term Overview Information Study (Consensus Tools for Rules ) * COMPLETED

Short-Term

360

New $\quad 530$

New 540

New 610
Strategic Technology Processes (Test Site) * COMPLETED Five-Year Plan Executive Summary Anlaysis (Consensus Tools for Methods) * COMPLETED

MSLTRAK (Consensus Tools for Methods) * COMPLETED

EM Hazardous and Sanitary Waste Program Background Reports 
New $\quad 710$

370
WMIS Conceptual Design (Frameworks and Mode1s) * COMPLETED Presentations and Papers 
Studies and substudies as of the Fourth Annual Performance Report -22 studies - 25 studies from the end of the third grant year, plus 8 new studies, minus 5 completed. (The parenthetical words refer to effort areas from Figure 2.)

Fundamental Research ............. 9 studias

\begin{tabular}{|c|c|c|}
\hline & 101.01 & Consensus Library (Theory and Concepts) \\
\hline & 101.03 & Environmental Trilogy (Frameworks and Models) * COMPLETED \\
\hline & 101.04 & Consensus Paradigm Development (Frameworks and Models) \\
\hline & 101.07 & Consensus Academic Plan (Frameworks and Models) \\
\hline & 101.10 & Consensus Group Dynamics (Consensus Tools for Rules) \\
\hline & & $\begin{array}{l}\text { Consensus Techniques (Consensus Methods) } \\
\text { * COMPLETED }\end{array}$ \\
\hline & 101.12 & $\begin{array}{l}\text { Consensus Facilitators (Consensus Tools for Rules) } \\
\text { * COMPLETED }\end{array}$ \\
\hline New & 101.16 & $\begin{array}{l}\text { Personality Variables and Consensus (Consensus Tools for } \\
\text { Rules) }\end{array}$ \\
\hline & 170 & Presentations and Papers \\
\hline ons & nsus Process & Studies ........... 9 Studies \\
\hline & $\begin{array}{l}\text { Long - Term } \\
201.01\end{array}$ & $\begin{array}{l}\text { Management Analysis and Networking (Test Sites and } \\
\text { Management Tools) }\end{array}$ \\
\hline & 201.02 & Consensus Meetings (Consensus Methods) \\
\hline & Sho: & \\
\hline & 290 & Networking for Consensus (UME) (Test Sites) \\
\hline ew & 510 & EM Complex Modeling (Frameworks and Models) \\
\hline ew & 640. & $\begin{array}{l}\text { EM-40 Strategy Retreat (Consensus Tools for Methods) } \\
\text { * COMPLETED }\end{array}$ \\
\hline ew & 670 & EM Exhibit (Consensus Tools for Methods) \\
\hline ew & 680 & $\begin{array}{l}\text { Transportation Anlaysis (Consensus Tools for Methods) } \\
\text { * COMPLETED }\end{array}$ \\
\hline New & $\begin{array}{l}680 \\
270\end{array}$ & $\begin{array}{l}\text { Consensus Measurement (Consensus Tools for Rules) } \\
\text { Presentations and Papers }\end{array}$ \\
\hline Info & nation System & ns Studies .... \\
\hline $\begin{array}{l}\text { ew } \\
\text { ew }\end{array}$ & $\begin{array}{l}\text { Short-Term } \\
610 \\
680 \\
680 \\
370\end{array}$ & $\begin{array}{l}\text { EM Hazardous and Sanitary Waste Program Background Reports } \\
\text { Testing the EM Booklet (Consensus Tools for Methods) } \\
\text { Testing the EM Fact Sheets (Consensus Tools for Methods) } \\
\text { Presentations and Papers }\end{array}$ \\
\hline
\end{tabular}


First Year Study Results

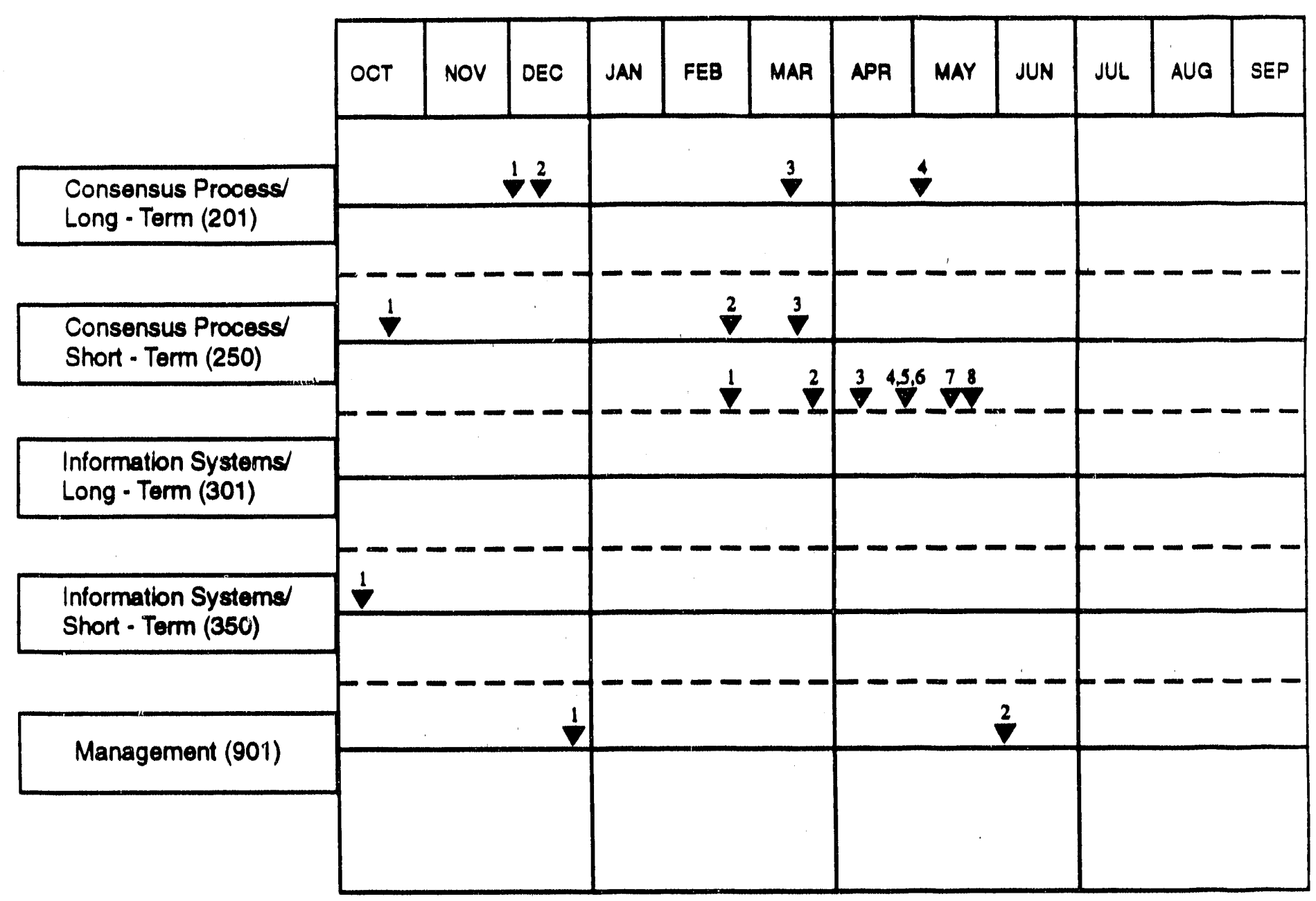

Figure 9. This figure shows our progress toward accomplishing research objectives within each domain during the first year. 
Second Year Study Results

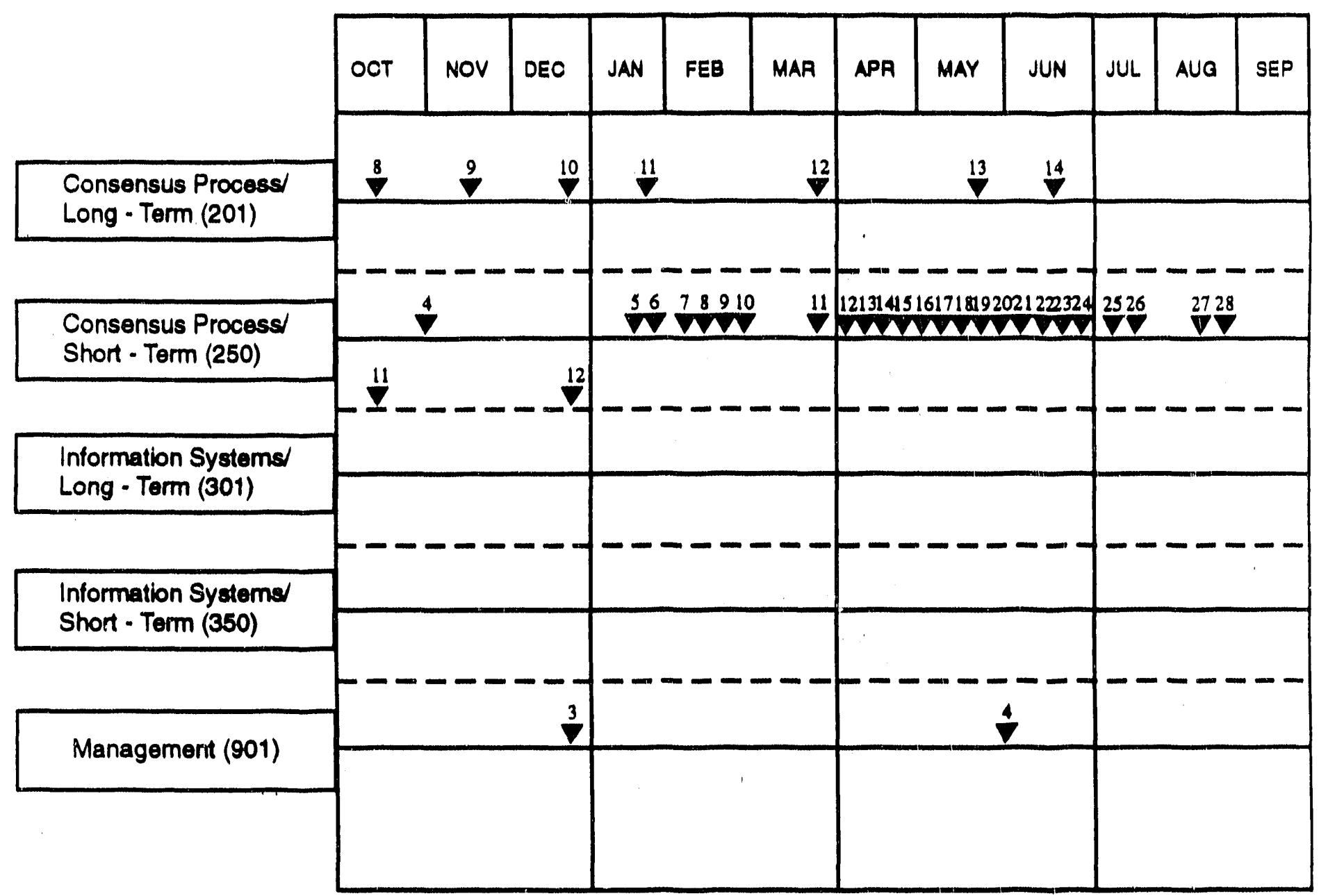

Figure 10. This figure shows our progress toward accomplishing research objectives within each domain during the second year. 
Third Year Study Results

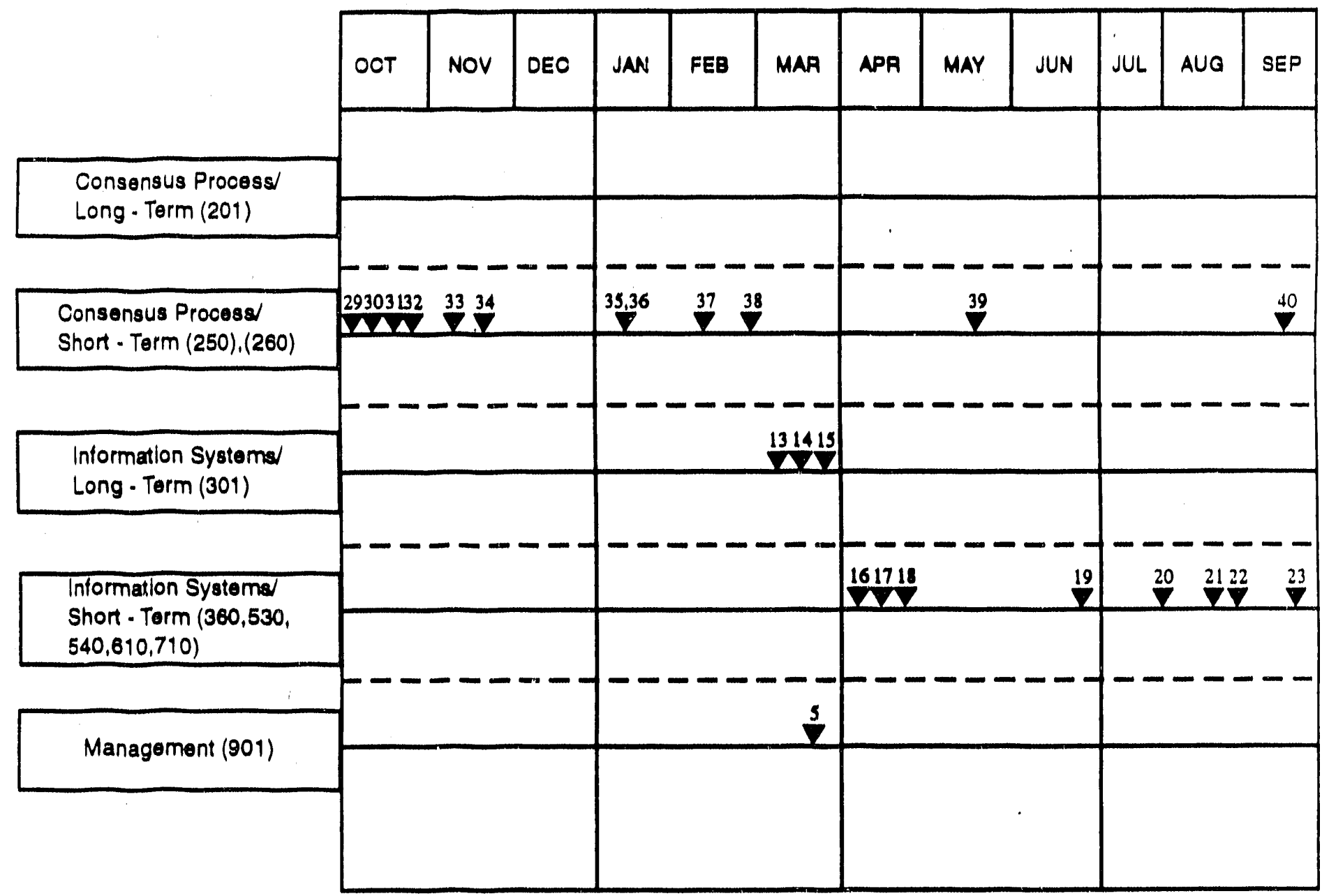

Figure 11. This figure shows our progress toward accomplishing research objectives within each domain during the third year. 


\section{Fourth Year Study Results}

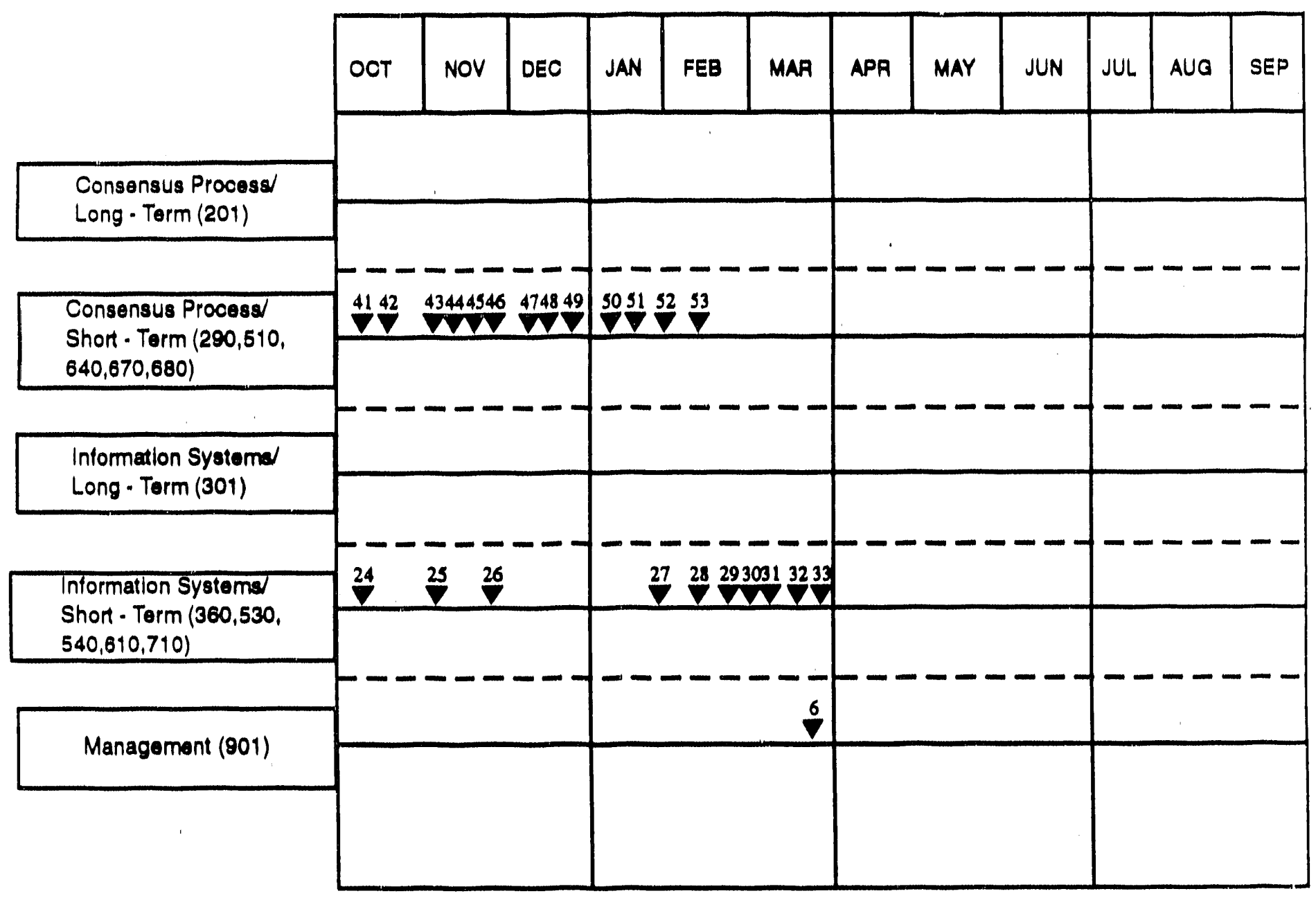

Figure 12. Th1s figure shows our progress toward accomplishing research objectives within each domain during the fourth year. 


\section{FIRST YEAR STUDY RESULTS: KEY TO FIGURE 9}

\section{CONSENSUS PROCESS/LONG-TERY (201)}

1. Facilitate Environmental Restoration (EM-40) program manager's meeting (Denver III) - $12 / 09 / 88$

2 - Deliver report on Denver III - $\underline{12 / 16 / 88}$

3 - Facilitate EM-40 program manager's meeting (Augusta) - $3 / 15-16 / 89$

4 - Deliver letter report on cost assessment team meeting, Las Vegas - 5/02/89

5 - Attend State \& Tribal Government Working Group (STGWG) $I / 10-11 / 89$

6 - Deliver EM-40 program manager's information workbook in Idaho - $7 / 10-11 / 89$

7 - Facilitate EM-40 program manager's meeting (Idaho)$\underline{8 / 1-2 / 89}$

CONSENSUS PROCESS/SHORT - TERK (250)

1 - Deliver results of transition fact sheet brainstorming sesston - $10 / 20 / 88$

2 - Deliver Experimental Consensus Tool for Continuous Information Transfer: Defense Waste and Transportation Management (DWTM) Video - "DWTM - Meeting the Challenge" - $\underline{2 / 23 / 89}$

3 - Deliver Experimental Consensus Tool for Discrete Information Transfer: DWTM Modular Briefing - 3/09/89

\section{INEORMATION SYSTEMS/LONG -TERY (301)}

1 - Deliver Management Systems Laboratories (MSL)/DWTM study report on preliminary operations office findings - $2 / 28 / 89$

2 - Deliver analysis of Low-Level Waste (LLW) manifest requirements - $3 / 22 / 89$

3 - Deliver Information Systems Study (ISS) project update - $4 / 14 / 89$

4 - Deliver DWTM Headquarters (HQ) waste information requirements document planned outlire - $5 / 9 / 89$

5 - Deliver ISS field visits - general findings - $5 / 9 / 89$

6 - Deliver ISS HQ interviews - study findings to date - $5 / 9 / 89$

7 - Deliver ISS briefing: Scope, Progress, and Plan - 5/12/89

8 - Deliver hazardous waste reference study information package - 5/16/89 
9 - Review draft of the ISS survey and data input forms - $8 / 08 / 89$

10 - Deliver Information systems Survey, Volume 1, Waste Tracking System $9 / 30 / 89$

\section{INFORMATION SYSTEMS/SHORT-TERY (350)}

1 - Deliver RADTRAN initial findings - 10/13/88

2 - Deliver systems description and data input forms for hazardous and mixed waste - 7/06/89

3 - Deliver LLW tracking system descriptions - $1 / 12 / 89$

4 - Deliver data input forms for high- and low-level waste for Oak Ridge and Nevada - 8/22/89

\section{MANAGEMENT (901)}

1 - Deliver first-year program plan - 12/29/88

2 - Deliver annual performance report for 10/88 - 9/89: "Research and Development of Methods and Tools for Achieving and Maintaining Consensus Processes in the Face of Change Within and Among Government Oversight Agencies" - 6/2/89 


\section{SECOND YEAR STUDY RESULTS: KEY TO FIGURE 10}

\section{CONSENSUS PROCESS/LONG-TERY (201)}

8 - Deliver EM-40 program manager's information workbook in Sacramento $\underline{10 / 15 / 89}$

9 - Facilitate EM-40 program manager's Sacramento meeting - 11/17/89

10 - Deliver EM-40 program manager's final meeting report in Sacramento $12 / 23 / 89$

11 - Deliver Waste Management summit fact sheet - 1/23/90

12 - Attend the State and Tribal Government Working Group - $3 / 30 / 90$

13 - Facilitate the ER Program Managers' Meeting in Santa Fe - $5 / 23 / 90$

14 - Send out report on ER Program Managers' Santa Fe meeting and discussion $6 / 25 / 90$

\section{CONSENSUS PROCESS/SHORT-TERY $(250) \cdot(260)$}

4 - Deliver information on site specific plans, Section 10 - Interactions to Gale Turi - 11/1-2/89

5 - Deliver suggested outline for Environmental Restoration and Waste Management (EM) site specific plans - 1/20/90

6. Deliver draft EM Information briefings for Waste Operations (WO) and EM-40 $-1 / 29 / 90$

7 - Deliver EM-40 information briefing, Version $1-\underline{2 / 09 / 90}$

8 - Deliver wo information briefing, Version $1-\underline{2 / 16 / 90}$

9 - Deliver a draft of "A review of RCRA land disposal restrictions as related to the U.S. Department of Energy's Environmental Restoration Program" $\underline{2 / 20 / 90}$

10. Deliver the EM-40 information briefing, Version $2-\underline{2 / 23 / 90}$

11 - Draft EM Management Plan Information - $3 / 27 / 90$

12 - Deliver final draft of wo briefings with photos to DOE for review - $4 / 11 / 90$

13 - Deliver first draft of EM briefings to Technology Development for review $\underline{4 / 12 / 90}$

14 - Deliver final draft of ER briefings to DOE for review - $4 / 20 / 90$ 
15 - Review of RCRA land disposal restrictions as applied to the U.S. Department of Energy's Environmental Restoration Program (second Draft) - 4/27/90

16 - Meeting at DOE Headquarters to discuss EH and HAZWRAP comments to study of $L D R^{\prime} s-\underline{5 / 2 / 90}$

17 - Incorporate EH and HAZWRAP comments. Sent updated study of LDR's to the field - $\underline{5 / 11 / 90}$

18 - Review of RCRA land disposal restrictions as applied to the U.S. Department of Energy's Environmental Restoration Program (Final Document) - $5 / 18 / 90$

19 - Deliver wO briefings to DOE for final review - $5 / 23 / 90$

20 - Deliver ER briefings to DOE for final review - $5 / 23 / 90$

21 - Deliver for review wo \& Er briefs for Australian visit - $\underline{5 / 30 / 90}$

22 - Deliver ER \& WO Australian briefs - $\underline{6} / 6 / 90$

23 - Deliver ER \& WO briefs to DOE for production - $6 / 11 / 90$

24 - Complete participation on FY 92-96 Five Year Plan Update - 6/25/90

25 - Deliver to DOE Headquarters the first draft of the EM booklet - $7 / 11 / 90$

26 - Meet with DOE Headquarters to recelve comments on the EM booklet - $7 / 20 / 90$

27 - Deliver the EM-2 EM Booklet to RIch Aiken with headquarters' comments resolved - $8 / 13 / 90$

28 - Deliver the EM-2 EM Booklet to Paul Grimm for final review - $8 / 31 / 90$

\section{INFORMATION SYSTEMS/LONG-TERY (301)}

11 - Deliver DWTM/ISS project overview - 10/12/89

12 - Deliver data input forms for waste tracking - 12/23/89

\section{MANAGEMENT (901)}

3 - Deliver second-year program plan - $12 / 30 / 89$

4 - Deliver annual performance report for 10/89 - $9 / 90$ : "Research and Development of Methods and Tools for Achieving and Maintaining Consensus Processes in the Face of Change Within and Among Government Oversight Agencies" - $6 / 2 / 90$ 


\section{THIRD YEAR STUDY RESULTS: KEY TO FIGURE 11}

CONSENSUS PROCESS/SHORT-TERY (250) $\cdot(260)$

29 - Deliver a draft of the DT\&E Guidance Documents to (Office of Technology Development) EM-55 for review - 10/01/90

30 - Deliver first draft of the Morgantown Energy Technology Center (METC) briefing to Michael Barainca - 10/19/90

31 - Send the final draft of the EM booklet to Rich Alken for final review by Leo Duffy and the Secretary - 10/22/90

32 - Deliver METC briefing to Michael Barainca with his comments incorporated $\underline{10 / 26 / 90}$

33 - Deliver final METC briefing to Michael Barainca - $11 / 1 / 90$

34 - Approval received on DOE site map and EM booklet is delivered to DOE Public Affairs for production - $11 / 19 / 90$

35 - Print EM booklet distributed to DOE-HQ and MSL from DOE Public Affairs $1 / 14 / 91$

36 - Distribute guide entitled "A Review of RCRA Land Disposal Restrictions as applied to the U.S. Department of Energy's Environmental Restoration Program" to the field and to DOE-HQ - 1/14/91

37 - Deliver to EM-55 an independent assessment of ISV technology program development activities - $2 / 7 / 91$

38 - Deliver the first draft of new fact sheets describing DOE's Environmental Restoration and Waste Management for internal use and for communication with the general public - $2 / 25 / 91$

39 - Attend Stakeholders' meeting in Arlington and compile comments of the breakout groups into issues to be addressed by Duffy and within the FiveYear Planning Process - 5/20/91

40 - Meet with Anne Bisconti of the U.S. Council on Energy Awareness to learn about its public opinion survey methods for commercial nuclear organizations - $9 / 16 / 91$

\section{INFORMATION SYSTEMS/LONG-TERY (301)}

13 - Meet with (Waste Operations) EM-35 to discuss regulatory Compliance Tracking and reporting Systems Survey - 3/6/91

14 - Deliver survey draft scoping agreement to EM-35 .. $3 / 15 / 91$

15 - Deliver survey final scoping agreement to EM-35 - $\underline{3 / 22 / 91}$ 
INFORMATION SYSTEMS/SHORT-TERY $(360),(530),(540),(610),(710)$

16 - Sent letter describing study to field offices - 4/1/91

17 - Recelve list of contacts from field offices - 4/15/91

18 - Call field office contacts to schedule visit - $4 / 30 / 91$

19 - Complete field office visits - $6 / 28 / 91$

20 - Complete initial data assembly from field office visits - $7 / 31 / 91$

21 - Complete clarification phone calls with fleld office contacts - $8 / 23 / 91$

22 - Complete draft survey results and send to field office contacts - $\underline{8 / 30 / 91}$

23 - Receive comments on draft survey results from field office contacts $\underline{9 / 30 / 91}$

\section{MANAGEMENT (901)}

5 - Deliver annual performance report for 10/90 - 9/91: "Research and Development of Methods and Tools for Achieving and Maintaining Consensus Processes in the Face of Change Within and Among Government Oversight Agencies" - 3/29/91 
FOURTH YEAR STUDY RESULTS: KEY TO FIGURE 12

CONSENSUS PROCESS/SHORT -TERY (290), (510), (640), (670),680)

41 - Review EM-30 preliminary configuration and deliver options for how we might be useful to Coleman \& Cowan - 10/10/91

42 - Test EM booklet on EM interns - 10/24/91

43 - Test fact sheets on MSL students - 11/11/91

44 - Develop survey for and attend the Hazardous Materials Transportation Workshop - 11/13/91

45 - Give presentation to Cowan \& Gerstein on our idea of what we'd do with the Configuration Study and how it was tied to the PEIS - $11 / 25 / 91$

46 - Meet with Steve Cowan to discuss survey of members of the public who submitted questions to DOE - 11/26/91

47 - Analyze draft PEIS commitments and deliver to Bob Morgan and Gerstein $12 / 10 / 91$

48 - Write background draft research report on risk research for Morgan, PEIS $12 / 15 / 91$

49 - Write Configuration Study overview and deliver to Gerstein - 12/20/91

50 - Write scope document for the Configuration Panel and deliver to Gerstein $1 / 10 / 92$

51 - Attend DOE Public Participation Workshop and National Science Foundation Public Hearing to learn more about DOE audiences - 1/14/92

52 - Attend the Waste Operations Resource Allocation Support System National Workshop to evaluate the review group's effectiveness in providing input to meet WO's Support System development needs - 1/29/92

53 - Form an academic panel for Configuration Study and summarize the first initial questions for Gerstein - 2/18/92

INEORMATION SYSTEMS/SHORT-TERY $(360),(530),(540), 610), 710)$

24 - Complete data investigation and collection - 10/18/91

25 - Complete initial draft of Hazardous Report - 11/8/91

26 - Complete final draft of Hazardous Report - 12/2/91

27 - Receive and collect all outstanding data - 1/30/92

28 - Complete initial draft of Sanitary Waste Background Report - $2 / 21 / 92$ 
29 - Complete final draft of Hazardous Waste Background Report - $3 / 6 / 92$

30 - Provide computer text and graphics files to EM-351 for final Hazardous Waste Background Report - $\underline{3 / 10 / 92}$

31 - Receive comments on initial draft of Sanitary Waste Background Report $\underline{3 / 11 / 92}$

32 - Complete final draft of Sanitary Waste Background Report - $\underline{3 / 20 / 92}$

33 - Provide computer text and graphic files to EM-351 for final Sanitary Waste Background report - $\underline{3 / 24 / 92}$

\section{MANAGEMENT (901)}

6 - Deliver annual performance report for 10/91 - 9/92: "Research and Development of Methods and Tools for Achieving and Maintaining Consensus Processes in the Face of Change Within and Among Government Oversight Agencies" - $3 / 30 / 92$ 
RESOURCES AND GRANT MANAGEMENT

SUMMARY

Accomplishing the proposed research objectives requires a five-year project period at a total cost of $\$ 8,933,193$. MSL is experienced at conducting large research efforts and will apply proven management techniques to this significant effort. The budget reflects the considerable commitment from the faculty, staff, and students at MSL and from contributing faculty.

The Principal Investigator and MSL Research Faculty maintain a reasonably constant contribution throughout the grant period. To satisfy the criteria of the technical approach, we need to purchase, install, and operate research equipment.

\section{GRANT MANAGEMENT STRUCTURE}

As previously discussed, the research efforts are divided into three domains (fundamental research, consensus process studies, and information systems studies). We define studies within each domain. Each study is assigned a DOE study manager for technical direction and an MSL study manager. The quarterly report reviews the scope, status, milestones, and budget for each study. A copy of all quarterly reports and grant deliverables is provided to the DOE Consensus Grant technical manager.

A DOE Consensus Grant Steering Committee makes sure the academic results of the grant are achieved. The DOE Consensus Grant Steering Committee members, the DOE Consensus Grant technical manager, and the MSL Consensus Grant manager meet each quarter. This quarterly review focuses on evaluating the overall grant academic progress and is structured to review and evaluate our progress in meeting the grant and topical area objectives. The DOE Consensus Grant technical manager maintains copies of the quarterly reports. The topical areas are evaluated for applicability, appropriateness to the research efforts, and overall priority.

MSL tracks and allocates resources (personnel and budget) both at the "study" level and one level deeper, the "task" level. Personnel and budget data from our internal accounting system are provided to the DOE study managers (who control their own budgets) and the DOE Consensus Grant Steering Committee members (who control allocation of funds to studies). This information is used to control and focus the research efforts. 
EQUIPMENT

First Year

Because of the fortuitous events, we focused on test sites and consensus groups during the first grant year. Therefore, we delayed our design and procurement for the information gathering and communications laboratories. We spent very little money because we rearranged our priorities to take advantage of a target opportunity.

Second Year

As in the first grant year, opportunities for c'serving and participating in consensus groups during the second grant year resulted in spending less equipment dollars than we anticipated. We expect to begin organizing our communications laboratory before the end of the second grant year.

Third Year

During the second grant year, we had unanticipated heavy personnel and travel expenditures because of our work on the Five-Year Plan. We delayed buying equipment until the third year of the grant. The first half of year three was spent scoping out our equipment needs.

Fourth Year

We continue to adjust our equipment expenditures as the fiscal demands of personnel and travel continue to be greater than expected. We have completed our equipment acquisition plan and continue to maintain it with respect to the latest in avallable technology. Several items have been procured and have proven valuable in our data collection efforts. We'll initiate the purchase of the remaining critical components necessary to support the conceptual modelling of EM in year four as funds permit.

\section{FINANCIAL}

\section{First Year}

One of the outcomes of the fortuitous events we keep referring to is the Five-Year Waste Plan. Due mostly to that effort and less to efforts on the presidential transition and networking, we spent the funds on our budget line items differently than planned. We don't believe we can yet evaluate the full extent the impact of the Five-Year Waste Plan will have on our budgeting.

We overspent personnel and travel and underspent equipment as well as the UME subcontract. This change didn't lessen the importance of equipment and the UME subcontract, but reflected the opportunity of the culture change toward consensus. Next year and possibly in future 
years, we expect the reverse shift from the budget in our proposal. For example, we plan to implement our equipment acquisition next year.

Second Year

The second year budget includes the proposed amount and the carryover from the first year. The focus on consensus and the Five-Year Waste Plan has created demands beyond what was budgeted for personnel and trave1. If demands continue for several months, we may need to request some equipment dollars be liquidated to cover increased costs on these budget line 1 tems.

Third Year

The third year budget includes the proposed amount and the carryover from year two. We requested and recelved permission to liquidate equipment funds planned for the second grant year to cover heavy personnel and travel expenditures incurred during our work on the FiveYear Plan.

During the third year, we submitted to DOE our reques't to reallocate funds for the remainder of the third grant year to purchase equipment.

Fourth Year

We didn't receive the total funds proposed for year four. In addition, the unanticipated demands of personnel and travel in year three resulted in a slightly negative carryover. As a result, we've had to reduce some of our study efforts and prematurely close out several studies. 


\section{Graduate Students Performing Consensus Grant Related Research for Advanced Degrees}

Student

D.M. Brubaker

B.H.

Glickstein

D. Mercer

E. O'Sullivan

J. W. Polk

E. V. Shrock

R. Fulmer

M. Grunau

J. F. Keeling

J. Moore

J. L. Peterson

C.M. Walsh
Degree

MS

MS

MS

MS

MS

MS

MS

MS

MS

PhD

MS

MS
Effort Area

Consensus Tools for Methods

Theory and

Concepts

Theory and

Concepts

Consensus Tools

for Rules

Consensus Tools

for Rules

Consensus Tools

for Rules

Consensus Tools

for Methods

Consensus Tools

for Rules

Consensus Tools

for Methods

Consensus Tools

for Methods

Test Sites

Consensus Tools

for Rules

\section{Grant-yr \\ started}

1

1

1

1

1

1

1

2

2

2

2

3
Status

finished

July 1991

changed

degrees

changed

degrees

changed

degrees

finished

July 1991

changed

degrees

finishing

December 1992

finished

December 1991

finished

December 1991

changed

grants

finished June 1991

finishing June 1993 

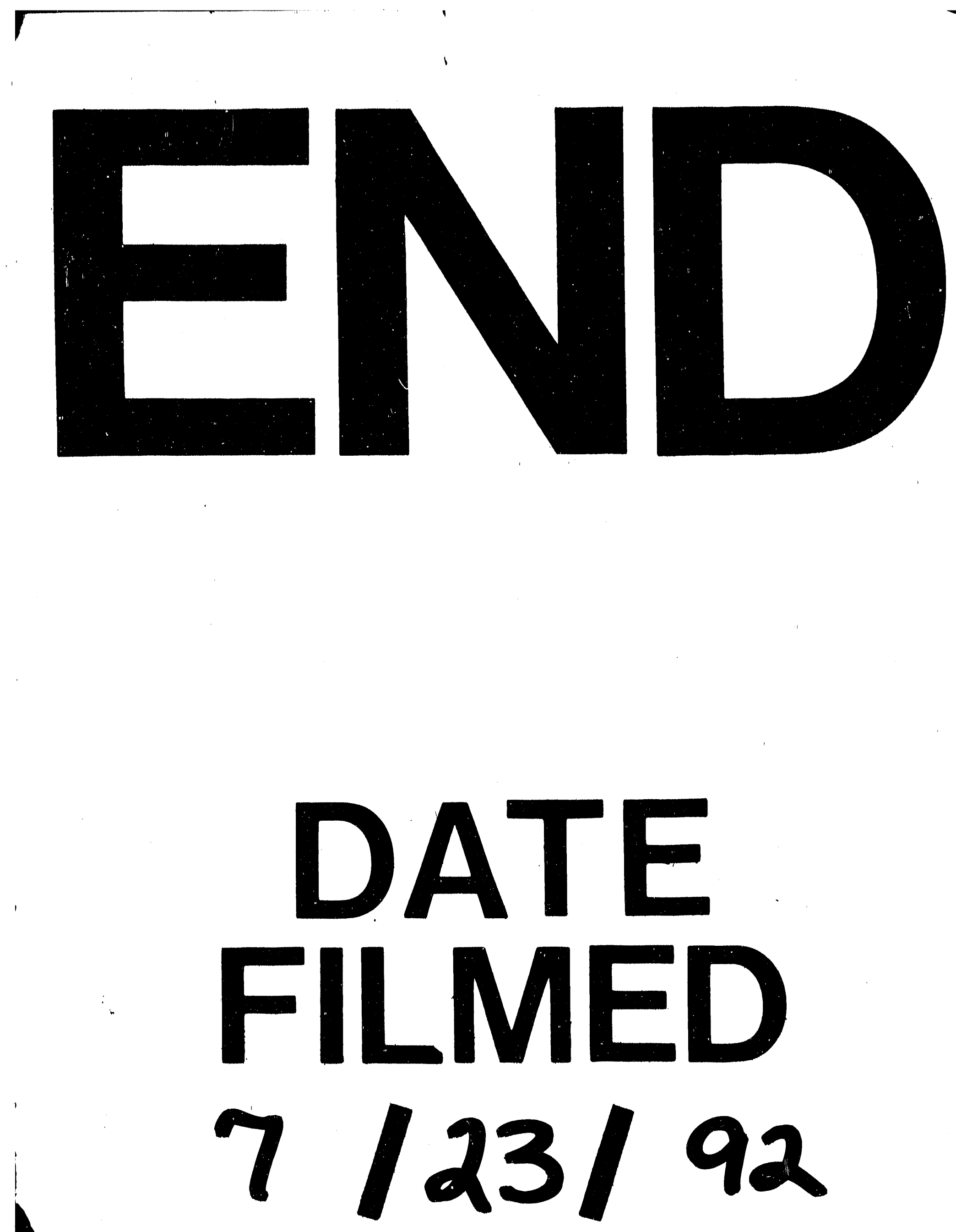
. 